Portland State University

PDXScholar

5-6-1996

\title{
Understanding Pre-service Teachers' Conceptualizations of Diversity
}

Terrine Louise Borwn Vogt

Portland State University

Follow this and additional works at: https://pdxscholar.library.pdx.edu/open_access_etds

Part of the Speech and Rhetorical Studies Commons

Let us know how access to this document benefits you.

\section{Recommended Citation}

Vogt, Terrine Louise Borwn, "Understanding Pre-service Teachers' Conceptualizations of Diversity" (1996). Dissertations and Theses. Paper 5146.

https://doi.org/10.15760/etd.7022

This Thesis is brought to you for free and open access. It has been accepted for inclusion in Dissertations and Theses by an authorized administrator of PDXScholar. Please contact us if we can make this document more accessible: pdxscholar@pdx.edu. 
THESIS APPROVAL

The abstract and thesis of Terrine Louise Brown Vogt for the Master of Science in Speech Communication were presented May 6, 1996, and accepted by the thesis committee and the department.

COMMITTEE APPROVALS:
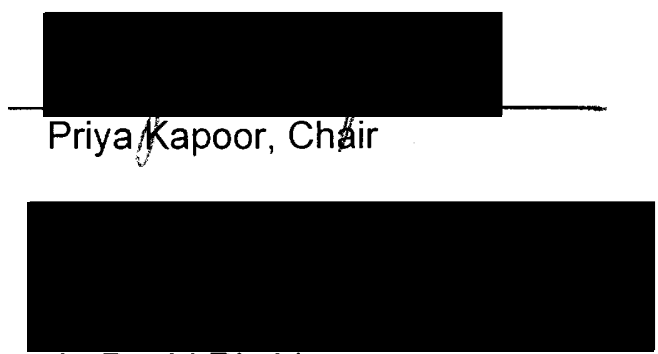

L. David Ritchie

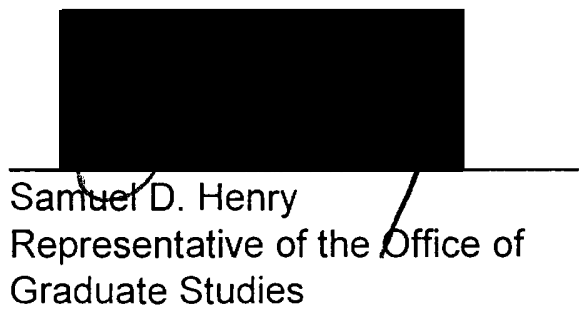

DEPARTMENT APPROVAL:

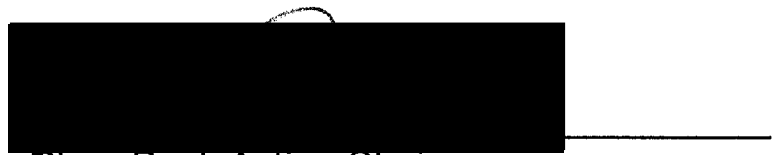

Rhea Paul, Acting Chair

Department of Speech Communication 


\section{ABSTRACT}

An abstract of the thesis of Terrine Louise Brown Vogt for the Master of

Science in Speech Communication presented May 6, 1996.

Title: Understanding Pre-service Teachers' Conceptualizations of Diversity

"Diversity" has become a word associated with many professions, institutions, and contexts, paired with words such as standards, consultants, training, awareness and others. However, the researcher focused on how diversity is defined or conceptualized by pre-service teachers at Portland State University. Diversity is especially important as it relates to educators and pre-service teachers. Teachers will, today, be instructing children of different backgrounds and races more than ever in the past (Ross \& Smith, 1992). And teachers conceptualizations and behaviors have profound impacts on student performance and success.

In an effort to come to understand the ways pre-service teachers conceptualize "diversity," a literature search was conducted to identify past and present conceptualizations of diversity within the institution of education and more broadly. In addition, an open-ended survey question was administered, and two focused group discussions and three in-depth 
interviews were conducted. The survey question and transcripts from the focus groups and interviews were analyzed in an effort to identify emergent themes or units of meaning.

Two major theoretical assumptions were utilized: lower-order concepts and constructivism. First, Chaffee's (1991) lower-order concepts, smaller units which comprise a larger unit of meaning, the higher-order concept, was selected. The education literature identifies language, learning style, teaching style, race, socio-economic status, ability, gender, and others (see APPENDIX B) as lower-order concepts for the higher-order concept "diversity." Second, according to constructivism, the human actively interprets and makes meaning of events (Delia, 1977) using constructs, pairs of bi-polar opposites.

Individuals identify an event, experience or object and classify it along a number of relevant constructs in order to make sense of it in relation to similar phenomena. Within the education literature, the constructs, assimilationism / pluralism and ethnocentrism / ethnorelativism, good / bad, growing / dying and others have clearly been utilized to make meaning of "diversity."

The researcher identified themes respondents appeared to correlate with the concept "diversity" and many complicated and conflicting messages as well (not unlike the "diversity" literature). Two of the emergent themes suggest additional lower-order concepts: "Structures" and "Mixed Messages." The emergent themes "Teachable" and "Uncertain" suggest new constructs. 
UNDERSTANDING PRE-SERVICE TEACHERS' CONCEPTUALIZATIONS

OF DIVERSITY

by

TERRINE LOUISE BROWN VOGT

A thesis submitted in partial fulfillment of the requirements for the degree of

\author{
MASTER OF SCIENCE \\ in \\ SPEECH COMMUNICATION
}

Portland State University

1996 


\section{ACKNOWLEDGMENTS}

I would like to acknowledge the assistance and insights contributed by

Dr. Priya Kapoor, Dr. L. David Ritchie and Dr. Samuel D. Henry without whose support and direction this work would be less than it is. I wish to express my gratitude for the many hours of generous counsel they provided.

Special thanks to the members of the Children and Communities Cohort for allowing me to be a part of their educational experience and for providing me with a tremendous education in the process. Without their generosity this inquiry would not be possible.

Special thanks also to my spouse, Johnathan, and my parents, Terry and Dolly, and family for patience with my "big project" and for sharing ideas. Additionally, I would like to thank the graduate teaching assistants in the PSU Speech Communication Department, and especially my office-mate Shawn Riffel, for their constant support and the "gift of gab." 


\section{TABLE OF CONTENTS}

\section{CHAPTER}

1 THEORY

1

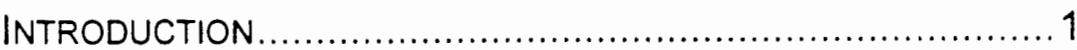

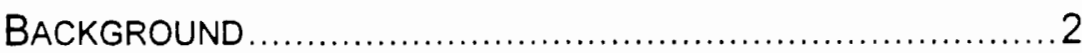

HISTORICAL DEFINITIONS OF "DIVERSITY" IN OTHER FIELDS ..6

HISTORICAL CONCEPTUALIzATIONS OF "DIVERSity" IN

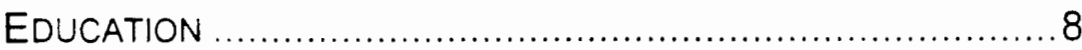

CURRENT CONCEPTUALIZATIONS..................................... 11

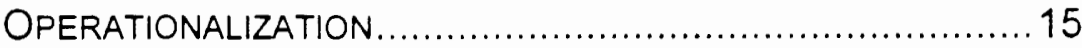

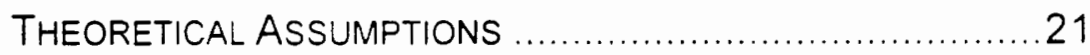

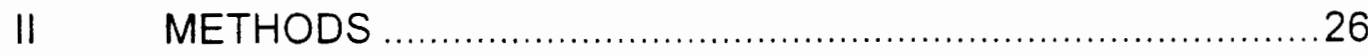

General Methodological Considerations.................26

METHODOLOGICAL PARTNERSHIP ................................... 32

RESPONDENTS .......................................................... 33

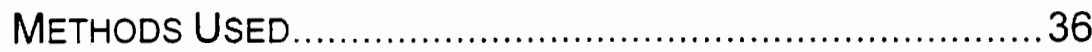

Literature Review

Survey

Focus Group 
Pilot Study

In-Depth Intervielws

III RESULTS

FOCUS GROUPS. 69

Structures

Types Of Diversity Or Criteria

Mixed Messages

Teachable

IN-DEPTH INTERVIEWS. .83

Structures

Types Of Diversity Or Criteria

Mixed Messages

Teachable

IV DISCUSSION

GENERAL FINDINGS.

Structures

Types Of Diversity Or Criteria

Mixed Messages

Teachable

Uncertain

General Suggestions 120

SUgGESTIONS For FUtURE RESEARCH

APPENDIX

A 


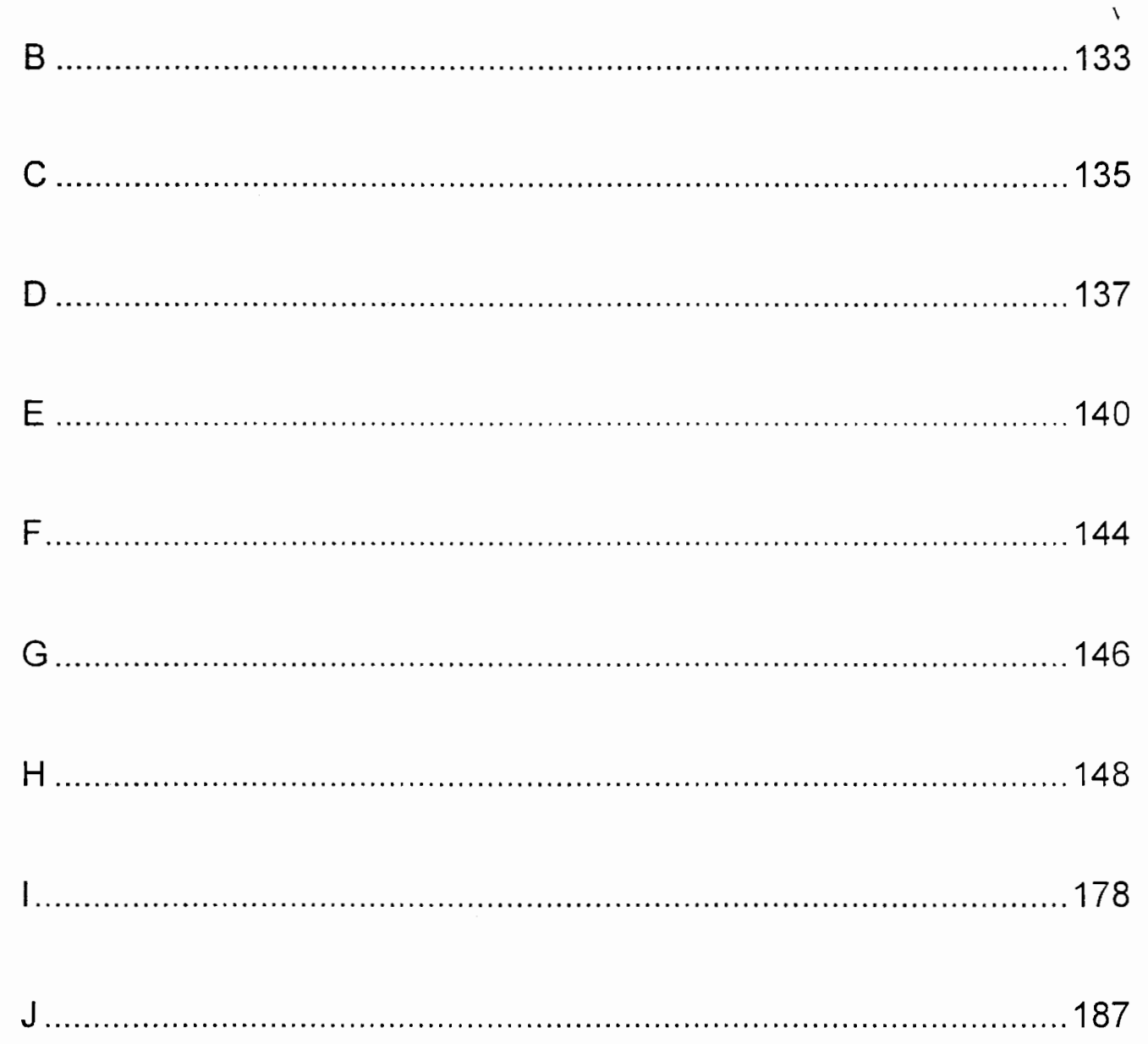




\section{CHAPTER I}

\section{THEORY}

Introduction

Diversity training, diversity consultants, diversity requirements. diversity focused, diversity sensitive... "diversity" is a large part of today's vocabulary. Because of the newly developing trend toward diversity related services and requirements, especially in the field of education, it has become necessary to reevaluate the meaning of the word "diversity."

"Diversity" and "multiculturalism" are concepts eternally defined and discussed but rarely researched in the field of education. Currently, and for many years, a debate has raged regarding the purpose of education with positions ranging from a stance that education should be in some manner emancipatory (Green, 1978) to a view of education designed to prepare individuals for the workplace, to a belief in education developing the potential of the individual (Carnoy \& Levin, 1985). Within the middle of this debate stand the concepts "diversity" and "multiculturalism." It is hotly contested where these concepts fall in relation to the purpose of education, generally. While some argue "multiculturalism" and "diversity" are useful only as they 
assist in instilling one set of values and beliefs, the "melting pot" metaphor, others assert the value of maintaining and respecting differences. Still others view diversity as a characteristic of people, or a teaching style or a desired end state. Certainly, the discussion is complicated, many faceted and often times contradictory. The importance of this debate is only increased and highlighted by research that has shown teachers' conceptualizations of students have significant effects on student performance (Ladson-Billings, 1994). However, despite this rhetoric, little has been done to uncover the ways "diversity" or "multiculturalism" is currently conceptualized within the institution of education, at the level of the teacher or instructor. While many position papers exist that indicate the meaning of diversity to education officials at some higher, remote level, teachers themselves are seldom probed for their input.

\section{Background}

The author has chosen to examine "diversity" in this context, as opposed to its partner "multiculturalism," because of the more common use of the word "diversity." After an informal, preliminary inquiry, conducted (by the author) with students in a university environment, it was determined most individuals have had little exposure to the term "multiculturalism." When asked to articulate their understanding of "multiculturalism," few students were 
able to even make an attempt. As a result, "diversity," a more commonly used term, was selected for its salience.

However, the author believes the selection of "diversity," as opposed to "multiculturalism," to be a minor limitation for a number of reasons. Most importantly, in the education literature these terms are used synonymously. One often encounters "diversity" and "multiculturalism" used interchangeably within one publication: "diversity training," "multicultural training," "valuing diversity," "valuing multiculturalism." "diverse population," "multicultural population." Additionally, when these concepts are defined, they are most often explicated in terms of one another. James A. Banks, Director for the Center for Multicultural Education at the University of Washington, defines "multicultural education" as follows:

Multicultural education tries to create equal educational opportunities for all students by ensuring that the total school environment reflects the diversity of groups in classrooms, schools and the society as a whole...Multicultural education has emerged as a vehicle for including diverse groups and transforming the nation's educational institutions. (Banks, 1994, p. 4)

Nieto, in her 1992 book Affirming Diversity, states multicultural education is education that "affirms diversity," that takes "into account the diversity of our student body." (p. 222). Similarly, multiculturalism itself has been defined in terms of diversity:

Multiculturalism, this group believes, should not only develop appreciation for the perspectives of others, but should also sustain a value-tolerant acceptance of diverse cultural 
understandings, belief systems, customs, and (perhaps)

sociopolitical traditions. (Janzen, 1994, p. 9)

Finally, the history of these two terms in the education electronic data base, ERIC, follows a similar path, including much cross referencing. Both items begin to appear regularly about 1973 and continue to the present with some early preference for "diversity" and current preference for "multiculturalism."

Determining educators' conceptualizations of the term "diversity" is a necessary and valuable task for a number of reasons including changing demographics and the so called Pygmalion Effect. Pygmalion Effects are effects on student performance resulting from teachers' opinions regarding students' abilities.

The changing demographic trends in the United States have been well documented and lead to the conclusion that most people in this country will experience increased interaction with individuals from other cultures as time progresses. According to the 1989 Census Bureau projection, during the next four decades (1990-2030), the white population of the United States will grow by about 25 percent, the African-American population by 68 percent, the Asian-American, Pacific Island-American, and American Indian populations by 79 percent, and the Latino or Hispanic population of the United States by 187 percent (Cortes, 1991). This leads to direct implications in America's classrooms. Teachers will be teaching more students unlike themselves in terms of race, culture and background, "... a result of demographic shifts of the 
1980 's today's teachers are far more likely than their predecessors to teach children with backgrounds different from their own." (Ross \& Smith, 1992, p. 94). Additionally, current statistics regarding teachers of color in the American school system indicate teachers of color are highly underrepresented:

For example, whereas the percentage of African American students in our public schools is 16.2 percent, only 6.9 percent of the teachers are African American...At present, 91 percent of our teaching force is White and English-Speaking. This percentage is expected to increase even more. (Nieto, 1992, p. 6)

Because of this increased direct and influential exposure to individuals different from themselves, it is important to know the ways teachers understand diversity.

A major implication of educators' conceptualization of "diversity" can be explained in terms of Pygmalion Effects. These effects constitute student behavior that is a result of teachers' preconceived expectations. Simply put, "...how teachers...think about education and students makes a pronounced difference in student performance and achievement." (Ladson-Billings, 1994, p. 22). This phenomenon has been likened to the self-fulfilling prophecy or the idea that "... when we expect certain behavior of others, we are likely to act in ways that make the expected behavior more likely to occur" (Rosenthal \& Babad, 1985, p. 36). Teachers often have preconceived expectations for students. In these cases, teachers may take action to bring about those expectations, resulting in student behavior that fulfills teachers' 
preconceptions. Historically, teachers have been found to treat students randomly labeled as "gifted" differently than those randomly labeled "nongifted" (Rosenthal \& Jacobson, 1968; Rubovits \& Maehr, 1973; Rubovits \& Maehr, 1971). Additionally, research suggests black students have been treated differently than white students based upon similar preconceived teacher expectations:

Black students were given less attention, ignored more, praised less and criticized more...it cannot be argued that teachers were responding to any actual intellectual differences between black students and white students or to any incongruity between label and actual potential. (Rubovits \& Maehr, 1973, p. 217)

Current research demonstrates no change in this disturbing trend (King \& Ladson-Billings, 1990). The prospect of increased exposure to cultural variation in the classroom only serves to highlight the problems that can occur as a result of negative preconceptions about students.

\section{Historical Definitions Of "Diversity" In Other Fields}

The concept "diversity," as it is understood in education, developed within the context of other definitions of the concept. One such definition is the biological conceptualization of "diversity," perhaps one of the earliest influences. It appears the concept "diversity" developed closely with the concepts of variation and evolution in the biological sciences. To explain and describe the variety of biological organisms present on the earth and to 
explore the variation of life, both human and otherwise, the concept "diversity" was employed. The term "biological diversity" is commonly encountered in even the earliest texts to address evolution. The concept is used to describe differences present in biological organisms as a result of evolutionary development, "Darwin developed the doctrine of natural selection as a single principle capable of explaining the origin of species, biological diversity, and similarities among related life forms." (Kottak, 1982, p. 27).

"Diversity" is also employed to describe the physical differences between groups of humans just as it describes the physical differences between other organisms (Kottak, 1982). In fact, a book entitled The Diversity of Man, by Robin Clarke (1964) is devoted, in part, to addressing the evolutionary development of physical variation among humans, including skin color, hair characteristics, body types, facial structure etc.

The anthropological studies have long used "diversity" to describe both the physical, biological differences and cultural differences between humans (UNESCO, 1955). In addition to addressing the physical variation in The Diversity of Man, Clarke explores differences in human culture, "In short, man's culture is the product not only of his individuality but also of his society in a particular place at a particular time. Physically man varies in much the same way." (1964. pp. 9-10). According to Carrithers (1992) well established before the Second World War was the search for diversity defined as "specific 
knowledge of hitherto uncontemplated versions of social life." (p. 12).

Therefore, the anthropological studies included and emphasized the addition of human cultural differences to the concept "diversity," a development which most certainly influenced the concept as it is understood in education today.

Historical Conceptualizations Of "Diversity" In Education

Since the colonization of the United States, the concept "diversity" has been a major part of the formal education system. As early as the 18th and 19th centuries there were bilingual education programs designed to address the "language differences" dimension of diversity. Programs were established in German - English, Czech - English, Italian - English, Polish - English, Norwegian - English, Dutch - English and Cherokee - English (Worsnop, 1993). However, with the rise of nationalism and the immigration restrictions that accompanied World War I, these programs were all but wiped out. Bilingual education began its resurgence in 1959 when Fidel Castro came to power in Cuba. Cuban refugees, mostly of European descent, streamed into Dade county Florida. And in 1963 a bilingual program was established to assist their children in adapting to the new environment. The program's goal was to "foster bilingualism among native speakers of both tongues" (p. 707). Legislation later became an impetus for instituting bilingual education programs. In the 1974 supreme court case, Lau v. Nichols, the court ruled 
"...unanimously that the civil rights of students who did not understand the language of instruction were indeed being violated." (Nieto, 1992, p. 162). The court stated, "There is no equality of treatment merely by providing students with the same facilities, textbooks, teachers, and curriculum; for students who do not understand English are effectively foreclosed from any meaningful education. "(Lau v. Nichols, 1974. 414 U.S. 563). This set the tone and legal president for seekers of bilingual programs; in fact, bilingual education had become a legal right.

"Diversity" has also been understood in relation to the concept "democracy." Since the inception of American education, democracy, as a theme, has been at the forefront of educators' considerations. Individuals utilizing the American education system are expected to learn to contribute to a democratic society and behave as responsible, informed citizens:

What is the true end of American education?... we cannot make the United States a democracy without education. The true end of American education is the knowledge and practice of democracy - whatever other personal ends an education may serve. (Sharp, 1922, p. 36)

Therefore one of the primary functions of education has been established as preparing individuals to function in a democratic society. Part of the democratic ideal, however, are the concepts of equality, equal participation and the welfare of the whole as stated by Dallas Sharp (1922) in Education in a Democracy: 
"...natives and foreigners mingling, and where better than in the same school? - the foreigner changing by the experience, the native changing by the same experience, many necessary things of fact and spirit about his own duty as a citizen" (p. 18).

According to this early conception of democracy, citizens must mingle and participate together, fully, in the democratic process, "If we go farther South, we shall find another segregation of white and black children in the schools, both being educated for life and living but neither for living together, for democracy." (Sharp, 1922, p. 67). Thus, unity under democracy is clearly stated in terms of race and origin. Generally, a democratic society should care for the welfare of all its citizens. In his 1924 book Education for Citizenship, Almack states the life of a citizen must at least include "Experiences in cooperative activities directed toward the achievement of a common purpose and for the common good." (p. 3).

In addition to the early "language difference" and "democratic" conceptualizations of "diversity," learning style differences and pedagogical differences have been a consideration in education since the 1960's. A result of psychological research, these concepts had a large impact on the education community. Currently, programs designed to promote diverse teaching styles, and accommodate diverse learning styles, exist in teacher education curriculum across the country. In the Los Angeles Unified School District, teachers are trained to consider diverse learning styles and pedagogical approaches by attempting to bring to consciousness awareness 
that "...tasks represent subject matter in distinctly different ways and have the potential for distinctly different learning consequences." (Williamson McDiarmid, 1992, p. 88). Finally, opinion papers regarding the need for consideration of different learning and teaching styles in the classroom are numerous, "Cultures do have distinctive learning style patterns, but the great variation among individuals within groups means that educators must use diverse teaching strategies with all students." (Guild, 1994, p. 16).

Despite the fact desegregation had been an issue in education for at least a decade, it is the change of atmosphere that occurred in the 1960's that brought a new consideration of cultural differences in the education literature. It is not until this time that one encounters "cultural diversity" in mainstream education literature. While its inceptions are hazy, this category acts as a collection of leftovers and appears to encompass race, gender, socioeconomic status, ability, and currently, sexual orientation.

\section{Current Conceptualizations}

The education literature is riddled with opinion papers regarding the debate over the meaning of the concept "diversity," and its relation to "multiculturalism," and the implications this definition might have for the education system (Banks, 1994; Elrich, 1994; Guild, 1994; Janzen, 1994). Many educators approach the concept from the angle of valuation of human 
differences. In this area, generally, two opinions exist regarding "diversity" or "multiculturalism" and its functions:

Indeed, two different - and diametrically opposed - perspectives of multiculturalism are currently vying for predominance. The goal of cultural pluralism is that ethnic groups will remain intact and that their idiosyncratic ways of knowing and acting will be respected and continued. Assimilationism, on the other hand, accepts the importance of understanding multiple beliefs, but has as its primary goal the amalgamation of all groups into the American mainstream. (Janzen, 1994, p. 9)

These two perspectives implicate nearly opposing concepts of "diversity" or multiculturalism (Banks, 1994; Guild, 1994). The culturally plural approach suggests a definition comprised of valued cultural differences to be maintained while the assimilationist approach indicates unvalued differences designed to be minimized.

In addition to these conceptualizations of "diversity," there are some educators who understand "diversity" in terms of learning styles and varying pedagogical approaches. Sonia Nieto, in her book Affirming Diversity (1992), suggests teachers are products of educational systems that have histories of racism, and unaccommodating pedagogy. As a result of their experiences with the educational system, teachers put into practice what they themselves have encountered and perpetuate structures that may be harmful to many of their students. She highlights the opinion of educators by stating learning styles and pedagogical variation are crucial to the concept "diversity":

... our understanding of the learning process is the basis for decisions about curriculum and instruction. They are important 
because success for the diverse populations that schools serve calls for continual reexamination of educators' assumptions, expectations, and biases. (Guild, 1994, p. 16)

Additionally, the learning style and pedagogical variety focus is widely accepted and has become a part of the National Council for the Social Studies Curriculum Guidelines for Multicultural Education. Consideration of diverse learning styles is in the top five of twenty-three issues, "5.0 The curriculum should reflect the cultural learning styles and characteristics of the students within the school community." (Banks, 1992. p. 281).

Recently, in an article titled "Recognizing Diversity" Cheng addressed the language differences dimension of "diversity," "...a paradigm shift has been proposed in an attempt to restructure the present curriculum by encompassing cultural and linguistic characteristics of minorities". (Cheng 1990). This "language differences" definition of "diversity" represents one of the oldest and most discussed in the education field (Worsnop, 1993). Bilingual education programs appeared in the United States before the 18th and 19th centuries and included languages such as German, Czech, Italian, Polish, Dutch, and Cherokee. While these programs went through a period of decline during the World War I period with the resurgence of nationalism, they were revived in the 1960's and continue to the present. According to Banks, Director of the Center for Multicultural Education at the University of Washington, "A multicultural curriculum recognizes language diversity and 
promotes the attitude that all languages and dialects are valid communicating systems for some groups and for some purposes." (Banks, 1992. p. 287). Additionally, guideline number twenty in the Curriculum Guidelines for Multicultural Education prepared by the National Council for The Social Studies states "The multicultural curriculum should provide opportunities for students to study ethnic group languages as legitimate communication systems and help them develop full literacy in at least two languages." ( $p$. 287).

In addition to these well articulated conceptualizations of diversity in the education literature, there are others which remain hazy and unclear. Often multicultural education is required to "...reflect ethnic, cultural, and gender diversity" (Banks, 1992. p. 274) in one sentence (including ethnicity, culture and gender in the concept) and required to "...reflect the racial, ethnic, and cultural diversity" (p. 274) in another (redefining the concept to include race but not gender). Yet in most cases definitions of diversity are not operationalized to the level of indicating who is included in the concept and who is not. What groups of people constitute these categories of "diversity?" The literature alone is not sufficient to provide a clear understanding of the ways educators conceptualize "diversity." This last, hazy, category or dimension of diversity in the education literature is labeled "cultural diversity" 
and appears to encompass age, gender, race, ethnicity, cultural background, ability, and sexual orientation.

\section{Operationalization}

The concept "diversity", or the ways people understand the concept "diversity," has been operationalized, or measured, in several ways (Bennett, 1986; Lieberman, 1994; Paine, 1990). However, few of these operational schemes have been used in empirical research to date, providing little in terms of reliability for any of these measurements. Additionally, nearly all measurements to date rely on one singular dimension of the concept "diversity." Yet, as has been demonstrated, the concept has many dimensions to be explored.

One measurement system, however, stands apart for its exemplary operational definitions: Bennett (1986) "A Developmental Approach to Training for Intercultural Sensitivity". Bennett's work is presented as a strict continuum of development from ethnocentrism to ethnorelativism which may represent its primary weakness and limiting factor. However, if one considers his work as a simple taxonomy it becomes quite useful for providing a descriptive picture of an ethnocentric / ethnorelative dimension of "diversity," one not articulated in the education literature. 
Bennett addresses the ethnocentric / ethnorelative dichotomy in his work. Beginning with a category "denial" in the ethnocentric stages his continuum evolves to a stage labeled "integration" at the far end of the ethnorelative categories. Porter and Samovar's definition of ethnocentrism is cited in Bennett's work. And according to Porter and Samovar (1994), "Ethnocentrism, centeredness on one's own group,...becomes the perceptual window through which a culture interprets and judges all other cultures." (p. 13). By contrast. Bennett defines ethnorelativism as the antonym of ethnocentrism and includes concepts such as Alder's (1977) multicultural person, "a person who is always in the process of becoming a part of and apart from a given cultural context." (p. 26). Unlike ethnocentric individuals ethnorelative individuals engages in evaluation from a number of different relevant cultural perspectives, rather than from only their own cultural perspective. As one progresses along Bennett's continuum from "denial" to "integration" one is thought to develop intercultural sensitivity, or develop from ethnocentric to ethnorelative attitudes. Bennett provides detailed descriptions of each stage including operational definitions and strategies for advancement.

Denial, the first stage at the ethnocentric end of the continuum occurs when "...physical or social isolation precludes any contact at all with significant cultural differences. Since difference has not been encountered, meaning 
(categories) has not been created for such a phenomena." (Bennett, 1986, p.

182). At this point on the continuum, the individual is naive to differences between cultures; he or she remains unaware of the incredible variety of human beliefs, behavior, customs and culture. He or she interprets all events according to only one cultural perspective. This particular category is operationalized in terms of behavior:

...likely either not to perceive difference at all, or to employ wide categories in perceiving difference. An example of the former is the statement sometimes heard that Tokyo (or some other foreign location) is not at all different from, say, New York. ( $p$. 187)

Defense, the second, and middle stage of the ethnocentric end of the continuum, is characterized by Bennett (1986):

...attempts to counter perceived threat to the centrality of one's world view. Because difference must be recognized (and thus given meaning) before it is seen as threatening, this stage represents a development in intercultural sensitivity beyond denial. (Bennett, 1986, p. 183)

Clearly, an individual at this point is caught trying to fend off a perceived threat represented by people different from him or herself. Behaviors characteristic of this stage include, “...statements of hostility....masked by requests for confirmation that one particular group is "really troublesome." (p. 188). Bennett states, "In general, strong appeals to pride in one's own culture probably derive from this stage." (p. 188). At this point one can identify a strong correlation with assimilationist beliefs. An individual in the defense 
stage, not unlike the assimilationist, recognizes differences but interprets them negatively in nearly all circumstances (Janzen, 1994).

Minimization represents the last stage in the ethnocentric end of Bennett's continuum. "Minimization. The last-ditch attempt to preserve the centrality of one's own world view involves an attempt to "bury difference under the weight of cultural similarities." (p. 183). Signs of this stage include statements such as "In other cultures you just have to be yourself." and "There are some things that are true everywhere." (p. 190). At this point along the continuum, cultural differences are trivialized; an individual is aware of variation but elects to interpret difference as unimportant.

Acceptance is the first stage in the ethnorelative half of the continuum. At this point "cultural difference is both acknowledged and respected." (Bennett, 1986, p. 184):

There are two major levels of acceptance that seem to occur in sequence. First is the acceptance of behavioral difference, including language, communication style, and nonverbal patterns. Second is acceptance of the underlying cultural value differences. (p. 184)

At this point individuals are characterized by enjoyment of the exploration of differences. While individuals might ask naive or inappropriate questions, the questions are "geared toward learning rather than confirming stereotypes." (p. 191). Here an individual is engaged in exploration of cultural differences. While he or she may be unfamiliar with differing cultural norms and values, 
unable to empathize, he or she is in the process of gaining information to learn.

Beyond acceptance is adaptation. In this stage an individual is able to “...intentionally shift frame of reference; that is, to empathize." (Bennett, 1986, p. 192). This represents a change of behavior based on the newly accepted difference found in the previous phase. Operational behaviors include generation of appropriate questions regarding cultural differences and the ability to shift one's frame of reference to experience empathy. At this point an individual is addressing issues of difference in cultural values and behaviors and has the capability to consider those differences in action.

The last stage in the ethnorelative end of the continuum is the integration stage. According to Bennett, "The integration of difference is the application of ethnorelativism to one's own identity." (1986, p. 186). Bennett further explains this concept with a quote from Alder (1977) who states the multicultural person is "not simply the person who is sensitive to many different cultures. Rather, he is a person who is always in the process of becoming a part of and apart from a given cultural context" (p. 26). Bennett's operationalization of this stage describes the individual as one characterized by "a lack of strong cultural identification combined with well-developed levels of Acceptance and Adaptation" (p. 193). An individual in this stage is able to 
engage in evaluation based on a number of cultural value systems as well as apply those value systems to his or her own identity and actions.

Bennett's model, while perhaps describing the experience of some individuals, is limited by its assumption that all individuals experience the process of developing from ethnocentric to ethnorelative attitudes in the same manner. Is it not possible that individuals would slip backwards along the continuum due to a particularly negative experience? Or is it not possible some individuals experience this growth in a non-linear fashion? Additionally, Bennett's model accounts for this experience only on an individual level. Is it not possible US society, generally, slips forward and back in response to political leadership or national crises such as the Rodney King beating? Or is it not possible US society develops along this continuum in cultural groups or as regions of the country? This continuum represents only a limited Western linear form of logic.

However, when taken as a taxonomic structure, Bennett's theory provides a promising model. Each of the stages is clearly defined, and for the early stages of the continuum explicit operationalization is provided. However, as one moves to the ethnorelative end of the continuum, operationalization is much more vague. Yet the structure, as a whole, presents a promising taxonomic system. 
In addition to Bennett's continuum which addresses an ethnocentric / ethnorelative dimension of diversity, Lieberman (1994) provides a nearly exhaustive list of lower order concepts included in the currently unarticulated dimension "cultural diversity." Included in Lieberman's list of diversity elements relevant to the classroom are age, gender, occupation, language, religious background, hobbies, socioeconomic class, and dominant cultural background. Not mentioned in this particular list but included in Lieberman's other work are ability and sexual orientation. These categories, together, comprise what appears to be a compilation of the lower order concepts in the "cultural diversity" dimension discussed earlier and can prove useful for creating a descriptive picture of educators' conceptualizations of the dimension.

From the literature search conducted for this study, no operationalization of the learning style differences, pedagogical differences, or language differences dimensions were identified.

Theoretical Assumptions

What remains to be considered are the theoretical assumptions behind the process of educators, or any individual, conceptualizing "diversity." Currently and historically, diversity has been conceptualized both in terms of lower order concepts and constructs. Lower order concepts, are those 
concepts which comprise the larger concept being studied (Chaffee, 1991), while constructivism is an approach whereby meaning is created through the use of bipolar opposites.

According to Chaffee, "Lower-order concepts are closer to the world of observation... A higher-order concept subsumes several lower-order concepts, and the hierarchical organization of this structure of meaning can be imagined as a definitional 'tree." (Chaffee, 1991, p. 25). In other words, the higherorder concept, in this case "diversity," is comprised of several lower-order concepts or elements. These lower-order concepts are more closely associated to observable behavior and should be more easily measured than the broader higher-order concept. In this case, the language style, learning style, and pedagogical differences represent the early lower-order concepts for the higher-order concept "diversity." These elements, (language style, learning style and pedagogical differences) along with others, are assumed to constitute, or make-up, "diversity" for the time. While in any one context one element may present itself as more salient than others, it is the group of concepts which creates the whole, "diversity," within the education literature.

Those who subscribe to the constructivist approach believe, "...the object of scholarly inquiry is the 'world-as-perceived,' not some raw reality untouched by human perception and interpretation" (Smith, 1988, p. 310). For constructivists, "... experience... always requires interpretation; and it is always 
some conceptual scheme, however rudimentary, and not virgin reality, that supplies the criteria by which an interpretation of experience is to be appraised." (Aune, 1967, p. 82.) In other words, the individual experiences reality only through his or her interpretation of that reality. There is no possible way for an individual to separate his or her experience of a situation from some ideal form of that situation. One can only understand reality as one interprets or lives it. Therefore, the epistemological assumption is "...that knowledge is necessarily rooted within individually and socially constituted symbolic structures." (Delia, 1977, p. 69). The implication for the concept "diversity" is that there is no ideal definition of the word, only varying interpretations of the concept, in this case interpretations made by those in the education institution.

Additionally for constructivists, the human is seen as an active agent, shaping his or her own environment, in part through interpretation of events, "The person is conceived as an active agent who reconstructs his environment and who is a source of acts rather than a simple responder to external forces." (Delia, 1977, p. 69). Because of this active human capacity an individual is assumed to engage in the creation of meaning. According to Kelly (1955) an individual "can represent his environment, he can place alternative constructions upon it and, indeed, do something about it if it doesn't suit him." (p. 8). In this statement, Kelly emphasizes both the ability of 
people to actively create meaning of the events that surround them and the human capacity to take action upon those constructed meanings. "Within the framework of constructivism,...interpretive processes are conceived as central to individual and social behavior; all 'variables' are conceptualized within the operation of these central processes" (Delia, 1977, p. 75). Again, the individual actively creates meaning in the context of social interaction, considering individual intentions and with the ability to modify action based on those intentions.

According to Littlejohn, "A construct is a distinction between opposites, such as tall-short...that is used to understand events and things....By classifying an experience into categories, an individual gives meaning to the experience." (1992. pp. 119). Additionally, "Constructs are organized into interpretive schemes, which identify what something is and place the object into a category." (p. 119). In this way, an individual identifies an event, experience or object and classifies it along a number of relevant constructs in order to make meaning of that event, experience or object in relation to similar phenomena. While this categorization of events etc. along relevant constructs is an individual, interpretive process, the creation of the relevant constructs may, in fact, be a social process:

Constructivism...recognizes at base that constructs have social origins; they are learned through interaction with other people. One's construct system is a direct result of a history of interaction in social groups and cannot be divorced from social life. (Littlejohn, 1992, p. 120) 
It is social interaction which determines and creates the relevant constructs an individual uses for evaluation in any given situation. As was indicated previously, "Knowledge is necessarily rooted within individually and socially constituted symbolic structures." (Delia, 1977, p. 69). Therefore, creation of meaning for the concept diversity, or any other concept, is both individual and social, according to the constructivist approach.

"Diversity" as a concept can similarly be understood in terms of bipolar constructs. Historically and currently, the constructs, assimilationism / pluralism and ethnocentrism / ethnorelativism have been utilized to make meaning of "diversity." However, evaluative dimensions such as good and bad, active and passive, growing and dying, and comforting and threatening can be found in the literature and should be considered when developing a measure for the concept "diversity." In addition to these evaluative dimensions, constructs regarding individuals and groups, and personal institutional dimensions are discussed and exist within the literature and require attention. 


\section{CHAPTER II}

\section{METHODS \\ General Methodological Considerations}

The literature review demonstrates that research in the area of teachers' understanding of many concepts, including the concept "diversity," is lacking. When inadequate methods exist to measure a concept and only sparse information regarding the topic is available, qualitative inquiry is particularly useful. According to Patton (1990), "Another reason for using qualitative methods is that for particular outcomes no acceptable, valid, and reliable quantitative measures exist." (p. 130). In fact, because little research has been done regarding teachers' conceptualizations of diversity, little if any quantitative work is available. Crabtree and Miller (1992) demonstrate the utility of qualitative inquiry in situations where information is sparse by referring to two exploratory studies addressing caregivers' experiences, "The fact that both research projects involved the exploration of relatively unknown aspects of caregivers' experiences and decision making meant that qualitative methods were an appropriate choice for gathering data." (p. 206). Additionally, according to Patton (1990), when "...little is known about the 
nature of the phenomenon, qualitative inquiry is a reasonable beginning point for research." ( p. 131).

Qualitative inquiry beings with the phenomenological assumption "...that the perspective of others is meaningful, knowable, and able to be made explicit." (Patton, 1990, p. 278). Unfolding from this perspective is a further "... assumption fundamental to qualitative research--the participants' perspective on the social phenomenon of interest should unfold as the participant views it, not as the researcher views it." (Marshall \& Rossman, 1989, p. 82 ), allowing the informant to set the stage and provide what he or she views as relevant. Therefore, qualitative inquiry is a process of building theory based on grounded data that emerge from observations of respondents' experiences, "Grounded theory depends on methods that take the researcher into and close to the real world so that the results and findings are 'grounded' in the empirical world." (Patton, 1990, p. 67). In such cases theory is built up from real world experiences; experiences come first and theory develops from those experiences.

In order to be prepared for what might emerge and preserve the richness of the respondents' experiences and perspectives it is necessary for the qualitative researcher to enter into research adequately informed and aware of the community under focus yet prepared to adapt to emergent data:

Qualitative inquiry designs cannot be completely specified in advance of fieldwork. While the design will specify an initial focus, plans for observations and interviews and primary 
questions to be explored, the naturalistic and inductive nature of the inquiry makes it both impossible and inappropriate to specify operational variables, state testable hypotheses and finalize either instrumentation or sampling schemes. (Patton, 1990, p. 61)

Therefore, during qualitative research, "Categories or dimensions of analysis emerge from open-ended observations as the evaluator comes to understand..." (Patton, 1990, p. 44). The nature of qualitative research is to identify themes as they emerge from the situation and are revealed by respondents rather than to impose an a priori thematic selection. The interview simply provides the structure through which respondents express their own agendas.

According to Marshall and Rossman (1989), "Data analysis is the process of bringing order, structure, and meaning to the mass of collected data.... a search for general statements about relationships among categories of data; it builds grounded theory." (p. 112). Therefore, the researcher attempts to identify categories of meaning and relationships from the experiences and narrative provided by respondents. Marshall and Rossman (1989) proceed to describe the analytic procedures for qualitative data: organizing information, generating categories and themes, testing emergent hypotheses, searching for alternative explanations for the data, and writing the report. The first mode, organizing information, involves preparing the data for analysis and a general "clean up." This may include transferring the data to 
notecards, coding data according to major content categories or performance of minor editing. The second mode of analysis is described as, "Identifying salient themes, recurring ideas or language, and patterns of belief that link people and settings together..." (p. 116). This process involves reading and rereading of the transcripts, cutting and pasting units of data into similar categories:

The interpretive part involves determining criteria for organizing the textual data into analytically useful subdivisions (in essence coding the data) and subsequent search for patterns within and between these subdivisions to draw substantively meaningful conclusions. A major aspect of the mechanics of qualitative data analysis is equivalent in essence to cutting and pasting: cutting the material apart and pasting the pieces into categorical and conceptual collections. (Morgan, 1993, p. 45)

The third mode of analysis, according to Marshall \& Rossman (1989), involves evaluating the plausibility of the emerging categories or hypotheses against the data. Fourth, the researcher searches for alternative explanations, or engages in "...the critical act of challenging the very pattern that seems so apparent." (p. 119). Testing and retesting the apparent relationships is vital to preserving the integrity of the respondents' experience. Searching for alternative structures ensures an open approach whereby the respondents set the stage and tell the story along with the researcher. Finally, the act of writing the report of findings is performed.

A qualitative research approach implies strengths and weaknesses for the outcomes of research. In-depth interviews are the chief method of inquiry 
for qualitative researchers. Qualitative interviews, whether individual or group, have particular strengths. Through the qualitative interview, researchers can obtain extensive amounts of detailed, specific data in a relatively short time. The interview also allows for immediate follow up and clarification, a luxury not possible with many other approaches (Marshall \& Rossman, 1989).

Additionally, qualitative designs and measures ensure flexibility and creativity (Patton, 1990). However, "...qualitative analysts do not have statistical tests to tell them when an observation or pattern is significant.... This sometimes leads to the making of the qualitative analyst's equivalent of Type I and Type II errors from statistics." (Patton, 1990, p. 406). In other words, because the qualitative analyst must rely on personal judgment (as the instrument of research), he or she may overlook a dimension that is significant, such as a relevant theme. Or, the researcher may attribute significance to something that is not, such as an individual agenda rather than identifying an issue of concern for the respondents.

Issues of validity and reliability are a concern for the qualitative researcher. In the area of qualitative research, reliability and validity are some of the most hotly debated topics.

When conducting qualitative research a researcher is constantly in the research environment testing and retesting the hypotheses in question. Participants are at the heart of the process, constantly being questioned and 
observed and it is the individual or group experience that is valued and sought after. Therefore, the process of constant observation, participation and interviewing while in the field is quite reliable and valid for yielding those results. The qualitative approach focuses on the individual situation, rich description of one situation, and does not attempt to generalize to further settings. The desire is not to generalize to a larger population but to focus deeply on a smaller, unique, setting. This focus redefines the concepts reliability and validity for research. Rather than attempting to reproduce a research situation, as may be the focus of a quantitative study, the qualitative approach attempts to provide a rich description of an experience that can not be reproduced.

As a qualitative researcher, the focus when addressing issues of validity and reliability entails careful thought and documentation regarding decisions and assumptions made (Kirk \& Miller, 1986). It is through documentation and rich description of setting and participants and researcher choices that reliability is established:

First and foremost, fieldnotes are descriptive. They should be dated and should record such basic information as where the observation took place, who was present, what the physical setting was like, what social interactions took place. Field notes contain the descriptive information that will permit the observer to return to that observation later during analysis and eventually permit the reader of the study findings to experience the activity observed through the research report. (Patton, 1990. p. 239) 
It is nearly impossible to replicate a field experience or setting. Yet through the description provided, the reader of research may decide the impacts of decisions made by the researcher.

\section{Methodological Partnership}

The following research project is to be run concurrently with the Graduate Teacher Education Program (GTEP) Children and Communities cohort program evaluation project in the School of Education at Portland State University. There are four distinct and separate research projects occurring simultaneously. However, certain elements of the projects run paraliel (see APPENDIX A).

Each project began with a "Children and Communities Cohort Program Evaluation" survey. Within the survey, exist three distinct content areas. For program evaluation purposes, data was gathered regarding prior knowledge and program influences for the content areas "community," "mid-level students," and "diversity." The survey was created and administered by the cohort research team, comprised of cohort advisors and the research assistant/thesis candidate. At this point, the projects diverge. Each of the four research projects is represented by a separate human subjects application and research program. The "community" research is sponsored and conducted by the cohort advisors. Included in the research plan is a 
focus group and in-depth interviews conducted by the cohort research team, including the research assistant/thesis candidate. The "mid-level students" research will follow the same format, a focus group and in-depth interviews conducted by the cohort research team. The "diversity" research plan is slightly different. Both the cohort advisors and the research assistant/thesis candidate are interested in obtaining and analyzing data regarding the "diversity" content area. As a result, the research assistant/thesis candidate, as well as the cohort advisors, engaged in evaluation of the program data to formulate questions for a focus group and in-depth interviews. Both the cohort advisors and the research assistant/thesis candidate, submitted a human subjects application for the research. The focus group and in-depth interviews were conducted by the research assistant/theses candidate and are the sole property thereof. Initial analysis of the data was conducted by the research assistant/thesis candidate. However, a secondary analysis of the data is to be performed by the cohort advisors with the permission of the research assistant/thesis candidate.

\section{Respondents}

All respondents are a part of the Children and Communities cohort of the state certified Graduate Teacher Education Program (GTEP) in the School of Education at Portland State University. The cohort is a group of students 
who progress through the one year (four quarter) program together attending the same classes and experiencing various teaching assignments concurrently. The Children and Communities cohort began in September 1994 and according to cohort documents (human subjects application 1995) the cohort is designed to provide an experience of high quality practice in elementary schools, develop effective teachers in urban, rural, and suburban environments, develop educational leaders for "multiple diverse communities," and develop teachers capable of providing educative environments that maximize each child's potential and "respect and affirm each child's family, ethnic group and other constructs of social identity."

Students in the GTEP program engage in various coursework and activities over the course of preparation for teaching. Included in this coursework is a class designed to address the issue of "culture contact" and the issues of culture and diversity in the classroom. As a part of this course students were asked to complete a culture contact biography, describing the contact they had experienced with cultures other than their own. Additionally, students were requested to complete a culture contact survey, designed to address these same types of issues.

Following is an excerpt from a human subjects application submitted by the Children and Community Cohort research team. The passage describes in further detail the activities of the cohort over the four quarter program: 
In order to better understand the progress of the cohort, the following sections provide a description of cohort activities by quarter.

\section{Fall 94 Winter 95}

The four issues of this period were: design of the program, selection of sites, admission of candidates and the deployment of resources. Each student was assigned an advisor from the faculty cohort leaders. Prior to the start of the program, an orientation to the program was held. A series of discussions were held with faculty who were affiliated with the cohort.

Week One

Set aside as the first week of the program for cohort orientation, this interval was important to set the tone of the program, establish work in the community as the key to becoming a teacher, establish the context for mid level education, develop contacts with key community entities that influence in and participate in education, and establish a base line of information about the Sunnyside school and its community. One student elected to leave the program. Spring 1995

The goals of this interval were to develop knowledge and skills areas of educative practice for new teachers through courses, projects and field observations. Students were rotated through at least two sites in order to observe the interaction between different schools and their communities and given an opportunity to observe differing age levels of students. At each school, the building principal held an orientation for the teacher candidates. A key objective was to build advisement relationships between faculty and teacher candidates. In planning for the summer experience, a series of meetings was held with summer teaching faculty.

\section{Summer 1995}

Students were involved in university coursework which was not taught by faculty leaders. A series of informal advisory meetings and program feedback sessions were held by faculty cohort leaders. One student elected to leave the program.

\section{Fall 1995}

Students began half time student teaching while continuing to attend classes at the university. Students are asked to videotape a classroom lesson for critique and analysis. Students develop and present a work sample which consists of 10 lessons that they have taught. 
The cohort consists of twenty female and four male students. A total of twenty three individuals agreed to participate in research activities. The average age for students is twenty-six years of age with a range of 22 to $42+$.

\section{Methods Used}

The preceding qualitative approach was used in four major phases for the following research. Beginning with a review of the relevant literature, and an initial survey probe, qualitative questions were developed for a focus group and in depth interviews. This methodology was flexible, however, and questions were adapted according to results of all phases of research. The purpose of this type of research is to generate new hypotheses and information regarding the concept under question from the direct participants themselves.

The decision to utilize a survey, focus groups, and in-depth interviews was made in an attempt to gather data through a number of different channels, thereby minimizing the impact of any one measurement technique. This triangulation process aids in the establishment of validity and lessons the realized and unforeseen consequences of measurement impacts by addressing the research question from a number of angles. While the survey is a written test, requiring accomplished writing skills, the focus group provides an oral method of gathering information from the group as a whole, including 
group opinion. Additionally, the in-depth interviews provide an avenue to gather information from individuals in a way that minimizes the social desirability characteristics found in the focused group interview and allows for the researcher to probe and clarify issues while gathering data in such a way as to privilege lived experience.

\section{Literature Review}

The first phase of the research involved identifying emergent themes from the literature review (refer to APPENDIX B) which were used to develop relevant questions for the focus group interviews, as well as, the in-depth interview protocol. Appendix B summarizes the findings outlined in the Theory chapter.

\section{Survey}

The second phase included the analysis of Question number 31 of the Children and Communities Program Evaluation questionnaire: "Give a definition of 'diversity."' Responses were analyzed and evaluated with an eye to emergent themes.

Survey procedures. Using a student roster, the graduate assistant/thesis candidate randomly assigned each student a number. At this point, the graduate assistant/thesis candidate created a master list containing the student names and code numbers. This list remained in the sole 
possession of the graduate assistant/thesis candidate. The surveys were then coded to correspond with these numbers. At the time of the survey administration, students were provided with a survey containing only their number code in the upper right hand corner of each page. In this way, responses remained confidential where the graduate assistant/thesis candidate was involved and anonymous where cohort advisors were concerned.

Surveys were administered by the cohort research assistant/thesis candidate during a regularly scheduled class session. The survey was prefaced with a standard script (see APPENDIX C). The students were allowed sufficient time to respond and all surveys were completed within twenty-five minutes. One student, upon completion of the survey, elected not to participate in the research. This student's survey was then destroyed. All other students' surveys were collected.

During evaluation of the survey, the open ended question answers (questions 31-34) were detached from the rest of the survey and responses were typed by an assistant not related to the cohort to ensure student anonymity. Next, a code sheet was created and responses were recorded. Survey numbers were recorded with each response to ensure proper tabulation and preserve data for correlations. Then a second code sheet with only gross totals was created. 
Program analysis began with an evaluation of the gross data in an effort to identify emergent patterns. The graduate student/thesis candidate identified areas of potential interest and concern based on an initial reading of the survey. These concerns were presented to the primary research team which discussed, modified and supplemented areas of interest (see APPENDIX D). Question 31 of the Children and Communities Cohort Program Evaluation survey is the main focus of the graduate assistant/thesis candidate: "Give a definition of 'diversity'.". This question was analyzed to identify emergent themes, areas of clarification, and areas of consensus for the focus group and in depth interviews.

Perhaps because question 31 was a small part of a larger questionnaire addressing the issues of diversity, mid-level students and community, the responses given were very brief. Respondents did not engage the topic with much depth. Some responses were only one word, while the longer responses were one or two sentences. None of the responses included more than "listing" of relevant or salient topics. As a result, analysis of emergent themes was reduced to a cataloging of the elements listed.

Qualitative analysis is conceived as an "emergent product of a process of gradual induction." (Lofland \& Lofland, 1995, p. 181), which implies an 
abundance of experiences presented by respondents and structured by the researcher. The emergent themes were placed into a spreadsheet format (APPENDIX E). Among the themes identified were the concepts of "difference" in seventeen of twenty-three responses, "culture," identified in thirteen of twenty-three, "race," and "socio-economic-status" each identified in seven responses and "language" and "beliefs" each mentioned in five responses.

\section{Focus Group}

According to Babbie, (1995) "Sometimes exploratory research is pursued through the use of focus groups, guided small group discussions." (p. 84). Specifically, "...focus groups emphasize the goal of finding out as much as possible about participants' experiences and feelings on a given topic." (Morgan, 1993, p. 7). "The facilitator introduces topics, probes for additional answers, and encourages people to 'piggyback' on others' ideas." (Frey, Botan, Friedman \& Kreps, 1992, p. 129). The focus group has strengths and weaknesses. Morgan (1993) states, "Focus group interviews use group interaction to generate data and gather insights into a research topic that would be less available without the interaction found in the group." (p. 144). In this way, the researcher can hope for an abundance of themes as one may spark another. Additionally, with this method the unit of analysis becomes the group itself as opposed to individuals within the group. However, with the 
group as unit of analysis, comes group pressure, "It may become difficult to distinguish the individual's beliefs from those expressed as a result of group pressures." (p. 144). Based on themes taken from the analysis of the literature as well as the Program Evaluation questionnaire, a number of focus areas were developed for the focus group interviews. A specific protocol was then created, designed to probe into and clarify thematic information gleaned from both the literature search and the Program Evaluation.

Tentative focus group instructions (diversity). "This is the first of two focused group discussions. Topics for this discussion will be Communities and Diversity. In each discussion, ideas will be explored through questions brought forth by the facilitator and fielded by group members. The focus group transcripts are to be utilized for cohort research and for my own personal thesis research which intersects with cohort interests and is why I went to the Children and Communities group. I have just collected forms that indicate each of you acknowledge this discussion is being video and audiotaped, is to be used for research purposes only, and if presented, every effort will be made to mask your identities.

Now I am going to bring to the group a number of topics for discussion. I encourage all of you to talk and contribute to the discussion freely, because each of your opinions is important to the group as a whole. If any of the 
questions are unclear, please tell me so that I may offer some clarification. And feel free to pass on any question which may make you feel uncomfortable.

\section{SECTION I: DEMOGRAPHICS AND EXPERIENCE}

1. Could we begin by proceeding around the circle offering our names and some information regarding how long each of us has been a member of the GTEP and where we are now in the process?

\section{SECTION II: GENERAL DEFINITION}

2. Could you give me your definition of 'diversity?'

\section{SECTION III: GENERAL KNOWLEDGE}

3. When you picture a diverse student what do you see? Probe: Could you talk a little about that?

4. What do you know about diversity and teaching? Probe: Is diversity important for teaching?

5. In your opinion, what, for teachers, are the most relevant characteristics of diversity? Probe: What characteristics are less important? 
6. Does the diversity of students impact the classroom? Probe: If so, how? Probe: If not, why not?

7. What do you, as a teacher, think of students different from yourself?

\section{SECTION III: PROGRAM INFLUENCE}

8. Have you learned about diversity through your experience in the GTEP? Probe: If so, what have you learned? Probe: What activities in the GTEP have helped you to learn the most about diversity?

9. Have you learned skills for working with diverse students through the GTEP? Probe: If so, what skills have you learned? Probe: What experiences in the GTEP have been influential regarding your skills for working with diverse students?"

\section{Pilot Study}

In order to better understand the benefits and limitations of the details of the methodological construction, including the instructions and protocol, a pilot focus group was conducted.

The focus group was conducted as an introductory discussion for a graduate level "Pluralism and Urban Education" class. The class was slightly older than the cohort under investigation, and consisted of approximately twenty students, slightly larger than the typical focus group, of various ages 
and cultural backgrounds. The discussion lasted approximately thirty five minutes and, due to the intimidating nature of having recording equipment present on the first class session, was not recorded.

The class began with a general introduction to the "Plurlaism and Urban Education" course and was followed by a break and the focused group discussion. Students in the group appeared to be quite active in the discussion, exploring ideas and not hesitating to voice agreement or difference of opinion. The group easily created a "working definition" for the concept diversity and had time to move to the next theme for discussion.

The second item for discussion on the protocol is the "picture of a diverse classroom." This item brought some hesitation for this particular group of students. Generally, the group was reluctant to put concrete examples to what is considered diversity. Comments in response to the request for a picture included things such as "It is difficult to describe what it might look like."

As a result of the pilot study, some slight modifications were made to the focused group protocol. The beginning section on demographics and experience has been deleted due to the subjects' familiarity with each other as a result of the extended cohort experience. Additionally, the "GENERAL DEFINITION" section question was modified to include a brief introduction: "This cohort training program has used the concept of diversity as a theme. 
Because it is an important part of the group experience, could you talk to me about your definition of diversity?" The next question was also modified to include an introduction: "We have talked a bit about an abstract sort of definition for the concept diversity but l'd like to see if we could develop a more concrete picture. You all have had quite a bit of experience in the classroom now. If I were to ask you to picture a diverse classroom, what would you see?" Finally, questions four and five were collapsed to form the question "What do you believe the relationship between diversity and teaching should be, if there should be any relationship at all?"

Revised focus group instructions (diversity). "This is the first of two focused group discussions. Topics for this discussion will be Communities and Diversity. In each discussion, ideas will be explored through questions brought forth by the facilitator and fielded by group members. The focus group transcripts are to be utilized for cohort research and for my own personal thesis research which intersects with cohort interests and is why I went to the Children and Communities group. I have just collected forms that indicate each of you acknowledge this discussion is being video and audiotaped, is to be used for research purposes only, and if presented, every effort will be made to mask your identities. 
Now I am going to bring to the group a number of topics for discussion. I encourage all of you to talk and contribute to the discussion freely, because each of your opinions is important to the group as a whole. If any of the questions are unclear, please tell me so that I may offer some clarification. And feel free to pass on any question which may make you feel uncomfortable.

\section{SECTION I: GENERAL DEFINITION}

1. This cohort training program has used the concept of diversity as a theme. Because it is an important part of the group experience, could you talk to me about your definition of diversity?

\section{SECTION II: GENERAL KNOWLEDGE}

2. We have talked a bit about an abstract sort of definition for the concept diversity but l'd like to see if we could develop a more concrete picture. You all have had quite a bit of experience in the classroom now. If I were to ask you to picture a diverse classroom, what would you see? Probe: Could you talk a little about that?

3. What do you believe the relationship between diversity and teaching should be, if there should be any relationship at all? Probe: What characteristics of diversity are less important to this relationship? 
4. Does the diversity of students impact the classroom? Probe: If so, how? Probe: If not, why not?

5. What do you, as a teacher, think of students different from yourself?

\section{SECTION III: PROGRAM INFLUENCE}

6. Have you learned about diversity through your experience in the GTEP? Probe: If so, what have you learned? Probe: What activities in the GTEP have helped you to learn the most about diversity?

7. Have you learned skills for working with diverse students through the GTEP? Probe: If so, what skills have you learned? Probe: What experiences in the GTEP have been influential regarding your skills for working with diverse students?"

Focus group procedures. Respondents were part of the Children and Communities cohort and were informed of the focus group through announcement in regularly scheduled classes as well as by written correspondence. Subjects volunteered for participation in both the focus group and the in-depth interview process. As the group was convened students were asked to sit in a circle around a table with recording devices. Students in the focus group were informed of their ability to withdraw from the discussion and were to be provided with a standard informed consent form 
containing mention of the video and audio taping (see APPENDIX F). Additionally, the informed consent form included mention of students' inability to remain anonymous due to the use of the video equipment. However, students were assured anonymity if the tape is to be used for other than evaluation purposes. At this point, the video camera was started. The focus group facilitator was the graduate assistant/thesis candidate and cohort leaders were also present. At this point the facilitator began the protocol/instructions. When the group had discussed for approximately eighty minutes, the facilitator stopped the group, asked for a summative comment from each group member, and thanked all the participants for their contributions. At this point, a written form was distributed requesting input regarding topics that participants felt were not fully explored through group discussion or contributions individuals felt they were unable to make. The camera was stopped and the tape was stored in the office of either the graduate research assistant/thesis candidate or one of the cohort advisors when not in use.

The focused group interview was held in the lounge of the Campus Ministries building on campus at Portland State University. This location was chosen to provide a less formal atmosphere than found in the classroom. Because instructors from the cohort were to be present at the time of the interviews, the researcher felt it imperative that the atmosphere of the 
interview be removed from that of the "graded" classroom (APPENDIX G). Additionally, the discussion time fell during the middle of a "100 year" flood which weighed heavily on the minds of all involved. The researcher felt it important to create a safe, inviting atmosphere where individuals could concentrate on the subject at hand amidst the outside chaos. The room was larger than most classrooms, and had large entrance and exit arches providing a lounge type atmosphere. There were, couches, large chairs, several coffee tables, end table lamps, many windows and a fireplace in the carpeted room. Additionally, cookies, breads, and juice were provided. Students sat in the couches and large chairs around two square tables. The elongated circle was selected to promote an equal power seating arrangement, indicating all contributions were to be equally valued.

The audio recording devices were set in the middle of the tables and tapes were turned over once during the discussion. Additionally, one of the cohort advisors stood outside the elongated circle at one end and videotaped the discussion with minimum movement. This was done to provide least possible distraction while videotaping the discussion for more reliability. In this way, the exact question phrasing, group nonverbal cues, and researcher choices are preserved. 
In the time provided for each focused group discussion, only two of the many issues for consideration in the protocol were covered. The discussions were conducted much like a semistructured interview in that, because the discussion was being recorded to provide a measure of reliability, the researcher allowed the format to unfold as the participants felt comfortable rather than attempting to control the flow of conversation. Similar issues were addressed in each discussion, yet the questions were not posed in an identical manner. This allows for flexibility and the ability to utilize the experiences of the respondents in creating an interview question.

Each group covered only two of the many issues proposed on the original protocol: SECTION I: GENERAL DEFINITION 1. This cohort training program has used the concept of diversity as a theme. Because it is an important part of the group experience, could you talk to me about your definition of diversity? SECTION II: GENERAL KNOWLEDGE 2. We have talked a bit about an abstract sort of definition for the concept diversity but l'd like to see if we could develop a more concrete picture. You all have had quite a bit of experience in the classroom now. If I were to ask you to picture a diverse classroom, what would you see? Probe: Could you talk a little about that? 
My assumptions as researcher. The researcher assumed during the focused group discussion individuals would provide a number of "laundry list" types of answers, largely addressing the cultural diversity dimension of the literature. This assumption is based on the idea that it would be easy for a focus group to "list off" groups that might be included in or demonstrate the concept diversity without engaging such a difficult and multifaceted topic. This also leads to the assumption that the focused group discussion may choose to take an easy route through discussion and finish as quickly as possible. This arises from the fact that the discussion was scheduled for a Saturday, a time which most students do not spend at school.

Based on experience discussing the issue of diversity with students and teachers, the researcher did not expect individuals to discuss diversity along the ethnocentric / ethnorelative continuum. In the researcher's opinion, this appears to be a highly abstract, though interesting, continuum of development not contemplated by "non-academics." However, the researcher did expect it was possible participants would utilize an ethnocentric / ethnorelative continuum as a typography, indicating some individuals behaved in an ethnocentric, and others in an ethnorelative, manner with still others somewhere in the middle. 
Additionally, an overt evaluative dimension was not expected to be discussed due to the nature of group discussion and social desirability effects. As a result of the discussion of such a politically and emotionally charged topic, it was a concern of the researcher that little engagement of the topic would result from a group discussion. The researcher's opinion is that the concept "diversity" is a complicated, multifaceted, often contradictory concept that is a great cognitive load to engage.

It was expected, however, that concrete examples and narratives would be used to demonstrate points and ideas. Because the cohort had just finished student teaching prior to the focused group discussion, it was expected that life experiences related to the topic of discussion would be at the forefront of individuals' minds.

After the focus group the researcher was surprised at the variety of responses provided during group discussion. The original expectation was too restrictive, as each focus group moved along its own horizon. Additionally, the expectation of many concrete examples was met and exceeded. Both focus groups were sufficiently capable of applying the abstract list of elements discussed to their own experiences and providing examples. Beyond the exceeding number of examples provided, the researcher was surprised to find the complexity with which the topic of 
discussion was addressed (see CHAPTER III). Cohort members identified many levels of meaning for the concept and clearly identified the complexity inherent in operationalizing such a multifaceted idea. The assumption of the discussion taking an easy route due to the timing of the meeting was demonstrated to be a false assumption.

Due to time constraints, the decision was made to have the focused group interview tapes professionally transcribed. This impacts the researcher's ability to identify the fine nuances of discussion. However, the researcher viewed the video tape several times prior to beginning evaluation or reading the transcripts. Additionally, the transcripts were checked for accuracy including editorial comments and grammatical errors. Any small errors identified were rectified.

Analytical choices. The researcher read through the transcripts several more times before attempting to identify emergent patterns. It is at this point where the challenge begins for the researcher. According to Patton (1990), "There are no ways of perfectly replicating the researcher's analytical thought processes. There are no straight forward tests for reliability and validity....do the very best with your full intellect to fairly represent the data." (p. 372). With this in mind the researcher turned to Guba's (1978) assertion. According to Guba, the investigator must begin analysis by searching for "recurring 
regularities" in the data. Then the researcher maximizes the extent to which categories hold similar within, "internal homogeneity", and are different across, "external heterogeneity", and moves to a process of vacillation between the meaningfulness of the classification categories and the placement of data within them. With this advice, the researcher began the process of analysis.

The researcher identified many examples for attempting the analytical process, yet none identified provided the structure desired. As a result, the researcher attempted the aforementioned process in a slightly modified manner. The transcripts were read several times and emergent themes or categories of meaning were identified with an eye to a most exhaustive list. At this point a spreadsheet was created with these themes at the head of columns. Then the transcripts were reviewed again with these themes in mind and specific passages of data identified, according to transcript line number, and placed in the spreadsheet accordingly. At this point the many categories were analyzed for "internal homogeneity" and "external heterogeneity" and collapsed to represent more mutually exclusive units.

\section{In-Depth Intervielws}

From this point, the in-depth interview protocol was developed based on information emergent from the focus group, Program Evaluation and literature search. The in-depth interview was intended to focus more deeply 
on specific content areas and themes identified as salient and relevant through the previous phases, "The purpose of interviewing, then, is to allow us to enter into the other person's perspective. Qualitative interviewing begins with the assumption that the perspective of others is meaningful, knowable, and able to be made explicit." (p. 278). Therefore, the "...intensive interview seeks to discover the informant's experience of a particular topic or situation." (Lofland \& Lofland, 1995, p. 18). Again, the in-depth qualitative interview has strengths, including a wealth of data and the ability to probe and clarify immediately and weaknesses such as interviewer bias, and dishonesty on the part of the interviewee.

Tentative in-depth interview protocol (diversity). "I have just reminded the interviewee of his or her rights under human subjects regulations and have verbally received a second confirmation of permission to record this interview. I am going to ask you a few questions related to the questions posed during the focus group discussion. If you feel any of the questions I have asked are unclear, please tell me so that I may clarify. If any question causes you to feel uncomfortable, feel free to pass on the question.

\section{SECTION I: DEMOGRAPHICS AND EXPERIENCE}

1. Before I get started could I get your age for demographic records? 
SECTION II: GENERAL DEFINITION

2. One of the questions that we discussed in the group was a definition for the concept diversity. So to get started I was wondering if you could talk to me a little bit about a definition you might have for 'diversity?'

\section{SECTION III: GENERAL KNOWLEDGE}

3. So after we talked about a definition, we got a little more concrete with the question, 'When you picture a diverse classroom what do you see?' Can you remember what you thought about for this question? Probe: Could you talk a little about that? Probe: What don't you see?

4. What do you think the relationship between diversity and teaching should be, if there should be any relationship at all? Prompt: Looking back to the definition of diversity, are there any aspects which are more important to the relationship between diversity and teaching? Are there any aspects that are less important?

5. How about the classroom? You have spent a while in the classroom now, in your experience, does the diversity of students impact the classroom? Probe: If so, how? Probe: If not, why not?

6. Is it important to learn about diversity as a teacher? Probe: If so, why? Probe: If not, why not? 
7. In your opinion, what, for teachers, are the most relevant characteristics of diversity? Probe: What characteristics are less important?

In-Depth Interview Procedures. From the focus group participants' responses, and the questionnaire distributed subsequent to discussion, three individuals were selected to participate in the in-depth interviews. Students were contacted by phone and requested to participate in the interview. The interviews were conducted in a conference room at Portland State University and were audio taped. Because the informed consent form provided at the time of the focus group interview had two parts, a separate informed consent form was not provided at this time. The researcher reinformed the subjects of their rights to end the interview at any time and, again, verbally requested permission to record the interview. At this time, the interviewer started the protocol. The interviewer was the research assistant/thesis candidate. At the end of the interviews, the interviewer thanked the subjects for their participation and the tapes were stopped. Again, the tapes were stored in the office of the graduate assistant/thesis candidate or the cohort advisors when not in use.

In an effort to identify individuals for the in-depth interviews, the researcher received a list of cohort members available at the time of the interviews (as the cohort process ends at the start of the spring quarter, many 
individuals had left the area for other activities by the time the in-depth interview process began in late March.) All of these individuals were contacted by phone and requested to donate an hour of their time to the interview process. Individuals were notified the interviews would cover similar material as past research and would echo the focused group discussion. Additionally, individuals were provided the opportunity to select the time and location of their interview. In this way, it was hoped individuals would be most likely to volunteer for the interview process and would be most comfortable and free to speak at the time of the interview. All locations were to be private enough for candid discussion and quiet enough to ensure the ability to record conversation.

The interviews took place on campus at Portland State University in a small room, much like would be used for an instructor's office. The room was painted an institutional white, although covered with dirt from a lack of maintenance, had grayish tiles, contained two desks like would be found in a classroom (chair attached), and had no windows. However, it was small enough to be intimate and private, and the interviewee was aware of the location prior to the interview. Both the interviewer and the interviewee sipped on juice or soda throughout the interview and the atmosphere appeared to be easy. 
My assumptions as researcher. Prior to conducting the interview process, the researcher expected difficulty recruiting respondents for the interviews. Because the cohort training program had ended before the start of the interview process, the researcher expected many individuals to be out of the area or no longer interested. However, to the surprise of the interviewer, two individuals responded after the first call and a third responded after the second call. There were no other responses to the second call.

In addition to the difficulty identifying respondents, the researcher expected the interviews would be somewhat more formal than desired. Because the researcher had been visibly associated with cohort advisors throughout the cohort process, and had, in fact, conducted a lecture during class time, the expectation prior to interviewing was that the relationship between interviewer and informant would be somewhat of unequal status. It was expected that this dynamic would impact the flow of conversation and the willingness of respondents to disclose. To combat this issue, the interviewer wore casual clothes (jeans and a sweatshirt), discussed the audiotape prior to entering the interview room, offered and served respondents juice or soda, and sat in a relaxed or slouched position within the chair.

The researcher, however, did expect a number of concrete examples to be provided, as in the focused group discussion, especially with regard to the 
questions about mixed messages and standards in the classroom. The researcher expected the orienting questions to allow the informant time to orient to the discussion, leading to a good flow of conversation by the time issues of mixed messages and standards were raised.

The expectation regarding the formality of interviews was, in some cases correct and in others unfounded. During the first interview, the informant remained fidgety in her chair and guarded throughout the discussion, the main concern and expectation of the researcher. However, both the second and third respondents began the interviews in fairly rigid, formal, posture and began to relax as the conversation progressed. In each of these cases, the interviewers and respondents carried on informal conversation regarding other topics after the interview tape had stopped. This was not the researcher's expectation, however, it was a pleasant surprise. The second and third interviews flowed smoothly with respondents sharing rather freely.

Again, as with the focused group discussions, the researcher expected respondents would engage the topic of "diversity" only superficially, due to the complexity of the topic and the desire to cut the interview short. The researcher believes discussion of such a politically and emotionally charged topic to be a large cognitive load which many individuals would rather avoid. 
The researcher assumed it would be difficult to engage respondents and require several probes to begin the conversation. In some interview situations this was the case. Particularly during the first interview, with Alison, the researcher felt as though the conversation remained quite superficial throughout. However, during the second and third interviews, the conversation engaged the topic with much more depth. Generally, the researcher believes this occurred as a result of improving researcher interview skills as much as the respondents' desire to address the question.

Respondents. All total there were three respondents, Alison, Barbara, and Chad (pseudonyms). Each interview was conducted in the same room and each interview lasted approximately forty-five minutes.

Alison is a white female approximately twenty-six years old with dark, straight hair, cut slightly above the shoulders. She has a pasty white face with freckles and dark sparkly eyes. During the interview she wore dark clothes with a dark, waist-length jacket and a T-shirt. She sat in the desk with her juice, leaned over the table and remained fidgety throughout the interview. Her legs were crossed and she bobbed her top leg.

Barbara is also a white female approximately twenty-three years old. She has shoulder length brown hair with a half-grown permanent, large eyes and straight white teeth. Her skin is pale and clear. During the interview she 
wore a denim jacket and a T-shirt without a logo, jeans a similar color to the jacket and tennis shoes with short white socks which showed as she crossed her legs. She drank a soda throughout the interview as she sank into the back of her chair.

Chad is a black male, twenty-six years old. He has short, dark hair and dark, tired eyes. His skin is dark and clear. He has sparkling white teeth with small gaps between in the front. During the interview, he wore a black leather jacket, black slacks, black socks and dress shoes. With this he wore a knit sweater with a light colored dress shirt beneath. He choose not to drink a beverage during the interview. He started the interview rather fidgety and avoided eye contact. However, by the end he was leaning back in his chair and maintaining eye contact.

The interview protocol. The protocol for the interview was modified, as is often the case in qualitative research. Researchers commonly modify and redefine the focus of research as respondents identify relevant issues. A beginning orientation question was added to provide an opportunity for the interviewee to reflect on the focused group discussion process: "So what did you think of the focus group discussion you were in?"

Because the focused group discussion and the survey format were both limited to a small number of research questions or issues, these issues 
were, again, included in the in-depth interviews to probe, clarify and receive an additional measure. Additionally, because a definition of diversity and diversity in the classroom are issues with which the cohort is familiar, the repeated questions were placed at the beginning of the interview to provide additional orientation questions and allow the interviewee to begin to focus on the issue under study. These questions are questions one and two from the focused group discussion: SECTION I: GENERAL DEFINITION 1. This cohort training program has used the concept of diversity as a theme. Because it is an important part of the group experience, could you talk to me about your definition of diversity? This was modified to read "One of the things we talked about in the focus group was a definition for diversity. So to get started I was wondering if you could talk to me a little bit about what you see as the essential elements of this concept diversity after your teaching experience?" SECTION II: GENERAL KNOWLEDGE 2. We have talked a bit about an abstract sort of definition for the concept diversity but l'd like to see if we could develop a more concrete picture. You all have had quite a bit of experience in the classroom now. If I were to ask you to picture a diverse classroom, what would you see? Probe: Could you talk a little about that? Probe: What don't you see?

Beyond these questions, it was decided the in-depth interview would continue to probe themes emergent from the focused group discussion, 
including: mixed messages, issues of standards, community involvement, how one might teach diversity, and the issue of race and its assumption as a primary issue.

Because the issues of standards and expectations were raised in the focused group discussion several times, yet few examples were provided, the interviewer decided to include a line of questioning to follow up on these issues. Specifically, the interviewer chose to ask: "I thought it was interesting in the focus group when the discussion moved to the idea of diversity and standards and special expectations. What did you think of that? How do you think these relate to the topic diversity and the classroom? Probe: Do you think cooperation and competition fit in here?"

In addition to standards and expectations, the group discussion opened the issue of diversity as an ongoing experience. This issue was also raised in the interview guide. Specifically, the question "One of the other things that was talked about in the focus group was diversity as something that should be 'ongoing'. Do you have any ideas about this from your experience?" was asked.

The concept of diversity as a complicated issue that often involves mixed signals was raised in the group discussion as well. This issue was raised in the interview as "You know, one of the things I heard the group 
talking about quite a bit, as if you all seemed to understand each other, was the notion of diversity being related, in your experience, to mixed messages. I am a bit unclear about what that might mean to you. Do you have any ideas?"

Community and diversity also appeared to be linked in some manner that was never fully fleshed out. Because the focused group discussions for community and diversity were conducted back to back, community may have been assumed to be connected with the concept diversity. However, what connections there may be were not clearly stated. As a result, this issue was raised explicitly during the in-depth interviews. The question "In your experience, do you think community and diversity are connected to one another?" was asked.

Diversity was also assumed to be a concept that can be taught in the schools. The examples provided for methods of teaching this concept were experiential in nature. Yet not many examples were provided and, thus, this is an area for further inquiry. The question was posed as follows: "Oftentimes during our discussion, the issue of teaching diversity was raised. As a teacher, how might one go about teaching diversity?"

The last, and perhaps the most problematic, issue raised during the discussion was the issue of race. During the group discussion, it was stated that race is assumed to be one of the most commonly considered components 
of diversity. Yet, during the discussion, the issue of race was raised only on this one occasion. It is unclear how the issue of race relates to the concept diversity within this group. As a result the issue of race will be raised during the in-depth interviews for clarification.

The final area of questioning was a reflective question about the process. The respondents will be asked their opinions about the experience of discussing these issues both in a group format and in a private, one-on-one interview, "What did you think of the experience of discussing these issues in a group? How did that compare to this discussion we have had?"

Revised in-depth interview protocol. I have just reminded the interviewee of his or her rights under human subjects regulations and have verbally received a second confirmation of permission to record this interview. I am going to ask you a few questions related to the questions posed during the focus group discussion. If you feel any of the questions I have asked are unclear, please tell me so that I may clarify. If any question causes you to feel uncomfortable, feel free to pass on the question.

1. "So what did you think of the focus group discussion you were in?"

2. "One of the things we talked about in the focus group was a definition for diversity. So to get started I was wondering if you could talk to me a little bit 
about what you see as the essential elements of this concept diversity after your teaching experience?"

3. "We have talked a bit about an abstract sort of definition for the concept diversity but l'd like to see if we could develop a more concrete picture. You all have had quite a bit of experience in the classroom now. If I were to ask you to picture a diverse classroom, what would you see? Probe: Could you talk a little about that? Probe: What don't you see?"

4. "I thought it was interesting in the focus group when the discussion moved to the idea of diversity and standards and special expectations in the classroom. What did you think of that? How do you think these relate to the topic diversity and the classroom? Probe: How do you think cooperation and competition fit in here, if at all?"

5. "One of the other things that was talked about in the focus group was diversity as something that should be 'ongoing'. This was something that was unclear to me. Do you have any ideas about this from your experience?"

6. "You know, one of the things I heard the group talking about quite a bit, as if you all seemed to understand each other, was the notion of diversity being related, in your experience, to mixed messages. I am a bit unclear about what that might mean to you. Do you have any ideas?" 
7. "In your experience, do you think community and diversity are connected to one another?" was asked.

8. "Oftentimes during our discussion, the issue of teaching diversity was raised. As a teacher, how might one go about teaching diversity?"

9. "Race was also talked about a little bit during our group discussion. How do you see race fitting in a diverse classroom?"

10. "What did you think of the experience of discussing these issues in a group? How did that compare to this discussion we have had?"

During the first interview, the interviewer added an additional question which targeted the general importance of and definition for the concept under question. The question "If you were to give a new teacher in training some advice regarding diversity in the classroom, what would you say?" This question was added to the protocol for subsequent interviews as well.

The preceding protocol was used to conduct three in-depth interviews which were then analyzed by the researcher in an effort to identify salient themes. The results are presented in CHAPTER III. 
CHAPTER III

\section{RESULTS}

In an effort to better understand pre-service teachers' conceptualizations of "diversity," two focused group discussions and three indepth interviews were conducted. The discussions and interviews were transcribed and analyzed, using qualitative methods (see methods chapter) to identify themes of discourse. The themes identified through analysis on the part of the researcher are presented below. It should be noted the results of this inquiry mirror, in many ways, the debate regarding diversity, generally. The discussions conducted were complicated, multifaceted, and at times contradictory.

\section{Focus Groups}

After reading the transcripts of the focused group discussion (APPENDIX $\mathrm{H}$ ) several times and distilling the conversation in an effort to better understand and represent the data, the researcher formalized a spread sheet including themes emergent during conversation (see APPENDIX I). The spreadsheet contains a number of thematic headings under which are 
line numbers which correlate with passages in the transcripts. It is this data which is described below.

\section{$\underline{\text { Structures }}$}

One of the major areas of discussion during both focus groups was diversity as it relates to societal structures. While this particular phrase was not identified during the conversations, numerous specific structures were discussed including special expectations in the classroom, school and state standards, teaching styles and learning styles, and cooperative versus competitive structures in school and mainstream US society as a whole. As a result, the researcher chose to identify the category as diversity relating to structures.

Specifically, diversity was operationalized as a need for flexible structures and expectations within the classroom. Cohort members expressed a need for adaptation and modification of classroom standards:

(speaker 1)I set parameters up, to include the siblings at the top, your parents and your, and you know, grandparents - I had to set parameters, or the kids would, you know - but this one child, to him and in the Asian Community, cousins, and aunts \& uncles are also included in there so his whole tree was covered. And my mentor teacher didn't - I mean she's like "You can't even see his tree." And I thought, well, you know, this is his family tree to him and I have to let him do that, I have to accept that, you know, he needs to go beyond my parameters because, you know - there's two, and, I don't know, I just thought, you're teaching it, but you're not really accepting it yourself too. Even though you set parameters, you have to be willing to be - (speaker 2) Flexible. Flexible. Flexibility, key word. (laughter). 
Generally, respondents felt it necessary to provide flexibility of standards in the classroom in order to have "diversity."

Flexibility of expectations was also extended to grading of students.

Some cohort members expressed a necessity to provide a flexible curriculum standard:

(speaker 3) ...we have some modified programs for some of our kids that don't learn as fast as the other ones, so we cut down some of the - and they can do great on their work, on their modified work that they turn in, they can get an A on the work they turn in...And um, some kids' levels just aren't as high as others'. To help us help them, we cut their work in half so they can spend a little more time with their classwork. And, I mean, for the most part it works well.

Another individual said, (speaker 1) "It's really hard to specialize your expectations for each student. But you feel like you have to...it feels like it's the right thing to do...".

The issue of diversity as a set of flexible standards extends beyond the classroom as stated by cohort members. In addition to their concern regarding classroom flexibility, cohort members expressed a concern for flexibility with school and state education standards as well:

(speaker 1) And the standards, too...you're compared to everyone else, there's no focus on, "You can do this and this is what you do, and that's ok". There's no....you're compared with, "in seventh grade, you're supposed to know this." They come out with those books now that say, "What your first grader is supposed to know." And really, what you know in first grade is ok, and what you accomplish in first grade is great for you, and that's fine. But we have this standard of...(speaker 2)You need to know this by first grade if you're going to college - if you're going to MIT - (laughter). 
The sentiment of frustration with rigid state standards and the ways in which

these standards do not provide for diversity in the classroom was clearly

evident. Similarly, cohort members felt the use of standards impacted the

learning environment of the classroom:

(speaker 4) We're doing the same thing; our math scores at our school were low, and so they're basically telling us they want us to teach the kids to the test. They want us to teach them the stuff that's gonna be on the test so they can bump those scores up. And that just goes against everything...(speaker 5)Ya, the heck with all the diversity and all the levels, you have one level - to get the school on a high level for that test. That's all that counts.

Cohort members identified varying teaching styles and learning styles as a part of diversity:

(speaker 2) kids have advantages brought into school in a situation like that can be positive if the kids, um, that are getting the modified work can make the connection between, you know, "I did this work and I'm proud of it...." (speaker 6)... if you look at the way schools are structured, what we value is a certain standard of education that isn't - , that's very book-oriented education, it isn't a value on other types of you, know, being a whole, complete person, it's about a certain educative standard and that's what we value.

In this situation, respondents appeared to correlate different learning styles and teaching styles with the presence of diversity.

Competition and cooperation as structures in the school system and in mainstream US society intersected with the concept diversity for cohort members as well. Cohort members indicated diversity correlates with a cooperative structure in the classroom and in the larger societal realm. When 
asked if any one part of the concept diversity stood out as particularly important, one individual stated, (speaker 1) "Everybody has their own thing, um, to offer to larger groups, everybody has their own strengths, they um, to complement each other. You know.". Standards were also viewed as competitive and in opposition to flexibility and diversity in the classroom, (speaker 1) "And the standards, too...you're compared to everyone else, there's no focus on, 'You can do this and this is what you do, and that's ok'. There's no....you're compared with, 'in seventh grade, you're supposed to know this'." Still another individual indicated (speaker 2) "...diversity is a good concept, we should teach it...I think it's good to teach kids to work together, that's the most important thing to me. It's not what they see, it's what they see is that they've got to be better than the others." The theme of cooperation and competition was, perhaps, most clearly stated when speaking of the school's attitude about the competition associated with standardized tests, (speaker 5) "Ya, the heck with all the diversity and all the levels, you have one level-to get the school on a high level for that test. That's all that counts."

When cooperation and competition were generalized to mainstream US society, individuals indicated their concern:

(speaker 6) As far as the big picture is, I really respect what both of you are saying - unfortunately, I really don't see society as a whole or America as a country really changing their opinion about - You just turn to one factor - that is the population's just going to explode more than it is now. There's so many more people looking for jobs, trying to scratch out a living, feed themselves, $I$ 
don't see that society is going to let up and say, ok, let's accept people's diversity, let's, let's cooperate with one another.

The individual went on to state "I don't see society changing its opinion about competitive standards."

\section{Types Of Diversity Or Criteria}

Many times during the focused group discussion individuals devoted time to enumerating who was included in the concept diversity. Diversity was addressed as if there were different types of diversity or as though an individual or group might fulfill a certain criteria which qualified under the concept. As a result, the researcher identified these statements as a separate emergent theme "types of diversity or criteria."

Perhaps the most salient of the criteria to emerge from the discussion was the criteria of difference. One cohort member went so far as to state that diversity was not "real" diversity without differences, (speaker 1) "...but diversity there is not diversity. It's not differences - essentially, how I think of diversity, is there are differences." In addition to this comment, there were many others:

(speaker 7) "And some of them had brought out samples of foods from, um they weren't to eat, just for show, different countries; others had picture books. I mean, you know, there were kids from Vietnam, obviously, um. Armenia, um, a, Russia, Ethiopia, um - I mean it just - It was endless - I had no idea that we had so many people from so many different places at (school indicated).

Others echoed this comment:

(speaker 8) ...celebrated the diversity of people where it first started off with everyone has different eyes, and it showed 
everyone with different noses. It just went through everything. At the end, it talked about how, um, you know, everyone has different colored skin and how we're all beautiful and how different people have different kinds-and it went... (speaker 8 ) When I think of diversity I think of looking at different cultures... (speaker 5) all the diversity and all the different levels....

In addition to the criteria of general difference, academic differences

were raised as one type of diversity. One focus group member spoke of the experiences he had:

(speaker 3) The diversity I see most is the academic - it's the range and level at which kids learn, there's more than one level in the sixth grade class it's like there's such a huge span of, of what grade you're in. There's kids always at the bottom, some kids at the top and just a bunch in the middle.

Examples of academic difference were most prevalent when discussing academics as a part of diversity. Cohort members discussed students at different achievement levels, modified programs for students, grading students of different academic abilities, and student performance in the classroom:

(speaker 1)I guess I can see that because when like you've got all these papers, kids, you know, with different levels of achievement and different accomplishments and different knowledge. Tracy can't read, and so-and-so can read... (speaker 3) Ya, we cut down their workload, cut in half from what the other kids get, so even if they do their work and do it good, they get an A. But the other kids always remind those kids, 'cause those kids always seem to figure out which kids are on the program - 'cause they always find out what kids are being helped and that kind of stuff, and 'cause kids will come up to me afterwards, l'll say, good job, you're doing really good, and they'll say, Yeah, but it's not the same as, you know, Joe over here, you know, who's doing all the work. (speaker 1) And um, some kids' levels just aren't as high as others'. 
Generally, cohort members identified academic differences as a primary component of diversity.

Race, as an issue was raised by cohort members as a primary component as well. While there was not much discussion of the issue, when it was raised cohort members agreed it was a vital part of the concept which, in fact, often overshadowed other parts, (speaker 8) "I think I know... when you think of diversity, a lot of it is race, you the uh, but also it's more than that, it's background, it's learning abilities, it's talents, it's so much more.". Cohort members felt race, while important, was far from the sole, relevant component of diversity, (speaker 7) "Well, actually, it was very similar to what (name) was saying, was, I think we tend to only think of diversity as race - I'll leave it at that But that isn't the case. She explained it so well. (laughter)".

In addition to these criteria which were discussed at length, many other criteria were raised during the course of the focused group discussion but were not addressed in detail. Among these criteria were the concepts of, ethnicity, culture, gender, economics, ability, background, talents and language.

\section{Mixed Messages}

One of the clearest themes to emerge from the focused group discussion was the topic of diversity and mixed messages. Cohort members felt as though diversity is highly correlated with mixed messages and inconsistent practices in 
their experience. This idea was clearly expressed by one individual in the following passage:

(speaker 1) And I thought, well, you know, this is his family tree to him and I have to let him do that, I have to accept that, you know, he needs to go beyond my parameters because, you know there's two, and, I don't know, I just thought, you're teaching it, but you're not really accepting it yourself too.

Others echoed this sentiment loudly by indicating similar experiences:

(speaker 2)If you talk about diversity being important, um and we talk about like - but teachers are put into a problem... diversity, teachers are feeling that... reality kids, kids see that that's... understand that there's mixed signals as far as diversity is concerned, you know, saying diversity is good, but yet, that's not really what's happening, diversity is not, you know, the message is being sent that it's not. It's better to be one way, it's better to be the strongest, you know, that's it. So you can say diversity is good, but it's not happening. It's not being practiced. So you're giving mixed signals - the teachers are in difficult positions as far as that's concerned... diversity's good. I think everybody agrees. We all agree that it's good, but - I mean you know, you come to the point where you've got to make a decision about how much time you spend with the kids that have learning problems; the resources the budget cuts and always a certain amount of students are gonna get .... diversity is a good concept, we should teach it, but it can be counterproductive in some respects if it's not, if it's not the whole of the parts, the whole thing so I think it's good to teach kids to work together, that's the most important thing to me. It's not what they see, it's what they see is that they've got to be better than the others - um - It's a tough thing. Diversity, it's a tough thing.

(speaker 1) In a sense, so what you're saying, is the real world doesn't practice what we preach? At all? We can teach them about diversity, and talk about diversity and use diversity in the classroom but when we get outside, it's different. (speaker 6)Um, we can try to teach kids different ways, and vary our methods of how we approach these kids, but what it comes back is in this society and in this world, and the way it is ingrained, what we value is, good SAT scores, memorizing how to spell things, a 
good college education, um, and a good, money-making job... and I think that we can preach diversity, and preach that we value all people for what they are, but when it comes back to and what it all boils back down to is that as a society we do not reflect those goals.

Overwhelmingly, cohort members identified and expressed frustration with mixed messages they had seen and experienced in regards to diversity. In fact, the presence of mixed messages appeared to be a vital component of the respondents' understanding of and experience with diversity.

In addition to inconsistent practices, diversity was viewed as a complicated issue, difficult to operationalize and with many facets. Cohort members struggled with how to practice the concept themselves and teach it to others:

(speaker 2)...teachers are in difficult positions as far as that's concerned...diversity's good. I think everybody agrees. We all agree that it's good, but - I mean you know, you come to the point where you've got to make a decision about how much time you spend with the kids that have learning problems; the resources the budget cuts and always a certain amount of students are gonna get....

This difficulty was also expressed in relation to academic differences and the process of grading:

(speaker 1) Tracy can't read, and so-and-so can read, it's like, as a teacher, what do you make of it? When you go to do the report cards like, well, Casey learned five words so, oh my gosh, you know. I'm going to give him the best grade, and then here's some other kid that, without an effort, always messing around in class, but reads a seventh grade book. You just.... when it comes time to explain to the parent, well, this kid got lower than Casey because I, I don't - It's really hard to specialize your expectations for each student. But you feel like you have to, because it's not, 
it's like that five words for Casey makes me want to jump up and down and scream and holler because that other kid that's so high, it's like, I don't feel like that - it was just messing around - and it's true that all the cooperative groups and everything, it feels like it's the right thing to do, but when you get out, you're on your own. You've got to go take a standardized test, usually, to do anything you want to do, you're not gonna be able to pair up with someone and say, well, this is my strength, what's yours? (laughter) Let's put them together. It doesn't work like that. I like having kids work together, I think they learn a lot from it, but I also think that when you get out there, I have needs. That's all. I don't WANT it to be like that, I don't think it should be, but it is.

When speaking of trying to teach the concept diversity in the classroom, on preservice teacher stated, (speaker 4) "I'm trying to figure out. I - trying to help those children understand, you know, that all this society - I mean this is what we all say - we try to say that - but are we really, really sending them the message...".

Mixed messages regarding the concept diversity were also presented by cohort members, themselves, during the focus groups. While the general tone of the discussion indicated a positive value dimension for diversity, including many overt statements to that effect, there were occasions where cohort members indicated otherwise. In one case a cohort member indicated a desire to have a classroom with individuals at all one level:

(speaker 3)lt's just - it kind of blew me away at first, the level at which - I kind of hoped that the level at sixth grade, that everyone would be at the same level, but it's not true. There's just such a big span. 
In response to the discussion regarding the use of standardized tests one individual stated, (speaker 6) "I want them to be able to be successful, be able to get a job. If we don't push them, to meet these standards then where are they? ".

\section{Teachable}

The last major theme the researcher identified from the focused group discussions was diversity as a teachable concept, teachable through various methods. Cohort members spoke often of methods for teaching diversity, almost as if it was tacit knowledge that diversity could be taught in the classroom:

(speaker 8) Well, we just talked about a little bit of diversity in the classroom and it had to do with a speaker coming in and talking about Reparian zones and the environment, and how, if you have, um, along a stream, just, um. like, all ivy, you know you need the diversity to bring in different animals, and different birds, different foods and nutrients, and some could help filter out pollutants and some could help, um, keep the soil and ground, and then we brought that into the classroom, and why is diversity important. And so we were talking about even the male and the female, and the different grades, and then, um, just talking how everybody has something unique to offer and just the variety, and uniqueness, and, um, different resources.

Teachers spoke of teaching diversity through, cultural fairs, holidays and events. One portion of the group discussion centered around a cultural fair as a method for teaching students about the diversity in their school:

(speaker 7)Our school, um, just had a really neat event called a cultural fair, um, the eighth graders, it's a school wide project that all the eighth graders had to do. And basically it's researching 
their family history and tying that to themselves....all the kids made these wonderful displays and it was amazing walking through the gyms because there was such diversity - I mean, there were people from all over the place. And it was, it was a celebration of it - no one was being put down for being this, or put down for being this. I mean it really was to me, it was just fascinating. And some of them had brought out samples of foods from, um they weren't to eat, just for show, different countries; others had picture books. I mean, you know, there were kids from Vietnam, obviously, um, Armenia, um, a, Russia, Ethiopia, um - I mean it just - It was endless - I had no idea that we had so many people from so many different places at (school indicated).

In response one individual stated her experiences with the event:

(speaker 9)! liked the effect it had on the kids when we took them there. Because I think at first they thought "Oh we're just going to get out of class to see this.", but actually when they walked through, there were just rows and rows of all these... awards...they had clothes, food, I mean, jewelry and pictures, and everything, and it was so amazing to them because they walked in and they were like "wow!"' I mean it wasn't what they were expecting, and I think it really affected them.

Cohort members spoke of holidays as events for teaching the concept diversity to students as well, (speaker 10) "When I think of diversity I think of looking at different cultures and accepting teaching the kids to do that and having an activity like this is Black History month, so we incorporate some of that in the program, or it's Chinese New Year, have something going on for that, or Tet, which is new year for Vietnam.". Clearly, while cohort members did not speak of the ability to teach or not to teach diversity, they were able to identify many possible approaches to teaching diversity in their classrooms. 
From the discussion also emerged the ongoing nature of teaching

diversity:

(speaker 1) And to me too, and it sounds great, and I've been in classrooms where, you know, they just did one big celebration, but I think it should be ongoing too, and my mentor teacher has done a lot to show me about that, you know, um, she just does, um, she just does all kinds of stuff. So, I don't know how that... but it's the way you would do it is, uh, is include it in the classroom instead of just having different months...I've been in classrooms where they just, you know, just do one unit on it and that's it, and ok we're done with that, move on to the rest...It should be a part of the everyday curriculum.

When speaking of teaching French in the classroom, one cohort member indicated the practice should happen daily, (speaker 8) "um we could maybe learn some vocabulary and they brainstormed a list of vocab that they would want to learn, and we incorporated it into our everyday, um, counts, our calendar.... So hopefully that can be carried through.".

The issue of community was highly correlated with teaching diversity. Many of the methods suggested for teaching diversity and multiculturalism involved community on some level, whether it be the classroom community, school wide community or the local community. One pre-service teacher spoke of utilizing the experiences of students in the classroom to teach about diversity:

(speaker 1) ....we had a student go to Mexico for a while with family and then come back with stuff, so we talked about where he was going and what he did while he was there. We had a young child visit, she was from the Philippines. And we talked about - and so we, we tried to also make, um, it real to the kids. You - It's like this student in your class and she's from the Philippines...t's little increments throughout the year. 
This comment was echoed by other teachers who indicated their desire to utilize classroom students' experiences to teach about diversity:

(speaker 10) Especially in our school, Vietnamese program, we have a large Vietnamese program there so we're trying to work with some of our students doing an activity, 'cause we've got three Vietnamese students in our class they're second and third graders, so, the um, we're doing some reading, literature, opening their eyes, getting certain kids ideas who haven't seen this stuff before, or know about a certain culture....

The cultural fair was exposed as a situation wherein the differences within the school wide community were used to demonstrate and teach about the concept diversity as well, (speaker 7) "I had no idea that we had so many people from so many different places at (school indicated).".

The pre-service teachers in the focused group interviews, generally, provided many examples of specific teaching strategies designed to assist children in learning about the concept "diversity." One individual recalled a strategy from her student teaching:

(speaker 1) My teacher does this really neat - she has this bulletin that says. "Spotlight's on You", and once a week a different child has to bring something from home, pictures or whatever that they bring - ...the kids, asks questions about themselves, and oh, they talk about their parents, and their little brothers, and their dog that died, and it's just, it's wonderful they just talk about everything.

In this way, children learn about the diversity within their midst from other children. Similarly, another individual spoke of including a language in the classroom curriculum, (speaker 8) "Here's one more, maybe, cultural diverse idea. Um, I brought in a, um, another language in the classroom.". Another 
individual agreed and added still another idea, (speaker 7) "We do that with our deaf students and... they get up and....teach a few things to the kids in the classroom, um, on how to sign certain things, and, um, which gives them the spotlight that they don't always get.".

\section{In-Depth Interviews}

The in-depth interviews, as analyzed by the researcher, yielded a variety of themes as in the focused group discussions, yet the information provided was more detailed and narrative. Respondents in the interviews echoed and confirmed the major themes presented in the focus groups and introduced several new themes as well (see APPENDIX J).

\section{Structures}

The first theme to be echoed by the interview respondents is the concept of diversity as structures. Respondents identified teaching / learning styles, ongoing instruction, instruction through the community, cooperation and competition and other structures as issues relevant to the concept diversity.

Teaching / learning styles. Teaching / learning styles appeared to be a major component of diversity for the respondents. Flexible teaching methods were discussed by all three respondents as key to diversity. Barbara made the point clearly when talking about testing methods used in the classroom:

What I learned through student teaching yet, and and through my own education that - I mean you give a kid a standardized 
test - I mean they might be brilliant but they don't do well on the standardized test because they don't learn things through reading and writing. You know, they need to show you things or explain them verbally. Um. In the mathematics class I taught there was this kid who was a great speaker. I mean he knew everything about anything you ask him he had so much to tell you but when it came to writing it down on the test, he couldn't do it. He was getting F's and D's, you know, because he can't communicate through writing and that's the main type of communication that is used in schools and it's like well, you know, and then he happened to write this, you know, paper on science. It's whatever topic we're studying in science, you know, it's like you get like three lines. And he's like "Well I don't know any more.". And it's like yeah, you do. I know you know a lot more because you told me about it. And so we went to testing him orally. And so it's like, 'cause you know he has the knowledge in his head but he just can't get it out on paper. And also have students who can write so well that you can ask them a question they can't answer it because they don't know how to to process it in their head and come out with an intelligent answer. It comes out like 'uhhh'. But they do know. You just have to find out how they can express it, and so, um, I guess it's another way of seeing diversity in your classroom. So...it's interesting and some through doing things so kids'll learn through like hearing... or a lecture. Some kids'll learn by reading a book [CLEARS THROAT] and some kids learn by all of them and some kids don't learn by any of them.

Chad provided a similar sentiment, highlighting a teacher's necessity for

flexibility of teaching methods. Again teaching and learning styles appear to comprise a component of the concept diversity:

And then she has this whole set of technical things that she does with the kids. Lessons and, um, I'm using her for research, you know things that are good. So they can develop - You can have activities, especially activities that help you too, you know, it's like, you know, flexible. Flexibility. You have to be tremendously flexible too, you know, and so it's hard. 
Specifically, the use of languages as a varied teaching method and structural element of diversity was stressed by Alison. Alison spoke of the importance of languages in attempting to recognize the diversity in the classroom and larger community. She recalled experiences from her student teaching:

But I think I would have brought more into my own classroom, kind of talked, learning a couple things in their language, you know - or at least talking about how is this done differently in the Russian culture, in the Hispanic culture - there's huge differences just - and holidays, and everything - family, and she didn't really go into that at all. I would have played that up more if it was my classroom. I mean, there was - how many kids were there? There was probably four Hispanic, that they spoke Spanish as their first language at home, then there was two or three who spoke different Asian languages... I think it was for grandparents day, and they had the ESL kids up on the stage when I happened to be walking by there was, I think the Hispanic group, and they were speaking in Spanish even though most of the kids do speak English, too. But they were up there speaking in Spanish because their grandparents, many of their grandparents didn't speak English. I'm not sure what else they did, but they definitely brought out a cultural appreciation.... They were singing and doing sign language, doing songs in sign language, so l'd want to do that, too. Because you can at least - I remember when I was in elementary school, we took French and German and um, I still remember songs that we sang, even though I don't really know what they meant - but at least you have that with you - there's other languages out there.

Alison also advocated the use of special holidays and events as a different method to help teach the concept diversity:

We talked about Martin Luther King, and certain holidays. It means, um, everything that you teach, no matter what it is, you know, if you're having a theme, if it's Christmas time, for example, 
then don't just talk about Christmas, talk about all the other, not just Hanukkah or Christmas.

The use of holidays as a method for teaching about diversity was echoed by

Barbara as well, "Um. I've seen a lot of schools you know they do something for African-American history month -".

In addition to holidays and language as teaching tools, Chad spoke of specific teacher behaviors that are connected with teaching and learning styles and diversity:

She has this real good slow body language, and she talks really, you know, clearly. Not - I mean I almost want to say slow but it's not - I mean I don't - that connotes that you're dah or something but not - You know she doesn't, you know, she speaks to the kids, and it's almost kind of a relaxing tone. Um. Another thing she does that l've noticed was that she doesn't appear to be, ah, even when things are out of control or whatever, she doesn't get rattled. She has this calmness. She appeared to be calm regardless of - and that - that's one of the things, um, um, she gets around to all the kids. She makes an effort to be around all the kids, even though she might not right away....And, I mean, although that's a very important component, you know, I think that, ah, there's - there's - there's not no right way to - to inform people or to teach people.

The use of a variety of teaching methods in an effort to accommodate and be aware of a variety of learning styles was often correlated with the concept diversity.

Ongoing. The importance of an ongoing structure for teaching about the concept diversity was raised by all three respondents. In fact, each interviewee felt an ongoing structure was vital to the concept. Barbara stated: 
...but I mean it's just like it's only for a certain amount of time that you learn these specific things about different cultures, and, ah, it seems to me that it should be more incorporated throughout the year, something that you just don't do for a week or you don't do for, you know, a couple days....And, um, we did a lot through you know like reading different books about different cultures, you know, cultures and we studied South America for quite a while....And, um, I mean there are certain units that you have to do in a certain amount of time but, I mean, you shouldn't just like pick out a week and say, "Okay, this week we're going to study Asian people." You know I mean you just can't do that. You have to do more of an encompassing kind of thing. And it has to be something that's done all the time throughout the year. I mean you have to incorporate it with your classroom and keep it going. It's not just something you mention once and then never say again.

Chad spoke of the importance of ongoing structures by stating, "Eh, yeah. I mean I think you'd have to. That's the only way you can teach it....It's definitely has to be something that's ongoing. You know because, ah, you know, or else it doesn't function.". Generally, an ongoing structural element for teaching about diversity appeared to be a primary component to understanding the concept.

Community. Establishing a structure whereby diversity could be taught and addressed with the assistance of or through the use of the community was a theme often raised throughout the interviews. While not often stated explicitly, many examples of methods designed to instruct regarding diversity involve the use of community on some level, either the classroom community, school community or local community. Chad raised the issue clearly:

I mean there's a lot of difference in diversity. It depends where you're teaching that. Um. Some communities will definitely be 
more receptive to the idea, you know, and - and - the community, the position of the community has a lot to determine, or depending on where this school is that determines the amount of influence the community has on the school.

While Chad's example clearly raises the issue of community structures and diversity, other respondents implied a connection by providing examples of teaching strategies that include the community on some level. Alison spoke of the children in her classroom:

...they had and ESL specialist - they've gotta go out and have a class with her, so that was great. But I think I would have brought more into my own classroom, kind of talked, learning a couple things in their language, you know - or at least talking about how is this done differently in the Russian culture, in the Hispanic culture - there's huge differences just - and holidays, and everything - family, and she didn't really go into that at all. I would have played that up more if it was my classroom. I mean, there was - how many kids were there? There was probably four Hispanic, that they spoke Spanish as their first language at home, then there was two or three who spoke different Asian languages, and then there was um, three ethnic Russians...we had ... just assemblies I'm thinking of but we had - I didn't get to see this assembly, but I just walked by and saw a little part of it where - I think it was for grandparents day, and they had the ESL kids up on the stage when I happened to be walking by there was, I think the Hispanic group, and they were speaking in Spanish even though most of the kids do speak English, too. But they were up there speaking in Spanish because their grandparents, many of their grandparents didn't speak English. I'm not sure what else they did, but they definitely brought out a cultural appreciation.

Barbara also indicated the importance of a structure including the community when she spoke of students in her school assisting new students with school policies and practices:

...we had some kids beginning in a year who, um, couldn't speak any English. They were from China and Vietnam, you know, 
and they - just they were right out of - they just came here and went into the public schools because there wasn't room in the ESL class...incoming classes, you know, they didn't speak English at all, and, um, we had some kids that were interested in helping them. You know I mean like: Okay, where are you from and what language do you speak and, you know, and it really helped them a lot, you know, I mean like: Okay, well what you need to do is you need to - they tried to help them with their nametags and stuff like that, you know, because they didn't know what to do. And so... and, um, just helping them with like opening their locker and stuff like that and we had some kids that were really interested in where they were from. You know where are you from? Why did you leave? Why did you come here? You know, um, what kind of things do you do in your country and, I mean kids that were really interested in other cultures and thought that was really cool.

Community as a vehicle for learning about diversity played an important role in cohort members' understanding and teaching about the concept diversity.

Cooperation / competition. Cooperation and competition were raised as structural elements related to diversity in the interviews, however, not as strongly as during the focused group discussion. Barbara raised the importance of a cooperative structure for teaching about and promoting diversity:

...because you have these kids that have the drive and they're competitive and they're always - they want to be better than everybody else and it doesn't really have good classroom dynamics like that. You know -so...cooperation....and stuff like that. We try to focus on that. We try to put them with different groups and different partners just so they would get used to the people in their class and get used to working with different people. 
Chad's opinion was similarly stated, "Diversity is a - is to me the way I perceive it is and that's what part of the cooperation, I mean, because to - my perception of diversity is that everybody are valued equally. I - and so everybody would have a part to play.". While not as explicitly linked to diversity, cooperation, as a structure, still appears to be related to the concept diversity in a positive manner while competition may be negatively related.

Chad raised the issue of general structures in society as they relate to diversity as well. He relayed some of his personal experiences regarding societal structures:

Maybe because th - there's reasons why there's not diversity because people that are prejudice have - had a holding kind of power, um, tend to, ah, you know, screen out, you know, because they don't feel like, you know., they - they already know what they need. What's good for them... That's the whole problem with - with - with a - with the institution of education. That's a - that's a whole problem with the - all messages is to, ah, assume that everything is okay when everything isn't okay. Ah, to assume that everybody started out equal and in fact everybody did not start out equal.... Yeah, ok, you can have, um, the managers whom are white looking for an entry level manager overlook one of the minority persons maybe just because of a prejudice or a stereotype they might have. That contributes to the lack of diversity. I mean that's - and that happens all the time. I mean these guys can be totally equal this black guy and this whatever minority and this white person, and a lot of times it's just that this guy is buddy buddy to the good-ole-boy system.

These general societal structures also play a role in the cohort members' definitions of diversity 
As in the focus groups, the interviewees spoke of different types of diversity or criteria that might be met to constitute diversity. Among these types or criteria are differences, experiences, culture, language, race, "not prejudice", equity and others.

Difference. Difference as a theme played a major role in the interviews conducted with the three respondents. Often the criteria of difference was employed to describe diversity:

...if they don't have an open mind, about diversity, I don't know if they're going to - just try and expose yourself to as many ways of teaching and as many different cultural experiences as you can. Really look in the newspaper and go to different kinds of things that you don't normally do. Because you don't know most of this stuff - it's important....guess when I went in, I went in thinking, ok, different, like, races, different cultures....In a diverse classroom, I see obviously kids of different cultural backgrounds I see kids with their parents so - social backgrounds, different economic backgrounds, different religious backgrounds, um, did - where they come from different regions of the country.

In this case difference identifies the central characteristic of diversity. Chad, perhaps, stated the importance of difference most clearly when he said, "I think it's more important to - for them to try to, ah, make some sincere efforts at, you know, being conscious of differences and how that can effect their teaching practices.".

Experiences. A second major criteria or type of diversity discussed was diversity as different experiences. While this theme emerged from the focused group discussion, it was described in more detail through the 
interviewing process. When speaking of a diverse classroom, one informant stated the importance and impact of experiences:

You know - you know and, um, and then we have kids that travel all over the place and kids that have been to Europe and traveled a lot with their parents and that are, I mean, they're different. I mean they have a different way of thinking. I mean they know much more because they've done that. You know and there's kids who haven't been outside their neighborhood ever.... You know I mean they don't go on vacation with their family. They don't even go to the beach, you know, they just and um, I mean just had experience like that to go to different places, even if you go to the beach, you go to southern Oregon. I mean you're going to learn doing things.

Additionally, experiences were considered necessary to understand or learn about diversity. Respondents, generally, felt diversity could be best, or perhaps only, learned through personal experience rather than through the advice or experience of others. When discussing advice regarding diversity he might give to a student entering a teacher training program Chad stated, "I could not tell them tell them, you know, any advice, aside from giving them practice.".

Culture. Culture was a major criteria or type of diversity discussed during the interviews. While rarely expanded upon, the criteria was often mentioned as central or important to diversity. One individual stated, "I thought of diversity as more of a culture thing.". Another individual added to the response:

I mean in that respect but when I use diversity I'm mostly talking about cultural diversity and, ah, that type of thing and economic diversity, but mostly cultural diversity issues are what I am 
concerned about because they imply a lot of other things. A lot of other issues like economic diversity, racial diversity, etc.

When speaking of how one would teach the concept diversity Alison indicated her ideas about culture as a criteria:

I would still hope that I would bring in other cultures and other - I mean, even if I didn't have an actual human being from another culture, at least, I don't know - instead of just having that one experience...just whatever the kind of subject is, to try and bring in other ways that different cultures use. OK, my work sample was on plants, and I didn't actually do this in my work sample, but I said it was an extension that I could do this to talk about ways that other cultures use plants.

This particular section highlights both the use of experiences and culture to teach diversity.

Language. Language appeared to play a large role in the establishment of diversity for Alison and Barbara. Both Alison and Barbara spoke of language often when describing the diversity of their classrooms. Alison stated, "I had Russian, and Hispanic and English language speaking, so that was, you know - diversity, too". Later she included a suggestion based on her student teaching experience:

I would have - liked to have seen it play in more where. I think my teacher kind of let the ESL - my mentor teacher - kind of let the ESL teacher, 'cause they had an ESL specialist - they gotta go out and have a class with her, so that was great. But I think I would have brought more into my own classroom, kind of talked, learning a couple things in their language, you know.

And when speaking of how one might go about teaching the concept diversity she provided the following example: 
Um, let me think - um, we had .. just assemblies I'm thinking of but we had - I didn't get to see this assembly, but I just walked by and saw a little part of it where - I think it was for grandparents day, and they had the ESL kids up on the stage when I happened to be walking by there was, I think the Hispanic group, and they were speaking in Spanish even though most of the kids do speak English, too. But they were up there speaking in Spanish because their grandparents, many of their grandparents didn't speak English.. I'm not sure what else they did, but they definitely brought out a cultural appreciation.

Barbara stressed a similar importance for language:

You know that was it. We had some kids that were but we had some kids beginning in a year who, um, couldn't speak any English. They were from China and Vietnam, you know, and they - just they were right out of - they just came here and went into the public schools because there wasn't room in the ESL class....incoming classes, you know, they didn't speak English at all, and, um, we had some kids that were interested in helping them. You know I mean like: Okay, where are you from and what language do you speak and, you know, and it really helped them a lot, you know, I mean like: Okay, well what you need to do is you need to - they tried to help them with their nametags and stuff like that, you know, because they didn't know what to do. And so...and, um, just helping them with like opening their locker and stuff like that and we had some kids that were really interested in where they were from. You know where are you from? Why did you leave? Why did you come here? You know, um, what kind of things do you do in your country and, I mean kids that were really interested in other cultures and thought that was really cool.

In this particular situation, students helping other students with language

differences was used to teach about the diversity in the school community.

Race. Race as a criteria for diversity played a prominent role in all three interviews. In fact, race appeared to be a primary criterion for all respondents. Alison stated, "... when I - initially think of diversity I just 
automatically think of race.". This sentiment was echoed by Barbara as well, "I guess when I went in, I went in thinking, ok, different, like, races, different cultures - and that was it.". Chad, while not explicitly identifying race as a criteria, provided many examples of structural issues surrounding diversity and race.

Lack of prejudice. A new criteria that was introduced during the interviews and was touched on by Barbara and Chad is diversity as a lack of prejudice. Each defined diversity at times as the lack of prejudicial actions:

I mean any type of racism that's been carried down through the generations, if you give it to your kids, that's what they're going to know and they'll teach it to their kids and it's just going to keep going and until you wipe it out completely, um, I think there's always going to be mixed messages going on in school.

When speaking of his definition of diversity Chad stated, "there's a lot of prejudices, ah, opinions that people - people have about other people and that's probably the biggest ...problems of - of - of that why we have there's a lack of diversity - all kinds of diversity -".

Equity. An additional new type of diversity introduced during the indepth interviews is the concept of equity as diversity. Both Barbara and Chad spoke of diversity as equity and equal opportunities for people. Barbara stated, "diversity is good and you need to treat people equally.", highlighting equity. Chad defined diversity, "Yeah, diversity, you know, is a - is a - is People understanding that, um, if - there's being equal opportunity...my 
perception of diversity is that everybody are valued equally." Additionally he stated, "That's a - that's a whole problem with the - all messages is to, ah, assume that everything is okay when everything isn't okay. Ah, to assume that everybody started out equal and in fact everybody did not start out equal." Here Chad identifies a common conceptualization of diversity, equal treatment.

Economic. Though not a large theme throughout the interviews, the topic of economics as a criteria for diversity did emerge. Two of the interviewees, Alison and Chad mentioned the topic briefly on a couple of occasions:

(Alison) diversity, too, but also a big thing was economic/class, ...because l'm, you know, kind of middle class, normal university student, I guess, and that was really, really poor school - nothing like any of the schools I went to growing up, you know - I went to public schools, but you know, a wealthier neighborhood. (Chad)...use diversity I'm mostly talking about cultural diversity and, ah, that type of thing and economic diversity.... You know you have to - obviously that's a - a political/economic issue.

Both of these individuals mentioned economics only briefly but certainly highlighted its importance for the concept diversity.

Other themes mentioned briefly during the interviews include: diversity as "always there", diversity as something one "can't teach".

\section{Mixed Messages}


A major part of the focused group discussions, mixed messages as a theme related to diversity resurfaced during all three interviews. Respondents spoke of diversity as connected to mixed messages in their experience, and also spoke of diversity as a complicated concept. Interviewees occasionally provided mixed messages in their own accounts as well, including a number of incidents where individuals indicated race was not an issue in the classroom.

Mixed messages. Alison spoke of mixed messages and diversity as she saw it in the classroom through her experiences. In her experiences, these mixed messages came from both students and teachers alike:

We talked about Martin Luther King, and certain holidays, but most of the books we were reading, I don't think she made a real effort to put a lot of different books with different races in it - there were more animal books... One time the teacher was reading the Scholastic newspaper and there was an African-American woman who was a police chief that she was talking about. She was making more of a point of her being a woman, but she also said she's African-American, anybody can be a police chief, or whatever. Actually the teacher said, "black," and the little body raise his hand and said, "African-American." She just went, "Oh yeah, sorry."

Chad's experiences spoke to the idea of mixed messages in his experiences as well. Generally Chad's notions of mixed messages come from his experiences as a student rather than as a student teacher:

That's a - that's a whole problem with the - all messages is to, ah, assume that everything is okay when everything isn't okay. Ah, to assume that everybody started out equal and in fact everybody did not start out equal... Yeah, ok, you can have, um, the managers whom are white looking for an entry level 
manager overlook one of the minority persons maybe just because of a prejudice or a stereotype they might have. That contributes to the lack of diversity. I mean that's - and that happens all the time. I mean these guys can be totally equal this black guy and this whatever minority and this white person, and a lot of times it's just that this guy is buddy buddy to the good-ole-boy system.

Brenda spoke of similar mixed messages in classrooms she experienced. In this case the messages came from a student and were unmistakable.

Additionally, Brenda highlights the mixed messages regarding diversity that can exist between the home and the classroom:

I mean the class was predominately, you know, um, white. And, ah, he gets along with these kids really well but, you know, sitting there talking to his friend he's like "I went to the Lloyd Center the other day. And I don't like going there 'cause all you see are black people." And I was just like "uhh, I can't believe you said that?" [LAUGHTER] because everybody heard him. And it's like, ok, these kids, they're just going to go ballistic. But nobody said anything. But I mean, you know, we just got done with Black American history month and he said that.

She summed it up by saying, "Definitely a mixed message. Um. I don't know I mean in school, you know, teachers...'You know everybody's equal' and all this kind of stuff, and, I mean, they get it at school but a lot of kids don't get it at home.".

Complicated. It appeared that mixed messages were also experienced as complications within the concept diversity for the interviewees. After discussing the incident where she overheard her student make a derogatory 
remark about black people at Lloyd Centre Brenda commented about the situation:

Yeah, and so - I mean - I don't - I really don't think he understood what he said - So, and I don't think, I mean he's not I don't know how it can, you know, like you know, it - it kind of offended me, you know, that he even [LAUGHTER] actually said that, and so, um, I don't know of any way sort of really to say - to let him know that it was offensive because he's not in a situation where he would know it is and so -it was just - I was surprised that that came out of his mouth.

Later when speaking of the issue of race in the classroom she stated:

You know only now that they...I mean I know they notice but they just don't seem to - It doesn't matter, I guess, which in a way it's kind of good but in a way it's not because there's just kind of - they don't care so - in a way it kind of shows a lack of education on their part but in a way it kind of accepting too so It - I think I need spend more time in the classroom too. See how that will work out.

Chad also spoke of the difficulty and complication of attempting to develop consistency between messages provided at home and at school:

...it's harder for kids to make that distinction so, you know, you run into trouble when you teach that to kids and especially when you run into the parent/family influence. That makes it - ah, even more difficult for them, the kid to decipher all that information and to try to understand and comprehend some or they might necessarily agree with it or/and that combined with what they're learning at home which is the most important ones.

Again. the complicated nature of the concept diversity appears to be a vital component to understanding and defining it.

Respondents' mixed messages. The respondents themselves occasionally provided mixed messages in regards to the concept diversity. 
Chad stated he thought diversity as a concept could not be taught but later advocated teaching diversity as a critical subject in an ongoing manner. After discussing the boy who spoke of "too many blacks at Lloyd Centre" Brenda was asked, "What role do you think kind of, and that example is really interesting, but what role do you think race played or plays in the classroom that you've been in?." She stated, "Um. It didn't really seem like they really cared what race their friends were. I mean it didn't matter, they were their friends and so -". This statement clearly contradicts the passage Brenda provided earlier where a student commented on the "blacks at Lloyd Centre."

Alison was asked a similar question by the researcher:

one of the things you said in the very beginning was that when people think about diversity, race is one of the first things that people think of, but in our group discussion, it wasn't talked about very much; it was kind of mentioned and people said one of the things they talked about was race. I was wondering, then, based on your experience, if you have any ideas of how race fits into the classroom? Based on what you were talking about, you said you didn't have very much of a racial mix; but did you find it playing up at all in your classroom?

Her response indicated race was not an issue:

No, not really; we just had one African-American student, that was it. And um, I don't know; we didn't do - my mentor teacher was Asian-American, and she - she didn't really - I don't know - make an issue of it all. We talked about Martin Luther King, and certain holidays, but most of the books we were reading, I don't think she made a real effort to put a lot of different books with different races in it - there were more animal books - um, No, it didn't play out; nobody ever said anything about him, nobody ever said anything about the Asian students at all. It really wasn't an issue at all, and I don't know if that's because of the age either - it might be -It wasn't an issue at all $-I$ don't know; I mean, I think it might 
be different if there was more of a cross-section of different kinds of different races, um - I don't know, you know, if there was another African-American student, would they have hung out together? You know? I have no idea -...Yeah - I mean, I think it's good - the boy, he was - he got along with people, but he was also, he was really hyper, so the students he hung out with were kind of more hyper, too - troublemakers! So I don't know it didn't really go one way or the other - One time the teacher was reading the Scholastic newspaper and there was an AfricanAmerican woman who was a police chief that she was talking about. She was making more of a point of her being a woman, but she also said she's African-American, anybody can be a police chief, or whatever. Actually the teacher said, "black", and the little body raise his hand and said, "African-American." She just went, "Oh yeah, sorry". So I thought that was cute.

In this situation, Alison provided mixed messages by indicating race was not an issue and then proceeding to provide a number of instances where race was a vital part of the classroom environment.

\section{Teachable}

Diversity as a teachable concept, or at least appreciation for or awareness of diversity as teachable concepts, was an identified assumption during the focused group discussions that the researcher attempted to clarify during the in-depth interviews. During the interviews, respondents, indeed confirmed the teachable nature of appreciation for and awareness of diversity as a concept. Diversity, appreciation for diversity and awareness of diversity were addressed as teachable concepts, teachable through varied teaching and learning styles, ongoing structures, and the use of the community (all structural elements identified in the aforementioned section on diversity as 
defined structurally). Respondents also indicated diversity could be taught through experiences.

Teaching style / learning style. Varied teaching and learning styles played a key role in the discussion of diversity as a teachable concept. Speaking of how one might bring diversity into the classroom, Alison suggested:

Um, let me think - um, we had .. just assemblies I'm thinking of but we had - I didn't get to see this assembly, but I just walked by and saw a little part of it where - I think it was for grandparents day, and they had the ESL kids up on the stage when I happened to be walking by there was, I think the Hispanic group, and they were speaking in Spanish even though most of the kids do speak English, too. But they were up there speaking in Spanish because their grandparents, many of their grandparents didn't speak English.. I'm not sure what else they did, but they definitely brought out a cultural appreciation in that - um, how else? Just exposure - plays, music.

Brenda added a similar sentiment regarding varied teaching methods and learning styles as a way to teach the concept diversity:

What I learned through student teaching yet, and and through my own education that - I mean you give a kid a standardized test - I mean they might be brilliant but they don't do well on the standardized test because they don't learn things through reading and writing. You know, they need to show you things or explain them verbally. Um. In the mathematics class I taught there was this kid who was a great speaker. I mean he knew everything about anything you ask him he had so much to tell you but when it came to writing it down on the test, he couldn't do it. He was getting F's and D's, you know, because he can't communicate through writing and that's the main type of communication that is used in schools and it's like well, you know, and then he happened to write this, you know, paper on science. It's whatever topic we're studying in science, you know, it's like you get like three lines. And he's like "Well I don't know 
any more.". And it's like yeah, you do. I know you know a lot more because you told me about it. And so we went to testing him orally. And so it's like, 'cause you know he has the knowledge in his head but he just can't get it out on paper. And also have students who can write so well that you can ask them a question they can't answer it because they don't know how to to process it in their head and come out with an intelligent answer. It comes out like "uhhh". But they do know. You just have to find out how they can express it, and so, um, I guess it's another way of seeing diversity in your classroom.

In these situations cohort members spoke of their own learning about diversity through studying about different teaching and learning styles. Additionally, respondents identified, though not explicitly, the importance of teaching about diversity through demonstration of the concept, varied teaching styles.

Ongoing. An ongoing structure proved to be vital to the teaching of diversity as a concept in the opinion of all the respondents. Each individual addressed this point during his or her interview:

(Alison) Ongoing teaching diversity? I don't - yeah, I think it teaches diversity and it teaches tolerance, too. It's not just saying that the one way, there's one way and everyone else does it wrong, or everyone does it the same way. Just so they know that other things are out there - so MANY other things are out there! I'm not telling them everything there is out there... Kind of just do every day, trying to do it all the time - put a phrase in here or there, or really put a concentrated effort into extending the unit. Show other cultures.

Barbara shared a particularly clear example of the concept of teaching diversity through ongoing methods:

I mean it's just like it's only for a certain amount of time that you learn these specific things about different cultures, and, ah, it seems to me that it should be more incorporated throughout the year, something that you just don't do for a week or you don't do 
for, you know, a couple days.... I mean, you shouldn't just like pick out a week and say, "Okay, this week we're going to study Asian people.". You know I mean you just can't do that. You have to do more of an encompassing kind of thing. And it has to be something that's done all the time throughout the year. You just can't let..., and then talk about it again. I mean you have to incorporate it with your classroom and keep it going. It's not just something you mention once and then never say again.

When asked "So what happens do you think if - or if you only do it for a week

or what is the drawback of only doing it for a week?", Barbara responded:

Um. I think it - I don't think kids appreciate diversity - that much. I mean it's like: Okay, for a week we're going to appreciate these people, and then you - you don't ever mention it again, and so, um, every kid in the class and we had like only about three African-American students in our class. I mean the class was predominately, you know, um, white. And, ah, he gets along with these kids really well but, you know, sitting there talking to his friend he's like "I went to the Lloyd Center the other day. And I don't like going there 'cause all you see are black people". And I was just like "uhh, I can't believe you said that?" [LAUGHTER] because everybody heard him. And it's like, ok, these kids, they're just going to go ballistic. But nobody said anything. But I mean, you know, we just got done with Black American history month and he said that.

Barbara's example provides a clear look into the cohort members beliefs regarding the necessity of ongoing instruction to teach diversity.

Community. The community appears to play a large role in teaching about diversity and was mentioned often by respondents. Alison spoke of including the grandparents of school children when teaching diversity through language:

Um, let me think - um, we had .. just assemblies I'm thinking of but we had - I didn't get to see this assembly, but I just walked by and saw a little part of it where - I think it was for grandparents 
day, and they had the ESL kids up on the stage when I happened to be walking by there was, I think the Hispanic group, and they were speaking in Spanish even though most of the kids do speak English, too. But they were up there speaking in Spanish because their grandparents, many of their grandparents didn't speak English. I'm not sure what else they did, but they definitely brought out a cultural appreciation.

Barbara spoke of a similar use of community in the classroom. She recalled children learning about diversity through interaction with their classmates who had come from different countries:

And so...and, um, just helping them with like opening their locker and stuff like that and we had some kids that were really interested in where they were from. You know where are you from? Why did you leave? Why did you come here? You know, um, what kind of things do you do in your country and, I mean kids that were really interested in other cultures and thought that was really cool.

In both Alison and Barbara's examples community plays a large role in the ability to teach students about diversity.

Experiences. Respondents also spoke of learning diversity through experiences. Rather than through other methods, respondents often spoke of educating through experiential learning. To provide advice to a pre-service teacher regarding learning about diversity, Alison stated:

just try and expose yourself to as many ways of teaching and as many different cultural experiences as you can. Really look in the newspaper and go to different kinds of things that you don't normally do. Because you don't know most of this stuff - it's important. 
Chad answered the same question by indicating, "I could not tell them tell

them, you know, any advice, aside from giving them practice." Barbara

suggested a similar importance for experience:

You know I mean they don't go on vacation with their family.

They don't even go to the beach, you know, they just - and um, I mean just had experience like that to go to different places, even if you go to the beach, you go to southern Oregon. I mean you're going to learn doing things.... We try to put them with different groups and different partners just so they would get used to the people in their class and get used to working with different people because, you know, I mean when they go on in school and when they go out, you know, in order to keep their jobs, I mean, your going to have to work with people you don't like. You know - [LAUGHTER] - and you are going to have to work with people that you do like, you know. I mean there's people that slough off and there's people that, you know, are overachievers and, you know, there's just all different kinds of people and you... have to work with them all your life so you better get used to it now, you know, and they come to me (whispers) "I don't want to work with so and so.". And it's like well you need to figure out how to work it out in your group. You know you need to figure this out because you hear - I mean when you go out into the world you're going to have people that don't do anything, you know, and it's going to effect your group probably so you need to figure out how to work in your group to get everything done.

Similar to Barbara's advice for her students, Chad spoke of learning about

diversity himself through his own experiences in the community:

A lot of my philosophy and beliefs are based on, ah, moral principles, internal standards, my experience, and my history, you know. A lot of African communities had communal systems. A lot of them do. Not all of them but a lot of them did have that and, you know, I have really - it does a lot for my psyche - for my for me to know that my ancestors appreciated a system, value system that, um, that co - that appreciated individuals everybody's united effort. 
In Chad's situation he expressed his own understanding of diversity that developed with experience.

\section{New Themes}

As the researcher analyzed the in-depth interviews, at least two new themes emerged from the transcripts. First, respondents spoke of the definition of diversity as ambiguous. Each individual spoke of either uncertainty about a definition or of simply not knowing. Additionally, most often in conjunction with uncertainty, respondents spoke of the definition as something that had changed for them over the course of their education or as something for which there is no single definition. Second, respondents spoke of why one should attempt to teach diversity or awareness of and appreciation for diversity.

Uncertainty. Throughout the interviews, respondents spoke of being uncertain of a definition of diversity, themselves, or of an uncertainty on the part of others. Connected to this sentiment was often the sentiment that a personal definition of diversity had changed or that there was no one definition for the concept. Alison indicated her opinion was unclear:

People were kinda unclear about it; I don't know if it's just us, or Well, um, I mean, it's just kind of , like I said, you know, I wouldn't have initially think race, we think diversity, but in the beginning, in (name) class, we wrote a cross-cultural autobiography, and we were supposed to talk about our life and how we've been affected by other cultures, and he very clearly said after he passed them out, that culture and race aren't the same thing, and I understand that, I mean, you know, I can get that, but - I think it's still part of 
diversity, culture and race, it's just - kind of a foggy area everybody has their own idea about it, but I don't think it's really defined, you know what I mean.

Similarly, Barbara began her interview by offering, "our views have changed but I think they've expanded more so -Yeah. Um, I think diversity kind of changed the meaning, I guess the meaning changed for me during my student teaching.". She went on later to say:

And so you have like your African-American population, ah that's - And I mean it's a narrow point of view but I never thought about it before.....and so for me it's not the thing that I actually sat down and thought about and so, um, when we start talking about diversity, um, I think a lot of people had real narrow scope as to what that meant....And, um, for me it broadened a lot during my student teaching.

Chad clearly indicated his belief there is not one single definition of diversity to be learned, "I mean there's not one definition of diversity and what it means to - that's not even constructive, I don't think.". He went on later to state, "A lot of people don't really know what diversity means....So my def - I mean - to me, you know, of - actually all the cases it means handling the situations and opportunities, um, equity - equity, I guess.".

Why teach diversity? Respondents in the in-depth interviews raised the issue of a reason to teach diversity. During the discussions, each volunteered a reason why diversity should be addressed in the schools without a prompt from the researcher. Alison, when providing advice for a new pre-service teacher offered: 
...just try and expose yourself to as many ways of teaching and as many different cultural experiences as you can. Really look in the newspaper and go to different kinds of things that you don't normally do. Because you don't know most of this stuff - it's important.

Addressing the importance of teaching diversity during her interview, as well,

Barbara indicated:

But I mean the only thing that I think you can do as an educator is to, you know, let people know that, you know, that if you didn't have diversity you would never learn anything. I mean everything would be the same. Everybody would be the same if there wasn't any diversity in your classroom at all. And you wouldn't learn anything new. You'd never experience anything new, and you'd just be so focused on your own society but, you know, it would just make things a lot worse, you know. I don't know. Ha. But I think, you know, in - in school you have to, you know, get the message out that you need diversity in the classroom or in the -in the neighborhood or in a city or, you know.

However, Chad's contributions were, perhaps, most clear when he spoke of why it is important for students and teachers, all individuals to educate themselves regarding diversity:

Yeah, diversity is - is - it's really pertinent because I mean it's [SIGHS] I think it, you know, like the world is shrinking, you know, per se, you know, ah, with communications it really is, and, ah, you know, um, I think that people are in for a rude awakening if they don't realize how to communicate from both groups. Rude awakening meaning-I don't know if this probably negative but maybe positive but just a rude awakening period for both groups-all groups if - if, ah, they don't realize that, you know, we need to learn how to communicate with each other and that is important, you know, but I guess, it just, if they realize it is important then they learn how to communicate.... I make an effort to find out about others, beside from myself, you know, because I don't - I don't think that it's - it's not - it's not 
beneficial for me to just have an understanding of what I am. In other words I'm not just here ... by myself.

Clearly the cohort members interviewed felt the concept to be an important concept for them to learn and for them to bring into the classroom. While the specific reasons or motivations to teach about diversity appear to be unique to each individual the concept was highlighted as critical to all respondents. 
CHAPTER IV

\section{DISCUSSION}

"Diversity" has become a word associated with many professions, institutions, and contexts in US society today. Diversity is paired with words such as standards, consultants, training, awareness and others. However, the researcher focused on how diversity is defined or conceptualized by preservice teachers in the Children and Communities cohort at Portland State University. Diversity is especially important as it relates to educators and preservice teachers. Teachers will, today, be instructing children of different backgrounds and races more than ever in the past (Ross \& Smith, 1992). And teachers conceptualizations and behaviors have profound impacts on student performance and success. Past studies have shown teachers' prior expectations for students impact student outcomes (Ladson-Billings, 1994; Rosenthal \& Babad, 1985). Simply put, "...how teachers...think about education and students makes a pronounced difference in student performance and achievement." (Ladson-Billings, 1994, p. 22). In such situations, the ways teachers understand the concept "diversity" may be vital. However, upon review of much of the education literature addressing 
"diversity" as a concept, the researcher identified many opinion papers and discussions of what should or ought to be included in the concept diversity and taught in schools but few studies that took the initiative to ask teachers, themselves how they understood the concept. Much of the education literature discusses "diversity" apart from teachers' understandings of the concept. Additionally, the debate in the literature is one complicated and often contradictory.

In an effort to come to understand the ways pre-service teachers conceptualize "diversity," a literature search was conducted to identify past and present conceptualizations of diversity within the institution of education and more broadly. In addition, an open-ended survey question was administered, and two focused group discussions and three in-depth interviews were conducted. The survey question and transcripts from both the focused group discussions and in-depth interviews were analyzed in an effort to identify emergent themes or units of meaning.

A theoretical perspective was established in the theory chapter citing Chaffee's (1991) lower-order concepts. Lower-order concepts are said to be smaller units which comprise a larger unit of meaning, the higher-order concept. These lower-order concepts are more closely associated to observable behavior and should be more easily measured than the broader higher-order concept. In this case, the education literature identifies 
language, learning style, teaching style, race, socio-economic status, ability, gender, hobbies, sexual orientation, occupation, religious affiliation, age, difference and democracy (see APPENDIX B) as lower order concepts for the higher order concept "diversity." However, based on the surveys, focused group discussions and in-depth interviews, it appears not all of these lower order concepts are equally relevant for pre-service teachers in the Children and Communities cohort. Cohort members spoke of language, teaching and learning styles, race, socio-economic status, religion, gender and ability as components relevant to their experience (see general findings). Lower order concepts hobbies, sexual orientation, occupation and age were not mentioned in the interviews.

Constructivism, as a theoretical perspective was also established in the theory chapter. According to constructivism, the human is an active agent, shaping his or her own environment, in part through interpretation of events (Delia, 1977). By using constructs, pairs of bi-polar opposites, an individual engages in the process of creating meaning. An individual may identify an event, experience or object and classify it along a number of relevant constructs in order to make sense of that object or experience object in relation to similar phenomena. While this categorization of events etc. along relevant constructs is an individual, interpretive process, the creation of the relevant constructs may, in fact, be a social process (Littlejohn, 1992). 
"Diversity" as a concept can similarly be understood in terms of bipolar constructs. Within the education literature, the constructs, assimilationism / pluralism and ethnocentrism / ethnorelativism have clearly been utilized to make meaning of "diversity." Additionally, evaluative dimensions such as good and bad, active and passive, growing and dying, and comforting and threatening are implied. Among these constructs, ethnocentric behavior was mentioned as a developmental stage on one occasion during the interviews, and diversity was addressed as a positive element on another occasion, the good / bad construct. Additionally, an implicit positive tone toward "diversity" existed throughout the group discussion and interviews, further implicating a good / bad construct. Yet this was rarely an overt topic. Assimilationism / pluralism, active and passive, growing and dying, comforting and threatening were not discussed within the surveys, focus group discussions or interviews.

\section{General Findings}

Upon evaluation of the survey question, focused group discussions and in-depth interviews the researcher identified an abundance of themes respondents appeared to correlate with the concept "diversity" and many complicated and conflicting messages as well (not unlike the literature regarding the topic). Two of the emergent themes suggest additional lower order concepts: "Structures" and "Mixed Messages." The emergent themes 
"Teachable" and "Uncertain" suggest new constructs. And a final theme appears to correlate with the "Cultural" dimension of the education literature:

"Types or Criteria."

\section{Structures}

Included within the lower-order "structures" thematic category are concepts such as "special expectations," "teaching styles / learning styles" and "standards." Also emerging under the category "structures" is the construct "cooperation / competition." Among the most salient contributions from cohort members include a statement regarding structures of assignment parameters:

(speaker 1) I set parameters up, to include the siblings at the top, your parents and your, and you know, grandparents - I had to set parameters, or the kids would, you know - but this one child, to him and in the Asian Community, cousins, and aunts \& uncles are also included in there so his whole tree was covered. And my mentor teacher didn't - I mean she's like "You can't even see his tree." And I thought, well, you know, this is his family tree to him and I have to let him do that, I have to accept that, you know, he needs to go beyond my parameters because, you know - there's two, and, I don't know, I just thought, you're teaching it, but you're not really accepting it yourself too. Even though you set parameters, you have to be willing to be - (speaker 2) Flexible. Flexible. Flexibility, key word. (laughter)

Also included under this theme is a set of statements regarding standardized tests:

(speaker 4) We're doing the same thing; our math scores at our school were low, and so they're basically telling us they want us to teach the kids to the test. They want us to teach them the stuff 
that's gonna be on the test so they can bump those scores up. And that just goes against everything...

(speaker 5) Ya, the heck with all the diversity and all the levels, you have one level - to get the school on a high level for that test.

That's all that counts.

Many of these structural concerns align with the sentiments provided by Nieto (1992). She suggests teachers are products of educational systems that have histories of racism, and unaccommodating pedagogy. As a result of their experiences with the educational system, teachers, themselves, put into practice what they have experienced within the institution of education and perpetuate structures that may be harmful to many of their students. She highlights the opinion of many educators by stating learning styles and pedagogical variation are crucial to the concept "diversity."

\section{Types Of Diversity Or Criteria}

The thematic category "types of diversity or criteria" includes a wide variety of subthemes. Most prominently, the concept "difference" emerged as central to the concept diversity, (speaker 1) "...but diversity there is not diversity. It's not differences - essentially, how I think of diversity, is there are differences." Additionally, academic diversity played a prominent role in the discussion:

(speaker 3) The diversity I see most is the academic - it's the range and level at which kids learn, there's more than one level in the sixth grade class it's like there's such a huge span of, of what grade you're in. There's kids always at the bottom, some kids at the top and just a bunch in the middle. 
Other themes to arise in the discussion of criteria include familiar concepts such as, race, ethnicity, gender, economic, and language, culture, origins, artifacts and mental and physical ability. This thematic category, types of diversity or criteria, appears to correlate with the "cultural diversity" dimension of the education literature. In each case, the category serves as a venue for listing groups which constitute diversity or criteria which entail diversity. However, neither the thematic category emergent from the focused groups nor the literature expound on the criteria.

\section{Mixed Messages}

The lower-order concept theme of "mixed messages" played a significant role during the focused group discussions and in-depth interviews.

Respondents clearly identified areas of inconsistency within the concept diversity as they had experienced it:

(speaker 2) ... understand that there's mixed signals as far as diversity is concerned, you know, saying diversity is good, but yet, that's not really what's happening...So you can say diversity is good, but it's not happening. It's not being practiced. So you're giving mixed signals...Diversity, it's a tough thing. (speaker 1) In a sense, so what you're saying, is the real world doesn't practice what we preach? At all? We can teach them about diversity, and talk about diversity and use diversity in the classroom but when we get outside, it's different.

This dimension, while present in the literature by example (conflicting definitions of diversity such as the assimilationist and pluralist perspectives), plays only a small role in terms of a research topic. Most discussion of mixed messages 
within the education literature consists of opinion papers only, few teachers have been asked about their understanding of these apparent contradictions or how they might be remedied within the institution of education. Yet this dimension constitutes a significant portion of cohort members' understanding of and experiences with the concept diversity. Mixed messages appear to be a central part of what is diversity for these pre-service teachers.

\section{Teachable}

From the focused group discussions and interviews, it appeared diversity was tacitly understood to be a teachable concept, suggesting a new construct "teachable / unteachable." Respondents spoke of how diversity, as a concept, might be taught in the classroom and of specific techniques. Teachers spoke of teaching diversity through cultural fairs, holidays, events, language and by using the classroom and local community. While, certainly a small part of the interview, one respondent briefly wrestled with the assumption that diversity could be taught. Chad vacillated between diversity as teachable and not teachable only to end up providing examples of how one might teach the concept. This theme, again, is identified within the literature but only tacitly and through opinion papers. Many individuals have written about how teachers might go about teaching the concept diversity with the tacit assumption that diversity can be taught in the classroom. Little research has been attempted regarding the feasibility of teaching diversity or the effectiveness of any 
particular pedagogical method of teaching diversity. Yet this appears to be an assumption to be questioned.

\section{Uncertain}

Throughout the interviews, respondents spoke of being uncertain of a definition of diversity, themselves, or of an uncertainty on the part of others. Connected to this sentiment of ambiguity was often the sentiment that a personal definition of diversity had changed or that there was no one definition for the concept. This uncertainty suggests a new construct, "uncertain / certain" or "ambiguous / clear." Alison indicated her opinion was unclear:

People were kinda unclear about it; I don't know if it's just us, or Well, um, I mean, it's just kind of , like I said, you know, I wouldn't have initially think race, we think diversity, but in the beginning, in (name) class, we wrote a cross-cultural autobiography, and we were supposed to talk about our life and how we've been affected by other cultures, and he very clearly said after he passed them out, that culture and race aren't the same thing, and I understand that, I mean, you know, I can get that, but - I think it's still part of diversity, culture and race, it's just - kind of a foggy area everybody has their own idea about it, but I don't think it's really defined, you know what I mean.

Chad clearly indicated his belief there is not one single definition of diversity to be learned, "I mean there's not one definition of diversity and what it means to - that's not even constructive, I don't think.". He went on later to state, "A lot of people don't really know what diversity means." Within the education literature, rarely is there discussion of the ambiguous nature of the meaning of diversity. While there is occasionally a piece on the individual nature of 
meaning, teachers themselves have not been asked to talk about the nature of the concept "diversity" so the literature is lacking an "ambiguous" or "uncertain" response.

\section{General Suggestions}

Selecting open ended survey questions, focused group discussions, and in-depth interviews as research methods has implications for the findings of any study. In the case of this particular research, respondents felt they were constrained by group dynamics yet aided in generating new ideas by other cohort members during the group discussion:

(Alison) ...when there's a group, sometimes people tend to dominate the conversation and not everybody says what they're thinking or what they're feeling...I think that there was a couple of people that kind of talked a lot more, and they usually do talk a lot more than - you know, it's just like in any other class that um, by now we all know how to deal with that. (Alison) ...I think the group's good because people say things and it's kind of like a chain reaction, they start stirring up people - I think they're both good, I think that you should have both, because um, the interview, obviously, l've been able to go more in-depth.

Generally, the research method appears to suit the research question well, providing checks and balances. Respondents have the opportunity to hear one another in the group discussion and piggyback off one another's ideas but can discuss sensitive issues in the in-depth interview if they feel unable to disclose in the group discussion. In future research regarding "diversity" the researcher would suggest similar methods. However, for future study, the researcher 
would suggest more in-depth interviews to accompany the focused group discussion. Respondents held back their uncertainty regarding diversity while in the group discussion; yet each interviewee expressed personal uncertainty or uncertainty on the part of others. Whether this was a result of social desirability characteristics or because cohort advisors were in the room, the topic of "diversity as ambiguous" came out only in the in-depth interviews. With a greater opportunity to discuss with respondents would come a greater understanding of their meaning structures and sensitive issues. Additionally, the interviews provide a greater opportunity to clarify issues raised during the focused group discussion format.

\section{Suggestions For Future Research}

The themes generated from the survey question, focused group discussions, and in-depth interviews suggest a need to further explore themes of "mixed messages" and "diversity as ambiguous." Respondents discussed, on many occasions the complicated nature of the concept "diversity" and the complexity involved in operationalizing such a concept. Clearly, further research regarding the nature of operationalization in the classroom is necessary to better understand how teachers plan to operationalize diversity in their classrooms. Additionally, it is interesting to note that although members of the Children and Communities cohort had been involved in training regarding 
the concept diversity, its definitions, and implications for the classroom, each interviewee indicated the definition of the concept to be problematic. Further research is needed to clarify this "diversity as ambiguous" theme. How prevalent is the ambiguous nature of the concept "diversity?" How might one go about clarifying the concept? How prevalent is the "changing" or "expanding" nature of the concept?

Finally, it is clear the voices of teachers in the education literature regarding "diversity" are missing. Much opinion regarding how diversity should be understood and operationalized has been written. Yet little research involving teachers themselves, and their voices, exists. Respondents in the indepth interviews independently raised the issue of a reason to teach diversity. Further research might seek to remedy the missing voices and understand why teachers think it is important to understand "diversity." During the discussions, each volunteered a reason why diversity should be addressed in the schools without a prompt from the researcher. Alison, when providing advice for a new pre-service teacher offered, "expose yourself to as many ways of teaching and as many different cultural experiences as you can. Really look in the newspaper and go to different kinds of things that you don't normally do... you don't know most of this stuff - it's important.". Barbara Addressed the importance of teaching diversity during her interview, as well:

But I mean the only thing that I think you can do as an educator is to, you know, let people know that, you know, that if you didn't have diversity you would never learn anything. I mean 
everything would be the same. Everybody would be the same if there wasn't any diversity in your classroom at all. And you wouldn't learn anything new. You'd never experience anything new, and you'd just be so focused on your own society but, you know, it would just make things a lot worse, you know. I don't know. Ha. But I think, you know, in - in school you have to, you know, get the message out that you need diversity in the classroom or in the -in the neighborhood or in a city or, you know.

For Barbara the importance appears to be the enjoyment and excitement of new experiences. Chad's contributions were, perhaps, most clear:

Yeah, diversity is - is - it's really pertinent because I mean it's [SIGHS] I think it, you know, like the world is shrinking, you know, per se, you know, ah, with communications it really is, and, ah, you know, um, I think that people are in for a rude awakening if they don't realize how to communicate from both groups. Rude awakening meaning-I don't know if this probably negative but maybe positive but just a rude awakening period for both groups-all groups if - if, ah, they don't realize that, you know, we need to learn how to communicate with each other and that is important, you know, but I guess, it just, if they realize it is important then they learn how to communicate... I make an effort to find out about others, beside from myself, you know, because I don't - I don't think that it's - it's not - it's not beneficial for me to just have an understanding of what I am...In other words I'm not just here ... by myself.

These contributions, in the words of the cohort members themselves, provide compelling reason for the importance of understanding the ways pre-service teachers conceptualize diversity.

In short, it appears as if members of the Children and Communities cohort are aware of and are able to articulate the importance of understanding "diversity." Respondents identified personal experiences that aided in the 
development of their own understanding of "diversity" and specific methods one might use to teach others, citing numerous, concrete examples. However, the concept appears to remain problematic both for cohort members and within the education literature. Interviewees indicated their own uncertainty about what was included in the concept and what was not as well as the suspicion that others were also unsure. Additionally, the focused group discussion and indepth interviews highlighted the mixed messages related to the concept. Respondents spoke of trouble knowing how to "really send the message" to their students and occasionally presented mixed messages of their own, contradicting their own statements. Chad's comment, perhaps sums up the issue best:

But, um, I mean I just looked at it in its context for what - what it is, you know, you have teachers that, um, pre-service teachers trying to formulate in their minds, you know, some concepts of how they're going to interact with, ah, students that are minority students and - and I think it's...important to - for them to try to, ah, make some sincere efforts at, you know, being conscious of differences and how that can effect their teaching practices. 


\section{REFERENCES}

Almack, J. C. (1924). Education for Citizenship. Cambridge, MA: The Riverside Press.

Aune, B. (1967). Knowledge, Mind, and Nature. New York: Random House.

Babbie, E. (1995) The Practice of Social Research: (7th Ed.) San Francisco, CA: Wadsworth.

Banks, J. A., (1992). Curriculum guidelines for multicultural education. Social Education, Sept. pp. 274-294.

Banks, J. A., (1994). Transforming the mainstream curriculum, Educational Leadership, 51. pp. 4-8.

Bennett, M. J. (1986). A developmental approach to training for intercultural sensitivity. International Journal of Intercultural Relations, 10. pp. 179196.

Carnoy, M., \& Levin, H. M. (1985). Schooling and Work in the Democratic State. Stanford, CA: Stanford University Press. 
Chaffee, C. H. (1991). Explication. Newburry Park, CA: Sage.

Cheng, L. L. (1990). Recognizing diversity. American Behavioral Scientist, 34. pp. 263-278.

Clarke, R. (1964). The Diversity of Man.New York, NY: Roy Publishers.

Cortes, C. E., (1991). Pluribus \& unum the quest for community amid diversity. Change, Sept./Oct. pp. 8-13.

Crabtree, B. F., \& Miller, W. L. (1992). Doing Qualitative Research. Newbury Park, CA: Sage.

Delia, J. G., (1977). Constructivism and the study of human communication. Quarterly Journal of Speech, 63. pp. 66-83.

Elrich, M. (1994). The stereotype within. Educational Leadership, 51. pp. 1215.

Frey, L. R., Botan, C. H., Friedman, P. G. \& Kreps, G. L. (1992). Interpreting Communication Research: A Case Study Approach: Englewood Cliffs, NJ: Prentice Hall. 
Guba, E. G. (1978). Toward a Methodology of Naturalistic Inquiry in Educational Evaluation. CSE Monograph Series in Evaluation no. 8. Los Angeles: University of California, Los Angeles, Center for the Study of Evaluation, as found in Patton, M. Q. (1990). Qualitative Evaluation and Research Methods. Newbury Park, CA: Sage.

Guild, P. (1994). The culture / learning style connection. Educational Leadership, 51. pp. 16-21.

Janzen, R. (1994). Melting pot or mosaic? Educational Leadership, 51. pp. 911.

Kelly, G. A., (1955). The Psychology of Personal Constructs. New York: W. W. Norton \& Company Inc.

King, J., \& Ladson-Billings, G. (1990). The teacher education challenge in elite university settings: Developing critical perspectives for teaching in democratic and multicultural societies. European Journal of Intercultural Education, 1.pp. 15-20.

Kottak, C. P. (1982). Anthropology: The Exploration of Human Diversity. (3rd ed.) New York, NY: Random House. 
Ladson-Billings, G. (1994). What we can learn from multicultural education research. Educational Leadership, 51. pp. 22-26.

Lau v Nichols. (1974). 414 U.S. 563. as found in Nieto, S. (1992). Affirming Diversity: The sociopolitical Context of Multicultural Education. White Plains, NY: Longman Publishing Group.

Lieberman, D. A. (1994) Public Speaking in the Multicultural Environment. Englewood Cliffs, NJ: Prentice Hall.

Littlejohn, S. W. (1992). Theories of Human Communication. (4th ed.) Belmont, CA: Wadsworth.

Lofland, J., \& Lofland, L. H. (1995). Analyzing Social Settings: A Guide to Qualitative Observation and Analysis. (3rd. Ed.). Belmont, CA: Wadsworth.

Marshall, C., \& Rossman, G. B. (1989). Designing Qualitative Research. Newbury Park, CA: Sage.

Morgan, D. L. (1993). Successful Focus Groups: Advancing the State of the Art. Newbury Park, CA: Sage.

Nieto, S. (1992). Affirming Diversity: The sociopolitical Context of Multicultural Education. White Plains, NY: Longman Publishing Group. 
Paine, L. (1990). Orientation towards diversity: What do prospective teachers bring? (Researach Report 89-9.) East Lansing, MI: National Center for Research on Teacher Education.

Patton, M. Q. (1990). Qualitative Evaluation and Research Methods. Newbury Park, CA: Sage.

Porter, R. E., \& Samovar, L. A. (1994). Intercultural Communication A Reader. (7th ed.) Belmont, CA: Wadsworth.

Rosenthal, R. \& Babad, E. Y., (1985). Pygmalion in the gymnasium. Educational Leadership, Sept. pp. 36-39.

Rosenthal, R., \& Jacobson, L. (1968). Pygmalion in the classroom: Teacher expectation and pupil's intellectual development. New York: Holt, Rinehart \& Winston.

Ross, D. D., \& Smith, W. (1992). Understanding preservice teachers' perspectives on diversity. Journal of Teacher Education, 43. pp. 94103.

Rubovits, P. C., \& Maehr, M. L. (1971). Pygmalion analyzed; Toward an explanation of the Rosenthal-Jacobson findings. Journal of Personality and Social Psychology, 14. pp. 197-203. 
Rubovits, P. C., \& Maehr, M. L. (1973). Pygmalion black and white. Journal of Personality and Social Psychology, 25. pp. 21-218.

Sharp, D. L. (1922). Education in a Democracy. Cambridge, MA: The Riverside Press.

Smith, M. J. (1988). Contemporary Communication Research Methods. Belmont, CA: Wadsworth.

UNESCO. (1955). Interrelations of Cultures: Their Contribution to International nderstanding. Kleber, Paris: Buchdruckerei Winterthur AG., Winterthur.

Williamson McDiarmid, G. (1992). What to do about differences? A study of multicultural education for teacher trainees in the Los Angeles unified school district. Journal of Teacher Education, 43. pp. 83-93.

Worsnop, R. L. (1993). Bilingual education. C Q Researcher, Aug. 13. pp. 699-714. 
APPENDIX A

RESEARCH PROJECT CORRELATION / OVERLAP 


\section{RESEARCH PROJECT CORRELATION / OVERLAP}

\section{LEGEND}

Community

Mid-level Students

Diversity Cohort Advisors

Diversity Thesis Candidate

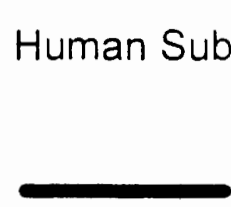

Human Sub.
Focus Grp.

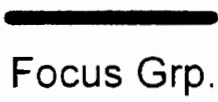

Interviews

Interviews

Program Evaluation

Survey

Human Sub.

Focus Grp.

Interviews

Human Sub. 
APPENDIX B

EMERGENT THEMES (LITERATURE REVIEW) 


\section{EMERGENT THEMES (LITERATURE REVIEW)}

\section{CONSTRUCTS:}

Ethnocentric / Ethnorelative

Assimilationism / Pluralism

Good / Bad

Active / Passive

Growing / Dying

Comfort / Threat

LOWER ORDER CONCEPTS:

Race

Socioeconomic Status

Language

Learning Style

Sexual Orientation

Occupation

Religious Affiliation

OTHER CONCEPTS:

Culture
Gender

Ability

Democracy

Teaching Style

Age

Hobbies

Difference 
APPENDIX C

SURVEY SCRIPT 
SURVEY SCRIPT

What I am about to hand you is a survey designed to collect some data regarding the Children and Communities cohort program. It is important you remember, throughout the survey, that the cohort advisors are interested in your truthful answers not what you think they want to hear. Your identity will be coded with the numbers on your survey and only I will have the list of names corresponding to those numbers. Therefore, your advisors will have no way of determining who provided which answers. This survey has no impact on your grade and is for research and program evaluation purposes only.

It is also important for you to note that throughout the survey the word "program" refers to all the classes, field trips, assignments, practicums, student teaching, and projects you have completed as a part of the Children and Communities cohort.

You may choose not to complete any item on the survey and you may stop at any time. However, the evaluation is most useful if you complete all items.

Remember, the researchers are interested in your opinions and experiences, not "correct" answers or what you think they want to hear.

Are there any questions? 


\section{APPENDIX D}

PROGRAM EVALUATION OVERVIEW 


\section{PROGRAM EVALUATION OVERVIEW}

Children and Communities Program Evaluation - Overview and analysis Oct. 31, 1995

Following is a thumbnail sketch of the items and concepts I found to be of interest. With each trend are questions which may be relevant to either a focus group or an indepth interview

\section{OVERVIEW}

Generally, the survey suggests about five major trends that hold fairly consistent across sections of questions.

First, in terms of previous social contact, nearly every question (previous contact with persons of another culture, linguistic group, socio-economic group in work, college or socially) is skewed toward the longer periods of contact. Yet when individuals are asked to indicate whether the Children and Communities program has provided no contact, increased contact or substantially increased contact with these same groups the sample is skewed toward increased and substantially increased contact for responses. This brings up two issues. Perhaps the concept of contact should be explored, again, what is considered contact and what is not? When students pictured social contact with these groups to answer the questions what was that contact like? Could the students describe previous contact? Could they describe the nature of their increased contact? Perhaps what was increased was the depth of contact or quality of contact as opposed to the amount of contact. Second, a consideration of demand and social desirability characteristics may play a role here.

Second, with regard to mid level students, respondents indicated both an increased desire to work with and understanding of this group. However, respondents also indicated their skills for working with this population had generally not changed in 11 cases or had increased in 8 cases. Only 2 individuals indicated their skills for working with mid level students had substantially increased. Questions this prompts: What implications does this have for students career choices? If they feel unqualified, will they choose this field less often? What types of skills do they feel they need? How could the program be improved to address these skills? If a student is asked to imagine him or herself in 
a mid level classroom as a teacher, what concerns will he or she have? What areas are the most salient to the students?

For most of the entering beliefs questions, students answered such that they believed cultural diversity and community were important to teaching. However, when asked about program influences most students indicated a change in these areas. The glaring questions are: How has that change come about? We have an idea what you said you believed before, how has that changed? What experiences may have shaped your entering beliefs? Your beliefs changed from what to what? What types of skills do you think are important? How has the program done in providing you those skills?

The SES data indicate students came from mostly aspiring, solidly, and upper middle class backgrounds. Not surprisingly, the students they would like to teach are those from the same types of backgrounds. Generally, students currently are at a slightly "lower" (I do not have the language to properly express my thoughts here) SES than when they were growing up. Also, the students they are currently teaching are of a slightly "lower" SES than those students they would like to teach? Questions: What implications does this have for the classroom? How does the concept of appreciating diversity fit in? What does this mean about the feasibility of teaching about the concept diversity? What does the teaching career provide in terms of an economic opportunity? Issues: People identifying with those who are similar to themselves. Teachers teaching students of different backgrounds. Teachers teaching students that are not "ideal" in the instructors mind.

Finally, the ranking of schools according to "better education" fell out as somewhat inconclusive. An equal number of respondents answered "yes" and "no" to the statement "Kids at suburban schools get a better education than kids at urban schools." Generally, respondents answered "no" to the statement "Kids at urban schools get a better education than kids at rural schools." It is unclear if respondents intended one to be better than another or that all may provide an equal opportunity for "better education." However, respondents generally indicated their perceptions about urban schools had changed somewhat. 
APPENDIXE

SURVEY THEMES 


\begin{tabular}{|c|c|c|c|c|c|c|c|c|}
\hline & & & & & & & & \\
\hline & & & $\begin{array}{l}\text { SURVEY } \\
\text { THEMES }\end{array}$ & & & & & \\
\hline & & $\begin{array}{c}\text { DIVERSITY } \\
\text { AS... }\end{array}$ & & & & & & \\
\hline & & & & & & & & \\
\hline & & & & & & & & \\
\hline & ID & difference & culture & race & SES & language & beliefs & values \\
\hline & 1 & & 1 & 1 & & 1 & & \\
\hline & 2 & 2 & 2 & 2 & 2 & & & \\
\hline & 3 & 3 & 3 & & 3 & & 3 & \\
\hline & 4 & & & & & & & \\
\hline & 5 & 5 & 5 & 5 & 5 & & & \\
\hline & 6 & 6 & 6 & 6 & & 6 & & 6 \\
\hline & 7 & & & & & & & \\
\hline & 8 & & & & & & & \\
\hline & 9 & 9 & 9 & & & & & \\
\hline & 10 & 10 & 10 & & 10 & & & \\
\hline & 11 & 11 & & & & & & \\
\hline & 12 & 12 & 12 & & 12 & & 12 & 12 \\
\hline & 13 & 13 & 13 & & & & & \\
\hline & 14 & 14 & & 14 & & & & \\
\hline & 15 & 15 & 15 & 15 & 15 & & 15 & \\
\hline & 16 & 16 & 16 & & & 16 & & \\
\hline & 17 & 17 & 17 & & 17 & & & \\
\hline & 18 & 18 & & 18 & & 18 & & \\
\hline & 19 & 19 & & & & 19 & 19 & 19 \\
\hline & 20 & & & & & & & \\
\hline & 21 & & & & & & & \\
\hline & 22 & 22 & & & & & & \\
\hline & 23 & 23 & 23 & & & & 23 & 23 \\
\hline TOTAL & & 17 & 13 & 7 & 7 & 5 & 5 & 4 \\
\hline & & & & & & & - & \\
\hline & & & & & & & & \\
\hline & & & & & & & & \\
\hline & & & & & & & & \\
\hline & & & & & & & & \\
\hline & & & & & & & & \\
\hline & & & & & & & & \\
\hline & & & & & & & & \\
\hline & & & & & & & & \\
\hline & & & & & & & & \\
\hline & & & & & & & & \\
\hline
\end{tabular}




\begin{tabular}{|c|c|c|c|c|c|c|c|c|}
\hline & & & & & & & & \\
\hline & & & & & & & & \\
\hline & & & & & & & & \\
\hline & & & & $\begin{array}{l}\text { SUR } \\
\text { THEN }\end{array}$ & $\begin{array}{l}\text { EY } \\
\text { ES }\end{array}$ & & & \\
\hline & & & & & & & & \\
\hline & & $\begin{array}{c}\text { DIVERSITY } \\
\text { AS... }\end{array}$ & & & & & & \\
\hline & & & & & & & & \\
\hline & & & & & & & & \\
\hline & ID & background & ehtnicity & customs & ability & honor & $\operatorname{sex}$ & exper. \\
\hline & 1 & & & & & 1 & & \\
\hline & 2 & & & & & & & \\
\hline & 3 & & & & 3 & & & \\
\hline & 4 & & & & & & & \\
\hline & 5 & & & & & & 5 & \\
\hline & 6 & & & $\overline{6}$ & & & & \\
\hline & 7 & & & & & & & \\
\hline & 8 & & & & & & & \\
\hline & 9 & 9 & & & & & & 9 \\
\hline & 10 & & & & & & & \\
\hline & 11 & 11 & & & & & & 11 \\
\hline & 12 & 12 & 12 & & & & & \\
\hline & 13 & & & & & & & \\
\hline & 14 & & 14 & & & & & 14 \\
\hline & 15 & & 15 & & & & & \\
\hline & 16 & & & & & & & \\
\hline & 17 & & & & & & & \\
\hline & 18 & & & & & & & \\
\hline & 19 & & & 19 & & & & \\
\hline & 20 & & & & & & & \\
\hline & 21 & & & & & & & \\
\hline & 22 & & & & & & & \\
\hline & 23 & & & 23 & & & & \\
\hline TOTAL & & 3 & 3 & 3 & 1 & 1 & $\overline{1}$ & 3 \\
\hline & & & & & & & & \\
\hline & & & & & & & & \\
\hline & & & & & & & & \\
\hline & & & & & & & & \\
\hline & & & & & & & & \\
\hline & & & & & & & & \\
\hline
\end{tabular}




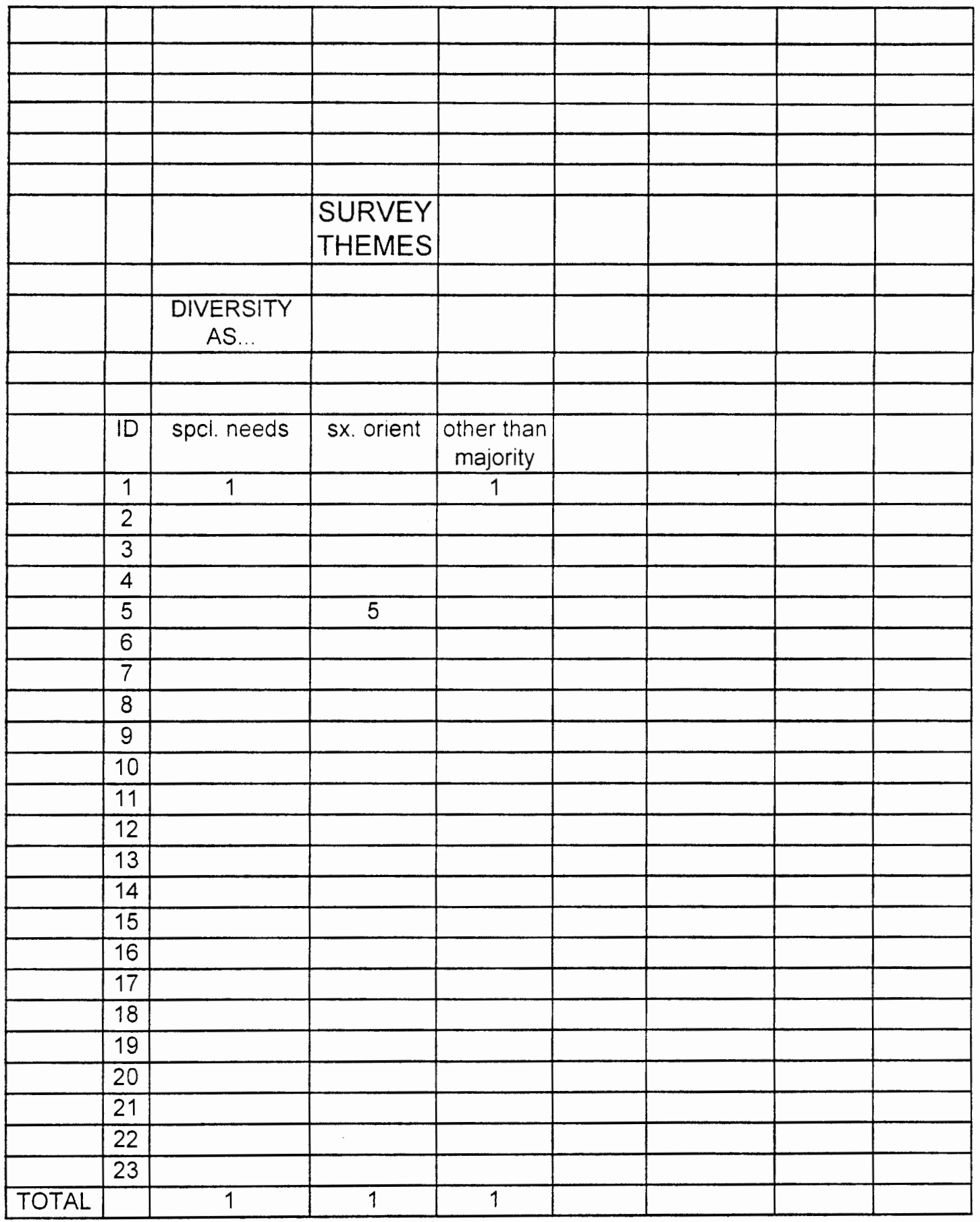


APPENDIX F

INFORMED CONSENT 


\section{Informed Consent Form}

I, agree to take part in this research project on the Children and Communities Cohort of the Graduate Teacher Education Program of the Department of Curriculum and Teaching of the School of Education at Portland State University.

I understand that the study involves my participation in a survey, in a focus group and possibly in one-on-one interviews. I further understand that participation in the focus group and in the interviews will be videotaped and audiotaped for later analysis. I understand that these tapes will only be used by the principal investigators of the study, and that if in the presentation of findings the video is used, it will be reviewed and or disguised to avoid disclosure of my identity. If that is not possible, prior to such presentational use, I will be contacted and offered an opportunity to view the material to be presented and must give my approval prior to the use of that material.

I understand that because of this study the faculty involved in researching and evaluating the program and my responses about the program will also be involved in evaluating my personal progress in the program. I understand that they have agreed to form an internal panel to review decisions which have a negative impact on my participation in the program and that a major point of this review will include a discussion about whether or not confidential research participation influenced the decision, and in the event that the panel does conclude that research study data influenced the decision, they will ask the GTEP program to convey a review of the use of the data in the evaluation considering its impact(s) on my evaluation and participation in the program.

Professors Henry, Musser, and Noordhoff and graduate research assistant Vogt have told me that the purpose of the study is to learn more about the effectiveness of the Children and Communities cohort.

I may not receive any direct benefit from the study but the study will help increase knowledge that may help the program and others in teacher education in the future.

Professors Henry, Musser, and Noordhoff and graduate research assistant Vogt have offered to answer any questions that I have about the study and what I am expected to do. They have promised that all of the information I give will be kept confidential to the extent permitted by law. and that the names of all of the people in the study will remain confidential.

I understand that I may withdraw from this study without hurting my course/program grade or relationship with Portland State University.

I have read the above information and agree to take part in this study.

Date:

Signature

If you have concerns or questions about this study, please contact the Chair of the Human Subjects Research Review Committee, Research and Sponsored Projects, 105 Neuberger Hall, Portland State University, 503/725-3417. 
APPENDIX G

CAMPUS MINISTRY LETTER 


\section{CAMPUS MINISTRY LETTER}

February 11, 1996

Administrators of Campus Christian Center:

This letter serves to convey our thanks for the use of your "K-House" facility on February 10, 1996. We were extremely pleased with the facility and its condition on the date of use and we would highly recommend its use for other group functions in the future.

In spite of the tragic floods that occurred in Portland and closed Portland State University on Thursday and Friday, February 8 and 9, preventing our representative from securing a key to open the facility Saturday morning, the "K-House" facility was open and ready for use by 7:30 am. It was clean and prepared for our group activities. We were pleased to know that someone was looking out for us in such a chaotic time. Additionally, the facility was provided to our group free of charge and we greatly appreciate such a generous gesture. We will keep your facility in mind for future events and will recommend it to others.

Thank you,

Terrine L. Brown Vogt

Graduate Research Assistant

Education Department 
APPENDIX H

FOCUS GROUP TRANSCRIPTS 
Again, one of the concepts that you've been working with is that idea of diversity, and since you've spent some time talking today, I'd like to start this section with again, some of the definitions that you might have of that concept.

Well, we just talked about a little bit of diversity in the classroom and it had to do with a speaker coming in and talking about Reparian zones and the environment, and how, if you have, um, along a stream, just, um, like, all ivy, you know you need the diversity to bring in different animals, and different birds, different foods and nutrients, and some could help filter out pollutants and some could help, um, keep the soil and ground, and then we brought that into the classroom, and why is diversity important. And so we were talking about even the male and the female, and the different grades, and then, um, just talking how everybody has something unique to offer and just the variety, and uniqueness, and, um, different resources.

That about covers it - let's go home!

Is there any one part of that that strikes you as, you know, particularly important about the definition, what (tape over, interruption) 
Everybody has their own thing, um, to offer to larger groups, everybody has their own strengths, they um, to complement each other. You know.

Our school, um, just had a really neat event called a cultural fair, um, the eighth graders, it's a school wide project that all the eighth graders had to do. And basically it's researching their family history and tying that to themselves. And all the kids - I don't know if you got a chance to see it or not.

Ya, I did.

OK - all the kids made these wonderful displays and it was amazing walking through the gyms because there was such diversity - I mean, there were people from all over the place. And it was, it was a celebration of it - no one was being put down for being this, or put down for being this. I mean it really was to me, it was just fascinating. And some of them had brought out samples of foods from, um they weren't to eat, just for show, different countries; others had picture books. I mean, you know, there were kids from Vietnam, obviously, um, Armenia, um, a, Russia, Ethiopia, um - I mean it just - It was endless - I had no idea that we had so many people from so many different places at Mt. Tabor! And, um, all the - Did your kids also get a chance to see it? 
Ya. They set it up because you know when they had the...upstairs they rearranged it so the kids...

I know we were able to see it because we have eighth graders in our class, but they did set it up to where each grade could go and visit. Ok, and, and - I just think that it was the neatest, the neatet experience and - It was awesome to see how much work the kids had put into it, and how proud they were! I thought, I mean it, it was just - Sorry

I liked the effect it had on the kids when we took them there. Because I think at first they thought "Oh we're just going to get out of class to see this.", but actually when they walked through, there were just rows and rows of all these...awards...they had clothes, food, I mean, jewelry and pictures, and everything, and it was so amazing to them because they walked in and they were like "wow!' I mean it wasn't what they were expecting, and I think it really affected them. You know, they thought, when I get into eighth grade, I know that I'm gonna do this. Or they're thinking about different things they can do when they get to the eighth grade and so it impressed them a lot more than they thought and it affected them a lot more than they imagined it would and - 
And, ah, sorry go ahead, it was also amazing because we have, um - I don't know if you guys do this or not, but we have extra projects, ok, and the eighth graders actually for their extra project did their cultural fair instead of doing another "I search" paper and so we really got to see, um, the whole process they went through, so to speak, to get to that final display piece, and part of our extra project requirements is that there's a class participation component and I remember one student had, um, she, uh, she had um, someone had come over on a boat, so she had made boat hats out of newspapers. She took two students, because it was a couple, and she took, you know, a mother and father and, she took them and made them wear the boat hats. Then someone, when they came to the states, their kids started a popcorn thing so she had, um, two little popcorn balls, so the two kids who were representing their mother and father had to come up and hold the popcorn. It just really got everybody involved, and everybody was so excited and then she shared this whole, um, she shared this food with the class that was traditional where she was from, it was just, it was the neatest experience. It really celebrated diversity. I really enjoyed that. So -

And to me too, and it sounds great, and l've been in classrooms where, you know, they just did one big celebration, but I think it should be ongoing too, and my mentor teacher has done a lot to show me about that, you know, um, whe 
just does, um, she just does all kinds of stuff. The teacher I work with starts off in the beginning of the year too - celebrated the diversity of people where it first started off with everyone has different eyes, and it showed everyone with different noses. It just went through everything. At the end, it talked about how, um, you know, everyone has different colored skin and how we're all beautiful and how different people have pets of different kinds - and it went some people have cobras in some countries for pets. And I mean it just really laid it out in a clear way so we did, um, our self-portraits from that. And she's done things like all year long here and there to celebrate and... we had a student go to Mexico for a while with family and then come back with stuff, so we talked about where he was going and what he did while he was there. We had a young child visit, she was from the Philippines. And we talked about - and so we, we tried to also make, um, it real to the kids. You - It's like this student in your class and she's from the Phillipines...It's little increments throughout the year. I've been in classrooms where they just, you know, just do one unit on it and that's it, and ok we're done with that, move on to the rest... It should be a part of the everyday curriculum.

We have some kids.... Handicapped? No, what do you call it - sensory impaired? and I realize how fortunate we are to be in a community with all these different students - I don't know. 
Um, my teacher, I was asking the teacher for materials for Black History month

Your teacher was too hugh? (laughter)

And she was saying that she was talking with the planning team and, you know, that they do lots of black history, Hispanic, you know - It's fine to do that, but if you're going to do one, you've got to do everybody, you, you know, can't just pick these few things. We need to - we don't do anything for the Asian community, you know. We don't - and some other things she said. You know, if we're going to do one we have to do everybody, you know. - So, I don't know how that...but it's the way you would do it is, uh, is include it in the classroom instead of just having different months because at the 1 st $\& 2$ nd grade level, they're egotistical...

I think also, too, is uh, learning to accept it yourself, and we're trying to teach young kids, ah, the children in your classroom to accept it, and I, I did a thing the other day, on their family tree, um, and um, we have, um, - we have an Asian child in the classroom, and, you know, I set parameters up, to include the siblings at the top, your parents and your, and you know, grandparents - I had to set parameters, or the kids would, you know - but this one child, to him and in 
the Asian Community, cousins, and aunts \& uncles are also included in there so his whole tree was covered. And my mentor teacher didn't - I mean she's like "You can't even see his tree." And I thought, well, you know, this is his family tree to him and I have to let him do that, I have to accept that, you know, he needs to go beyond my parameters because, you know - there's two, and, I don't know, 1 just thought, you're teaching it, but you're not really accepting it yourself too. Even though you set parameters, you have to be willing to be -

Flexible. Flexible. Flexibility, key word. (laughter)

Ok. Any other things about just a definition? OK, then, kind of one more step down, a little bit more concrete, if I were to ask you and it gets along back toward what you were talking about, a little bit - if I were to ask you to picture, based on your experience, since you've taught for a while now, you know, to picture what a diverse classroom would look like for you, could you talk a little bit about what you see? You're fighting to speak! (laughter)

I think I know... when you think of diversity, a lot of it is race, you the uh, but also it's more than that, it's background, it's learning abilities, it's talents, it's so much more. So I think - I don't think you cannot have diversity, um, I think it helps 
having different cultures I think but, but you just cannot have - I mean, we have diversity in our cohort - Even though a lot of us, I mean, we all want to be teachers, we're all similar ages, similar backgrounds, we all live in Portland, but there are so many different interests and resources and learning styles and abilities that you're always going to have diversity. You just have to, sometimes yu might just, you might have to find it more and bring it out, but it's there.

(Can't hear this speaker)

I think it's important, though, um, to have, you know,...you say, what does the classroom look like, and I think that --- the bookshelf, you know, have books that have characters of, you know, people that look like the kids at least that are in your classroom, and hopefully, you know, people that aren't in your classroom have books, um, with characters from different cultures and different races, and different experiences. It's really important at least, you know, so people can For instance, we have one little black boy in our class and, you know, he's hardly, well he's, he's hyper. I don't know - that's just my diagnosis, but anyway he doesn't pay attention very often, and, uh, we were talking about ... it was Martin Luther King Jr. day and he was just - and we were reading the book asking questions, he was just sitting up so interested; and it, it was just amazing to see; it was - that was - it was neat. But anyway, to talk to things that are 
important. It was amazing to see. We were just looking at each other, the teacher and I. You know. (laughter)

Did you have something that you wanted to add? You were fighting to speak in the beginning. (laughter)

Well, actually, it was very similar to what Lisa was saying, was, I think we tend to only think of diversity as race - I'll leave it at that - But that isn't hte case. She explained it so well. (laughter)

Ok.

You said something that should be in the classroom about diversity. My teacher does this really neat - she has this bulletin that says, "Spotlight's on You", and once a week a different child has to bring something from home, pictures or whatever that they bring - (laughter) it's a big deal in the classroom that, um, right now the biggest deal is they have to get there when Ms. Conan and myself are both there, you know teaching, and to share, the kids, asks questions about themselves, and oh, they talk about their parents, and their little brothers, and their dog that died, and it's just, it's wonderful they just talk about everything. 
Here's one more, maybe, cultural diverse idea. Um, I brought in a, um, another language in the classroom, but it wasn't - 'cause we were talking earlier about bringing things that you're passionate about, but this was... I am, but I, I had some responses from the kids in their journals about thinking of middle school and having to learn other languages and we talk about other cultures, and, um, one girl said, l'd brought up that I know some French or been to France or whatever, and she wrote in her journal, "Ms Gerber knows French, you know, hint, hint, hint..." (laugh) So I brought it up to the class so if, um we could maybe learn some vocabulary and they brainstormed a list of vocab that they would want to learn, and we incorporated it into our everyday, um, counts, our calendar. And it is so - You know sometimes when you're doing the calendar, and they're drawing, you know, you have to bring them in and, you know, this is math, let's do it. But when I do the little - even if it's just learning numbers - they are just on the edge of their seats, because it's something that they wanted to learn and it's something different, you know. At first, it's the giggles - oh, it's so funny, or oh, that looks weird. But now.... and they pick it up so quickly. So hopefully that can be carried through. And I want to try and maybe - And l'd want to try even asking them, because we have Russian and Spanish speakers.... 
We do that with our deaf students and, um, come to think of it I haven't done it the past two weeks, um, but, they get up and....teach a few things to the kids in the classroom, um, on how to sign certain things, and, um, which gives them the spotlight that they don't always get. Um, and not just in the classroom....it's really neat. They really get into it. it's interesting to see sign language as a language.

I thought I saw somebody over here who was trying to talk. Well, I guess at this point what it makes sense to do is to offer each of you just kind of take the floor for a minute, officially, to go around and kind of give a summative comment about what you think about the program, about either, you know, of the topics, community, diversity, whatever you'd like, just real briefly, we don't have that much time left. You know, just to kind of give each of you a chance. I know you may just want to bolt. If you want to pass you can do that, too. Want to go?

Um, well, I think it's amazing that it's been almost a year, time flies so quickly, and just how, I think, it's opened my eyes to more of what community and diversity means to me - and I need more time to think of that. (laughter)

You can have a second turn, if you want. (laughter) 
For me, through this program, um, the focus of this program, probably my definition of community and my definition of diversity has expanded a lot.

I was really excited when I read the focus of this cohort was children and communities. I could have been assigned to a different cohort. (laughter) It's me and l've really enjoyed everything we've learned. It's enhanced my ...and been a good experience.

For me, it's been a great, um, experience - Um, I think the thing I think is best is the school gives us....into really what we've learned about.. I know there was a time when I wanted to be a different community, um, my own community where I lived, and, um, requested that, um, though there were some partnerships with that, it continued to be that I'm still doing - I'm really glad that I was encouraged to stay....it helped me to grow a lot more.... just think that sense of community.... watch it develop and grow, and sometimes break apart too, has been a good experience. I think that for me has been the best thing, to be with a cohort and move along together and experience together, understand, you know, finding out that it's worth doing and finding out that just about everyone cried this week, it's not just me. (laughter) So - I guess the whole experience.... 
I think that it helped me a lot because I hadn't thought a lot about those ideas until this cohort ... and it seems really strange to me now that I hadn't, but that just tells me how much that l've learned and when we first were talking about this, I was like, we haven't done anything with that since like the first term, what are we going to talk about? But, oh, Aah, (laughter) we have! It's nice right now to be able to reflect, (laughter) to think about that and talk about it because you never have enough time to, to talk, to think about the big ideas and after we leave this, probably we won't again for a while, so....

(Can't hear speaker, but I think it's a guy)I think the cohort experience has been a great example of a community in all its definitions - a real rewarding experience. I think the professors have modeled, for some of us who don't have a lot of classroom experience, how a teacher acts, how they treat other teachers, $\ldots$

I think what l've really learned is that you can't teach in a vacuum. Kids, um, you know, I went in thinking, well, I'll teach this, this, and this... these kids are gonna learn, you know, and it's all gonna happen. But, I mean, the kids aren't blank slates; they come, you know, from what they've had, you know, all their lives up until then, and you have to - you can't just ignore the community because you're just gonna bang your head against the wall because it's not 
gonna work. And you have to really think about the, um, community in terms of the kids - you have to build the community in the classroom. Um, you know, basically, it won't work otherwise.

whatever you guys want to say is, is wonderful - whatever it is, is whatever it is. Because it's not like, it's really just understanding what it is you've come away with... The only people who will look at my thesis will be Samuel and the 2 people on my thesis committee, and ... In terms of my thesis, the research itself....

Right now this is for my master's, but I'm getting a doctorate....ok, enough about me. The other general topic for discussion is the conceptt of diversity, another one you've been dealing with sort of throughout the entire program. I'd kind of like to start on the same lines, at least parallel a little bit to the beginning, and ask you to talk a little bit about some of the definitions that you have for that concept again. (heavy sigh)

There can be economic diversity, there could be cultural diversity. Those are two main ones. 
Academic diversity?

There's diversity in preadolescents.

When I think of diversity I think of looking at different cultures and accepting teaching the kids to do that and having an activity like this is Black History month, so we incorporate some of that in the program, or it's Chinese New Year, have something going on for that, or Tet, which is new year for Vietnam. Especially in our school, Vietnamese program, we have a large Vietnamese program there so we're trying to work with some of our students doing an activity, 'cause we've got three Vietnamese students in our class they're second and third graders, so, the um, we're doing some reading, literature, opening their eyes, getting certain kids ideas who haven't seen this stuff before, or know about a certain culture, so -

In our school we have diversity not only in cultures but in, um, a, special, special ed - we have deaf, deaf kids, we have mentally, the um, mentally challenged children within the school itself, and that's quite a bit of diversity. 
The diversity I see most is the academic - it's the range and level at which kids learn, there's more than one level in the sixth grade class it's like there's such a huge span of, of what grade you're in. There's kids always at the bottom, some kids at the top and just a bunch in the middle. It's just - it kind of blew me away at first, the level at which - I kind of hoped that the level at sixth grade, that everyone would be at the same level, but it's not true. There's just such a big span.

Diversity...ways that children think and solve their problems. I don't know if this really pertains to this discussion, but about a week ago, we had been working on our subtraction and, um, I wasn't quite satisfied that I knew where every child was at. So I went ahead and gave them a test. It's just - a couple of them got done early and said "Could we draw pictures?", and I said "Sure, go ahead." And it was incredible. About two thirds of the class got to the pictures. It was so interesting! The pictures - to me I was looking at the way they felt. I mean, the subject matter shows - and the colors they used really revealed to me how they felt about that test. And it didn't necessarily reflect how they did either, um, so. But it was sort of a diversity of the way they felt at that moment. And 1 thought, I should do this more often, just, just to let me know how they're feeling and keep in touch with what they're thinking. And of course, the whole test, I 
said, you know, please show me how, what you're thinking. They did well. It was helpful.

(can't hear next conversation)...interactions... Cindy's best friends with judy and the next day Judy doesn't like Sue, and that dynamic has an effect on whether they are donig their work in the classroom. And that's been a real difficult thing in the classroom. And a lot of children need - It's very important that... I'm starting on my second sociogram...math and I hope to do a third by the time it's just fascinating who they want to sit next to or... and who's in and who's out.

If you talk about diversity being important, um and we talk about like - but teachers are put into a problem... diversity, teachers are feeling that... reality kids, kids see that that's... understand that there's mixed signals as far as diversity is concerned, you know, saying diversity is good, but yet, that's not really what's happening, diversity is not, you know, the message is being sent that it's not. It's better to be one way, it's better to be the strongest, you know, that's it. So you can say diversity is good, but it's not happening. It's not being practiced. So you're giving mixed signals - the teachers are in difficult positions as far as that's concerned...diversity's good. I think everybody agrees. We all agree that it's good, but - I mean you know, you come to the point where you've 
got to make a decision about how much time you spend with the kids that have learning problems; the resources the budget cuts and always a certain amount of students are gonna get .... diversity is a good concept, we should teach it, but it can be counterproductive in some respects if it's not, if it's not the whole of the parts, the whole thing so I think it's good to teach kids to work together, that's the most important thing to me. lit's not what they see, it's what they see is that they've got to be better than the others - um - It's a tough thing. Diversity, it's a tough thing.

In a sense, so what you're saying, is the real world doesn't practice what we preach? At all? We can teach them about diversity, and talk about diversity and use diversity in the classroom but when we get outside, it's different.

Even then - even in the school, just grades, you know, grades are based on outcomes are a good idea, but if you - in its purest form, but most schools have an evaluation where you have to rate it and kids understand it, and if they don't perform well - yeah, it's in the classroom, it's in the real world. What students see as important, something that's really there, ...you know.

I guess I can see that because when like you've got all these papers, kids, you know, with different levels of achievement and different accomplishments and 
different knowledgement. Tracy can't read, and so-and-so can read, it's like, as a teacher, what do you make of it? When you go to do the report cards like, well, Casey learned five words so, oh my gosh, you know. I'm going to give him the best grade, and then here's some other kid that, without an effort, always messing around in class, but reads a seventh grade book. You just... when it comes time to explain tao the parent, well, this kid got lower than Casey because I, I don't - It's really hard to specialize your expectations for each student. But you feel like you have to, because it's not, it's like that five words for Casey makes me want tao jump up and down and scream and holler because that other kid that's so high, it's like, I don't feel like that - it was just messing around - and it's true that all the cooperative groups and everything, it feels like it's the right thing to do, but when you get out, you're on your own. You've got to go take a standardized test, usually, to do anything you want to do, you're not gonna be able to pair up with someone and say, well, this is my strength, what's yours? (laughter) Let's put them together. It doesn't work like that. I like having kids work together, I think they learn a lot from it, but I also think that when you get out there, I have needs. That's all. I don't WANT it to be like that, I don't think it should be, but it is.

I think kids see, too... we have some modified programs for some of our kids that don't learn as fast as the other ones, so we cut down some of the - and they 
can do great on their work, on their modified work that they turn in, they can get an $\mathrm{A}$ on the work they turn in, but then the other kids remind, always remind them that they're not doing the same amount of work as they're doing, so they're grade is not really an A. It's, you know, not justified the same way theirs is, because their, their lesson or their work is cut in half.

But do you give them an A for their grade?

Ya, we cut down their workload, cut in half from what the other kids get, so even if they do their work and do it good, they get an A. But the other kids always remind those kids, 'cause those kids always seem to figure out which kids are on the program - 'cause they always find out what kids are being helped and that kind of stuff, and 'cause kids will come up to me afterwards, I'll say, good job, you're doing really good, and they'll say, Yeah, but it's not the same as, you know, Joe over here, you know, who's doing all the work. The kids always remind them - kids are cruel that way.

Kids are cheating something... but I mean they have to make a judgement on what they're doing.... 
The kids that are on the program, at first they feel like they're doing good - yeah, I"m doing a good job. But then they leave the classroom, they'll go up to the kids and say, yeah, I got an A, too, but the other kids say, well, no it's not the same, because you're workload isn't the same as ours - yours is cut in half. You're not doing the same. Your grade can't be justified with ours. You can't say you got an A like we got an A, because it's not the same.

I think, too, I've been at schools that weren't - I think that has something to do with Fowler - I mean not that it's a bad place or anything, but diversity there is not diversity. It's not differences - essentially, how I think of diversity, is there are differences. It's not a good thing... kids are the first to pick that up. I find that in my classes, too, but in other places that you know even younger kids are going, alright, that's great...you finished that whole thing....

There's some kids that are supportive; some kids, you know, say hey, that's good. They see that someone's been struggling, so their work has been cut in half. But kids don't give them that support. You know, getting that done, you're doing a good job on that; but there's always a couple kids you know that are always reminding them that they're not doing the same work.... 
Why do they have different objectives? Why is their load so much lower? Why

We've just found... well, there's a lot of kids that are in the sixth grade that have all these other problems outside the school, and so we try to lessen their workload to take some of the pressure off them. A lot of kids,....there's abuse, there's things going on in their life that we see that it makes it hard for them to work and concentrate in class, so we, you know, try to lessen their load. And um, some kids' levels just aren't as high as others'. To help us help them, we cut their work in half so they can spend a little more time with their classwork. And, I mean, for the most part it works well, but I had a couple kids come up to me and l'vs said to them you're doing a good job. And they always say "No" you know, "I'm not doing the same work as so and so." and you just try to reinforce them, you know, "That's not true. You're doing your share, and doing a good job."

It's not good if you're saying....it gets the kids thinking, I think, in this context, you're not going to have a perfect scenario because most kids are going to have their problems, you know, kids have advantages brought into school in a situation like that can be positive if the kids, um, that are getting the modified work can make the connection between, you know, "I did this work and I'm 
proud of it, although it wasn't that much, I did it, and maybe I can do as much as they're doing" AND some sensitivity on the part of the other kids, and that's the tough part. How do you tell those kids that what they're doing, you know, that these kids with their situation, ...how do you tell these kids, what these kids are going through is something that's... how do you begin to explain it in a way that doesn't cause any problems in the whole school...?

And the goal of the whole modified thing is to get them eventually up to where they're doing the work that the other kids are doing. That's, that's the goal. It takes time.

How do you do that?

We progressively give them more, you know, more of the workload, slowly over time, you know - we've got a couple kids...in my spelling class, where they have different units each week, and I've modified some of the kids down a little bit, and I have one kid that's almost up to the words the other kids are. I mean, some kids just need that, they have too many other stuff going on - too many other classes, or something like that. Maybe one or two classes where their work is cut in half, maybe they're doing the same amount of work in the other classes, but this one class they get a little help, kind of catch them up a little. 
I don't know how to let the children know where what each child...and some of them are getting it, and some of them aren't. How to say, how to let them know, I want you to do your best; I expect you to do your best, and yet, give them a break, because they are... that is something. I mean so far what we've done is we've set certain goals for certain children, and sort of like what you're saying. You know, the goals are modified, but they do have goals set, and, um, they are unfortunately, because the children talk to one another, and some of the children are very competitive, they know that ....almost as if giving up on themselves. I hear them say, "I;m just not smart enough, I'm just not, um, suchand-such". One little boy said, "Well, I'm not gonna know, because I'm an African American". He's already got it in his mind that for some reason that his behavior problems are ...he says, um, well, "The children don't like me because I'm" and he just used a racial slur. So that was just very, very - I'm trying to figure out. I - trying to help those children understand, you know, that all this society - I mean this is what we all say - we try to say that - but are we reall, really semding them the message...

You can tie that, that message back to society, because if you look at the way schools are structured, what we value is a certain standard of education that isn't - ,that's very book-oriented education, it isn't a value on other types of you, 
know, being a whole, complete person, it's about a certain educative standard and that's what we value. It's both societal and school has got that really ingrained. So, we don't value people who work with their hands, or tell wonderful stories, what we really still value is reading, writing, and math, um, some social studies, memorization is still key. Um, we can try to teach kids different ways, and vary our methods of how we approach these kids, but what it comes back is in this society and in this world, and the way it is ingrained, what we value is, good SAT scores, memorizing how to spell things, a good college education, um, and a good, money-making job. And the way you get those is, by succeeding in, you know, school and getting those good SAT scores, and I think that we can preach diversity, and preach that we value all people for what they are, but when it comes back to and what it all boils back down to is that as a society we do not reflect those goals.

And the standards, too...you're compared to everyone else, there's no focus on, "You can do this and this is what you do, and that's ok". There's no....you're compared with, "in seventh grade, you're supposed to know this". They come out with those books now that say, "What your first grader is supposed to know". And really, what you know in first grade is ok, and what you accomplish in first grade is great for you, and that's fine. But we have this standard of.... 
You need to know this by first grade if you're going to college - if you're going to MIT - (laughter)

\section{Exactly!}

If I could do away with anything in schools, it would be those standardized tests. Because, I mean, Sunnyside has absolutely gone ape because they scored lowest on the reading tests, right? Sunnyside had the lowest score on language arts and reading. So what do we do? We spend the majority of the day on language arts and reading, and the hell with math, social studies, or science, or anything else. That's what we have to do to bring up those scores. It's affecting the kids. It's affecting me.

We're doing the same thing; our math scores at our school were low, and so they're basically telling us they want us to teach the kids to the test. They want us to teach them the stuff that's gonna be on the test so they can bump those scores up. And that just goes against everything...

Ya, the heck with all the diversity and all the levels, you have one level - to get the school on a high level for that test. That's all that counts. 
I think...some of these kids are learning stuff at an earlier age than I remember learning any of it - likes times tables, some of these, this one girl is in third grade, she's got to her, she's up to her 9's in math class testing - 2's, 3's, 4's, 5's, 6's, 7's, up to her nines. I can't remember doing that in third grade, I think it was fourth grade, or something. It seems like kids are learning stuff at an earlier age, and I don't think we're .... what I was trying to do was compare what kids know now to what we used to know at a certain age, but that's not how it is any more. They're comparing what kids know to somebody else in Europe or in Asia - how well are we doing in the world, and that's what they think. We're low, America's low compared to other nations. We're not number one, so we have to be number one. In order to be number one, we have to compete against these other nations. Math, we're way behind, reading, - whatever it is. But definitely math.

As far as the big picture is, I really respect what both of you are saying unfortunately, I really don't see society as a whole or America as a country really changing their opinion about - Uou just turn to one factor - that is the population's just going to explode more than it is now. There's so many more people looking for jobs, trying to scratch out a living, feed themselves, I don't see that society is going to let up and say, ok, let's accept people's diversity, let's, let's cooperate with one another. I think what I'm trying to do is help these 
children, I want them to be able to be successful, be able to get a job. If we don't push them, to meet these standards then where are they? Do we really help them? I mean that's, go ahead and ....I don't see society changing its opinion about competitive standards.

I appreciate all your comments, and we're just about out of time, so what l'd like to do for the very end is to offer each of you a moment or two to give sort of a summative comment, whether you want to talk about the midlevel discussion or the community discussion, just generally about your experience in the cohort.... it's up to you....

I don't know about everybody else, but I think this last four weeks have been living hell in the classroom; the weather, the kids, - (laughter) yes, you're laughing with me - I know Chris has shared it, but, I tell you, .....that's it. That's all.

Do we have to go in order?

No, you don't have to go in order. I just want everybody to have their chance. 
I enjoyed it, my whole experience this whole last year. At Sunnyside School, I wanted to be there because of what was going on there and all that stuff, and I'm learning every day, that's all I can say. It's been interesting. Some of the kids - one thing I don't like, is, and I've done it a couple times, is get into the power thing. Come on Johnny, we have to go, ok? You have five seconds. You can walk down there, or ....the teacher's going, just grab him, don't try to reason with him first, you know, she's already done this. So you just pick him up, take him down to the office, screaming and kicking, ugh, part of the job I definitely don't like.

Back to tying diversity... and what Chris said, and the mixed messaes kids get when we try to value each of them in our lesson plans and individual, they have wants and needs, what they accomplished, when one goes from here to here and the other goes from here to here. But we don't even value their own solidarity, I am not moving my butt out of this spot, and you can't make me do it. You know, there's just, there's just that clash...I find myself focusing more on trying to find value with people around me and treating people and value my garbage man as much as I value my doctor, and trying to kind of think about kindness, and as we get more populated, kindness is going to be much more of an issue, and that's the direction that I'm trying to take, um, myself and my kids - 
kindness is essential to the way that we're going to live; bringing that value back into diversity and how we really treat people ....

I was so worried about meeting the curriculum guidelines, and setting these goals, generally that applied to everyone in my classroom, and I think now, my job is to teach these kids as much as I can about themselves and about treating other people the way that other people deserve to be treated, and, you know, increasing their self-esteem; and, you know, making learning important, to simply --- themselves more than, you know, you need to know these facts; that kinds of thing....

I would like to add an addendum to my "all finished." Um, I think this has been for myself one of the greatest experiences l've ever had. I would not have traded it for, um, I'm addicted to it - I'm addicted to the kids, I'm addicted to the school, I'm addicted to the life now. It's wonderful. It's challenging, but it's wonderful.

...You go through ups and downs like everybody else, and, but you remember when - you have to keep your focus on what our goal is here, um, our role is to do what we can for the best of the kids... You just have to keep tring to, trying to figure out what's the best thing for the kids.... 
APPENDIX I

FOCUS GROUP THEMES 
FOCUS GROUP THEMES

(Numbers correlate with transcript line numbers)

\begin{tabular}{|c|c|c|}
\hline ALL THEMES & & \\
\hline & & \\
\hline difference & taught & cooperation/competition \\
\hline 12.13 & 5.13 & 21 \\
\hline 34.37 & 23.72 & 323 \\
\hline 78.81 & 58 & 332.334 \\
\hline 93 & 74.77 & 341.345 \\
\hline 133 & 88.91 & 348.354 \\
\hline 135.137 & 104.106 & 356.363 \\
\hline 145.147 & 109.111 & 400.402 \\
\hline 165.166 & 118.119 & 438 \\
\hline 184 & 175.181 & 462.464 \\
\hline 269 & 274.277 & 474.479 \\
\hline 283.287 & 329 & 494.499 \\
\hline 382.385 & & 503.506 \\
\hline 486 & & 509.511 \\
\hline & & 11.13 \\
\hline
\end{tabular}




\begin{tabular}{|c|c|c|}
\hline ALL THEMES & & \\
\hline mixed signals & experiences & everyone \\
\hline 112.118 & 21.54 & 11.12 \\
\hline 131.132 & 84.88 & 20.21 \\
\hline 158.159 & 71 & 100.103 \\
\hline 216.219 & 96 & 104 \\
\hline 285.288 & 113.118 & \\
\hline 312.326 & & artifacts \\
\hline 328.331 & preached & 34.35 \\
\hline 332.336 & 328 & 50 \\
\hline 341.354 & 458 & 84.85 \\
\hline \multicolumn{3}{|l|}{383.385} \\
\hline 402.406 & language & practiced/done \\
\hline 410.421 & 172.187 & 74.75 \\
\hline 424 & 189.193 & 312.317 \\
\hline \multicolumn{3}{|l|}{427} \\
\hline 442.445 & rhetoric & accepting it \\
\hline 447.461 & 32 & 109 \\
\hline 462.467 & 75.77 & 116.119 \\
\hline 474.479 & 84 & 505.506 \\
\hline \multicolumn{3}{|l|}{486.487} \\
\hline 501.511 & ethnicity & see through it \\
\hline \multirow[t]{2}{*}{172.177} & 35.37 & 383.385 \\
\hline & 101.103 & 439.445 \\
\hline different functions & 274 & \\
\hline 8 & & teaching/learning style \\
\hline 12 & ongoing & 320 \\
\hline \multirow[t]{2}{*}{350.352} & 75 & 347.354 \\
\hline & 88.91 & 356.363 \\
\hline standards & 104.105 & 398.401 \\
\hline 112.119 & 169.171 & 402.406 \\
\hline 341.348 & 177.181 & 432.437 \\
\hline 462 & 182.187 & 447.461 \\
\hline 474.479 & 271.272 & \\
\hline \multicolumn{3}{|l|}{486.487} \\
\hline \multicolumn{3}{|l|}{507.508} \\
\hline & & \\
\hline
\end{tabular}




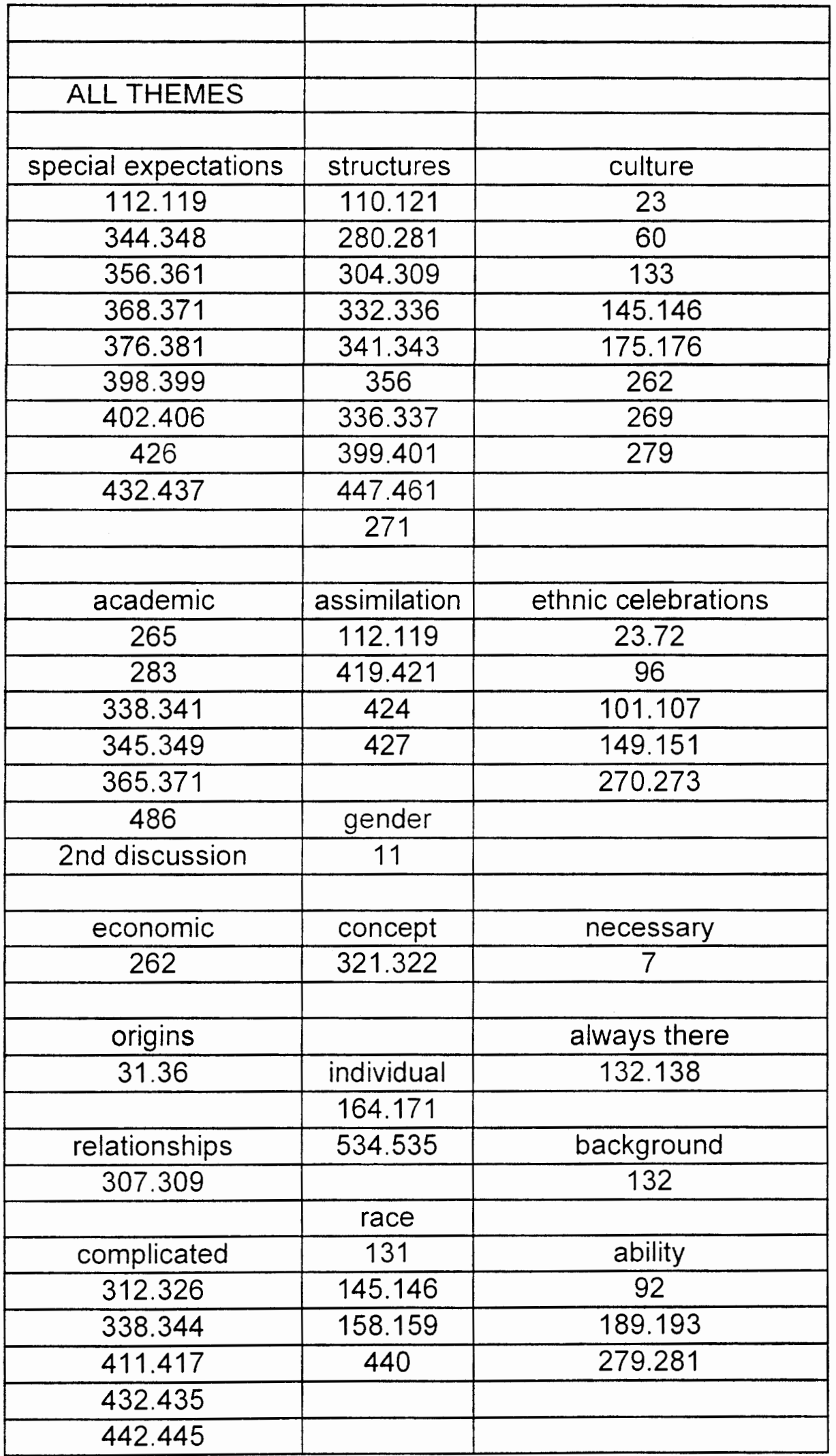




\begin{tabular}{|c|l|l|}
\hline & & \\
\hline & & \\
\hline & & \\
\hline & & \\
\hline & & \\
\hline ALL THEMES & & \\
\hline & & \\
\hline 23.52 & & \\
\hline 81.82 & & \\
\hline 84.88 & & \\
\hline 103.104 & & \\
\hline 187.193 & & \\
\hline 164.171 & & \\
\hline 172.187 & & \\
\hline 142.147 & & \\
\hline 111.115 & & \\
\hline 272.277 & & \\
\hline & & \\
\hline talents & & \\
\hline 132 & & \\
\hline & & \\
\hline good/bad & & \\
\hline 93 & & \\
\hline 312.319 & & \\
\hline & & \\
\hline & & \\
\hline
\end{tabular}




\begin{tabular}{|c|c|c|}
\hline STRUCTURES & & \\
\hline standards & structures & teaching/learning style \\
\hline 112.119 & 110.121 & 320 \\
\hline 341.348 & 280.281 & 347.354 \\
\hline 462 & 304.309 & 356.363 \\
\hline 474.479 & 332.336 & 398.401 \\
\hline 486.487 & 341.343 & 402.406 \\
\hline \multirow[t]{20}{*}{507.508} & 356 & 432.437 \\
\hline & 336.337 & 447.461 \\
\hline & 399.401 & \\
\hline & 447.461 & \\
\hline & 271 & cooperation/competition \\
\hline & & 21 \\
\hline & & 323 \\
\hline & & 332.334 \\
\hline & & 341.345 \\
\hline & & 348.354 \\
\hline & & 356.363 \\
\hline & & 400.402 \\
\hline & & 438 \\
\hline & & 462.464 \\
\hline & & 474.479 \\
\hline & & 494.499 \\
\hline & & 503.506 \\
\hline & & 509.511 \\
\hline & & 11.13 \\
\hline & & \\
\hline
\end{tabular}




\begin{tabular}{|c|c|c|}
\hline $\begin{array}{c}\text { TYPES OF } \\
\text { DIVERSITY / } \\
\text { CRITERIA } \\
\end{array}$ & & \\
\hline special expectations & difference & race \\
\hline 112.119 & 12.13 & 131 \\
\hline 344.348 & 34.37 & 145.146 \\
\hline 356.361 & 78.81 & 158.159 \\
\hline 368.371 & 93 & 440 \\
\hline 376.381 & 133 & \\
\hline 398.399 & 135.137 & ethnicity \\
\hline 402.406 & 145.147 & 35.37 \\
\hline 426 & 165.166 & 101.103 \\
\hline \multirow[t]{2}{*}{432.437} & 184 & 274 \\
\hline & 269 & \\
\hline artifacts & 283.287 & academic \\
\hline 33 & 382.385 & 265 \\
\hline 47 & 486 & 283 \\
\hline \multirow[t]{3}{*}{77.78} & & 338.341 \\
\hline & ethnicity & 345.349 \\
\hline & 35.37 & 365.371 \\
\hline language & 101.103 & 486 \\
\hline 172.187 & 274 & 2nd discussion \\
\hline \multicolumn{3}{|l|}{189.193} \\
\hline & culture & gender \\
\hline & 23 & 11 \\
\hline & 60 & \\
\hline & 133 & economic \\
\hline & 145.146 & 262 \\
\hline & 175.176 & \\
\hline & 262 & origins \\
\hline & 269 & 30.34 \\
\hline & 279 & \\
\hline & & ability/physical mental \\
\hline & & 92 \\
\hline & & 189.193 \\
\hline & & 279.281 \\
\hline & & \\
\hline
\end{tabular}




\begin{tabular}{|c|c|c|}
\hline TEACHABLE & & \\
\hline ethnic celebrations & taught & experiences \\
\hline 23.72 & 5.13 & 21.54 \\
\hline 96 & 23.72 & 84.88 \\
\hline 101.107 & 58 & 71 \\
\hline 149.151 & 74.77 & 96 \\
\hline \multirow[t]{16}{*}{270.273} & 88.91 & 113.118 \\
\hline & 104.106 & \\
\hline & 109.111 & \\
\hline & 118.119 & community based/exotic \\
\hline & 175.181 & 37 \\
\hline & 274.277 & 23.52 \\
\hline & 329 & 81.82 \\
\hline & & 84.88 \\
\hline & & 103.104 \\
\hline & & 187.193 \\
\hline & & 164.171 \\
\hline & & 172.187 \\
\hline & & 142.147 \\
\hline & & 111.115 \\
\hline & & 272.277 \\
\hline & & \\
\hline
\end{tabular}




\begin{tabular}{|c|c|c|}
\hline MIXED MESSAGES & & \\
\hline & & mixed signals \\
\hline complicated & & 112.118 \\
\hline 312.326 & & 131.132 \\
\hline 338.344 & & 158.159 \\
\hline 411.417 & & 216.219 \\
\hline 432.435 & & 285.288 \\
\hline 442.445 & & 312.326 \\
\hline & & 328.331 \\
\hline & & 332.336 \\
\hline & & 341.354 \\
\hline & & 383.385 \\
\hline & & 402.406 \\
\hline & & 410.421 \\
\hline & & 424 \\
\hline & & 427 \\
\hline & & 442.445 \\
\hline & & 447.461 \\
\hline & & 462.467 \\
\hline & & 474.479 \\
\hline & & 486.487 \\
\hline & & 501.511 \\
\hline & & 172.177 \\
\hline & & \\
\hline
\end{tabular}


APPENDIX J

INTERVIEW THEMES 
INTERVIEW THEMES

All themes:

\begin{tabular}{|c|c|c|}
\hline mixed messages & experiences & Teaching/learning \\
\hline structures & difference & why teach diversity \\
\hline values & culture & uncertain / don't know \\
\hline complicated & ongoing & teach with community \\
\hline language & listing & race \\
\hline equity & changed & critical \\
\hline standards & not prejudice & no one way to teach \\
\hline cooperation & competition & good / bad \\
\hline economic & no one definition & good to hear others \\
\hline race not an issue & always there & can't teach it \\
\hline tolerance / appreciation & political & see through it \\
\hline skills & eir ow & definition \\
\hline
\end{tabular}


Emergent Thematic Categories

Mixed Messages

mixed messages

complicated

Structures

political

competition

structures

teach with community

Types Of Diversity Or Criteria

difference

culture

economic

race

not prejudice

Teachable

experiences

ongoing

teach with community

New themes

uncertain / don't know

changed cooperation

ongoing

teaching / learning style

experiences

listing

political

language

teaching / learning style

no one way to teach

critical

no one definition

everyone their own definition 